\title{
BRAIN EDEMA AND INTRACRANIAL PRESSURE INCREASE IN STROKE: EXPERT OPINION FROM
}

\section{TURKISH CEREBROVASCULAR DISEASES SOCIETY}

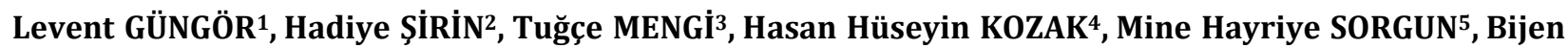
NAZLIEL ${ }^{6}$, Burcu Acar ÇíNLETi ${ }^{7}$, Şerefnur ÖZTÜRK ${ }^{8}$, Mehmet Yasir PEKTEZEL ${ }^{9}$, DILEK NECIOGLU ÖRKEN10, Mehmet Uğur ÇEVİK11, Zehra UYSAL KOCABAŞ², Ezgi SEZER ERYILDIZ13, Vesile ÖZTÜRK ${ }^{14}$, Canan TOGAY IŞIKAY ${ }^{5}$, Mehmet Akif TOPÇUOĞLU ${ }^{9}$, Bilgehan Atılgan ACAR ${ }^{15}$, Erdem YAKA $^{14}$, Aysel MílANLIOĞLU ${ }^{16}$, Derya TATLISULUOĞLU ${ }^{17}$, Nazan YAMAN ${ }^{18}$, Ayşe BİNGÖL ${ }^{5}$, Müge YEMIŞCI ÖZKAN ${ }^{9}$, Gökçe GÜRLER ${ }^{9}$, Nilüfer YEŞiLOT ${ }^{19}$, Fatma Birsen İNCE20, Leyla DöșLÜ21, Yasemin

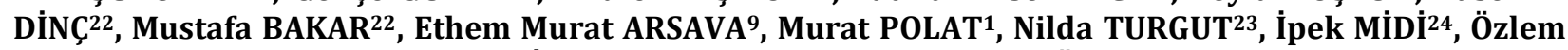
AYKAÇ ${ }^{25}$, Ufuk CAN ${ }^{26}$, Turgay DEMİR ${ }^{27}$, Şebnem BIÇAKÇI ${ }^{27}$, Mustafa GÖKÇE ${ }^{28}$, Nazire AFŞAR ${ }^{29}$, Vedat Ali YÜREKLi ${ }^{30}$, Kürşad KUTLUK ${ }^{14}$, Ali ÜNAL ${ }^{31}$, Ülgen YALAZ TEKAN ${ }^{32}$, Zülfikar ARLIER ${ }^{33}$, Dilaver KAYA ${ }^{29}$, Ayşe GÜLER ${ }^{2}$, Hale Zeynep BATUR ÇAĞLAYAN 6 , Özlem KAYIM YILDIZ ${ }^{34}$, Recep BAYDEMİR ${ }^{35}$, Hamza GÜLTEKİN36, Babür DORA ${ }^{31}$, Anıl TANBUROĞLU ${ }^{37}$, Ayça ÖZKUL ${ }^{38}$, Yasemin GÜRSOY ÖZDEMİR39, Arda YILMAZ $^{40}$, Belgin KOÇER ${ }^{2}$, Baki DOĞAN ${ }^{1}$, Erdem GÜRKAŞ ${ }^{41}$, Atilla Özcan ÖZDEMİR ${ }^{25}$, Demet Funda BAŞ$^{42}$, Semih GIRAY ${ }^{43}$,

${ }^{1}$ Ondokuz Mayıs University Faculty of Medicine, Department of Neurology, Samsun, TURKEY, ${ }^{2}$ Ege University Faculty of Medicine, Department of Neurology, İzmir, TURKEY, ${ }^{3}$ Niğde Training and Research Hospital, Intensive Care Unit, Niğde, TURKEY, ${ }^{4}$ Necmettin Erbakan University Meram Medical Faculty, Department of Neurology, Konya, TURKEY, ${ }^{5}$ Ankara University Faculty of Medicine, Department of Neurology, Ankara, TURKEY, ${ }^{6}$ Gazi University Faculty of Medicine, Department of Neurology, Ankara, TURKEY, ${ }^{7}$ University of Helath Sciences Dr. Suat Seren Chest Diseases and Chest Surgery Training and Research Hospital, Intensive Care Unit, İzmir, TURKEY, ${ }^{8}$ Selçuk University Faculty of Medicine, Department of Neurology, Konya, TURKEY, ${ }^{9}$ Hacettepe University Faculty of Medicine Department of Neurology, Ankara, TURKEY, ${ }^{10}$ Memorial Şişli Hospital Neurology Clinic, İstanbul, TURKEY, ${ }^{11}$ Dicle University Faculty of Medicine Department of Neurology, Diyarbakir

\footnotetext{
Address for Correspondence: Prof. Levent Güngör, MD. Ondokuz Mayıs University Faculty of Medicine, Department of Neurology, Samsun. Phone: +90362 3121919 Received: 02.07.2021 E-mail: ligungor@omu.edu.tr

ORCID IDs: Levent Güngör 0000-0002-3016-2137, Hadiye Sirin 0000-0003-0262-3706, Tuçe Mengi 0000-0002-0639-0957, Hasan Hüseyin Kozak 00000001-6904-8545, Mine Hayriye Sorgun 0000-0003-2370-7319, Bijen Nazlıel 0000-0002-6148-3814, Burcu Acar Çinleti 0000-0003-3369-6617, Şerefnur Öztürk 0000-0001-8986-155X, Mehmet Yasir Pektezel 0000-0003-1619-2759, Dilek Necioğlu Örken 0000-0002-1118-5309, Mehmet Uğur Çevik 00000003-0861-8588, Zehra Uysal Kocabaș 0000-0002-1838-9988, Ezgi Sezer Eryıldız 0000-0002-9653-3009, Vesile Öztürk 0000-0002-9784-6779, Canan Togay Isskay 0000-0001-6256-9487, Mehmet Akif Topçuoğlu 0000-0002-7267-1431, Bilgehan Atılgan Acar 0000-0003-2001-914X, Erdem Yaka 0000-00026644-4240, Aysel Milanlığlu 0000-0003-2298-9596, Derya Tatlısuluoğlu 0000-0002-4493-4353, Nazan Yaman 0000-0002-4257-9888, Ayşe Bingöl 00000001-8011-6641, Müge Yemişci Özkan 0000-0002-1528-1998, Gökçe Gürler 0000-0003-3073-4438, Nilüfer Yeşilot 0000-0002-9655-9487, Fatma Birsen İnce 0000-0002-6446-4897, Leyla Döșlü 0000-0001-9169-9942, Yasemin Dinç 0000-0003-0342-5939, Mustafa Bakar 0000-0001-5229-0001, Ethem Murat Arsava 0000-0002-6527-4139, Murat Polat 0000-0001-9737-8162, Nilda Turgut 0000-0001-9549-1196, İpek Midi 0000-0002-5125-3708, Özlem Aykaç 0000-0003-4987-0050, Ufuk Can 0000-0001-8689-417X, Turgay Demir 0000-0002-7076-8571, Şebnem Bıçakçı 0000-0002-0700-5088, Mustafa Gökçe 0000-0003-2965-8482, Nazire Afşar 0000-0001-8123-8560, Vedat Ali Yürekli 0000-0002-2042-4463, Kürşad Kutluk 0000-0003-4807-0289, Ali Ünal 00000002-6530-9071, Ülgen Yalaz Tekan 0000-0002-3448-7828, Zülfikar Arlıer 0000-0003-2645-648X, Dilaver Kaya 0000-0002-7364-171X, Ayșe Güler 00000003-4465-3743, Hale Zeynep Batur Çağlayan 0000-0002-3279-1842, Özlem Kayım Yıldız 0000-0002-0382-9135, Recep Baydemir 0000-0001-9753-8461, Hamza Gültekin 0000-0001-9394-4999, Babür Dora 0000-0001-5398-5905, Anıl Tanburoğlu 0000-0001-9627-3502, Ayça Özkul 0000-0001-7178-1404, Yasemin Gürsoy Özdemir 0000-0002-0860-8964, Arda Yllmaz 0000-0002-4679-1632, Belgin Koçer 0000-0003-2580-9650, Baki Doğan 0000-0003-25269279, Erdem Gürkaș 0000-0001-8086-2900, Atilla Özcan Özdemir 0000-0002-9864-6904, Demet Funda Baș 0000-0001-9231-0337, Semih Giray 00000002-0722-3181.

This article should be cited as following: Güngör L, Şirin H, Mengi T, Kozak HH, Sorgun HM, Nazlıel B, Acar Çinleti B, Öztürk Ş, Pektezel MY, Necioğlu Örken D, Cevik MU, Uysal Kocabaş Z, Sezer Eryıldız E, Öztürk V, Togay Ișıkay C, Topçuoğlu MA, Acar BA, Yaka E, Milanlığlu A, Tatlısuluoğlu D, Yaman N, Bingöl A, Yemişci Özkan M, Gürler G, Yeşilot N, İnce FB, Döşlü L, Dinç Y, Bakar M, Arsava EM, Polat M, Turgut N, Midi İ, Aykaç Ö, Can U, Demir T, Bıçakçı Ş, Gökçe M, Afşar N, Yürekli VA, Kutluk K, Ünal A, Yalaz Tekan Ü, Arlıer Z, Kaya D, Güler A, Batur Çağlayan HZ, Kayım Yıldız Ö, Baydemir R, Gültekin H, Dora B, Tanburoğlu A, Özkul A, Gürsoy Özdemir Y, Yılmaz A, Koçer B, Doğan B, Gürkaş E, Özdemir AÖ, Baş DF, Giray S. Brain edema and intracranial pressure increase in stroke: Expert opinion from Turkish Cerebrovascular Diseases Society. Turkish Journal of Cerebrovascular Diseases 2021; 27(2): 65-132. doi: $10.5505 /$ tbdhd.2021.76376
} 
TURKEY, ${ }^{12}$ Bingöl State Hospital, Intensive Care Unit, Bingöl, TURKEY, ${ }^{13}$ Manisa City Hospital, Intensive Care Unit, Manisa, TURKEY, ${ }^{14}$ Dokuz Eylül University Faculty of Medicine, Department of Neurology, İzmir, TURKEY, ${ }^{15}$ Sakarya University Faculty of Medicine, Department of Neurology, Sakarya, TURKEY, 16Van Yüzüncü Yll University Faculty of Medicine, Department of Neurology, Van, TURKEY, ${ }^{17}$ Başakşehir Çam and Sakura City Hospital, Intensive Care Unit, İstanbul, TURKEY, ${ }^{18}$ Karabük Training and Research Hospital, Intensive Care Unit, Zonguldak, TURKEY, 19İstanbul University İstanbul Medical Faculty, Department of Neurology, İstanbul, TURKEY, ${ }^{20}$ İstanbul University Cerrahpaşa Medical Faculty, Department of Neurology, İstanbul, TURKEY, ${ }^{21}$ Aydın State Hsopital, Intensive Care Unit, Aydin, TURKEY, ${ }^{22}$ Uludağ University Faculty of Medicine, Department of Neurology, Bursa, TURKEY, ${ }^{23}$ Namık Kemal University Faculty of Medicine, Department of Neurology, Tekirdağ, TURKEY, ${ }^{24}$ Marmara University Faculty of Medicine, Department of Neurology, İstanbul, TURKEY, ${ }^{25}$ Eskişsehir Osmangazi University Faculty of Medicine, Department of Neurology, Eskişehir, TURKEY, ${ }^{26}$ Başkent University Faculty of Medicine, Department of Neurology, Ankara, TURKEY, ${ }^{27}$ Çukurova University Faculty of Medicine, Department of Neurology, Adana, TURKEY, ${ }^{28}$ Kahramanmaraș Sütçü İmam University Faculty of Medicine, Department of Neurology, Kahramanmaraş, TURKEY, ${ }^{29}$ Acıbadem MAA University Faculty of Medicine, Department of Neurology, İstanbul, TURKEY, ${ }^{30}$ Süleyman Demirel University Faculty of Medicine, Department of Neurology, Isparta, TURKEY, ${ }^{31}$ Akdeniz University Faculty of Medicine, Department of Neurology, Antalya, TURKEY, ${ }^{32}$ Şișli Hamidiye Etfal Training and Research Hospital, Neurology Clinic, İstanbul, TURKEY, ${ }^{33}$ Adana City Training and Research Hospital, Neurology Clinic, Adana, TURKEY, ${ }^{34}$ Cumhuriyet University Faculty of Medicine, Department of Neurology, Sivas, TURKEY, ${ }^{35}$ Erciyes University Faculty of Medicine, Department of Neurology, Kayseri, TURKEY, ${ }^{36}$ Şırnak State Hospital, Intensive Care Unit, Şirnak, TURKEY, ${ }^{37}$ Başkent University Adana Dr. Turgut Noyan Application and Research Centre, Neurology Clinic, Adana, TURKEY, ${ }^{38}$ Adnan Menderes University Faculty of Medicine, Department of Neurology, Aydin, TURKEY, ${ }^{39}$ Koç University Faculty of Medicine, Department of Neurology, İstanbul, TURKEY, ${ }^{40}$ Mersin University Faculty of Medicine, Department of Neurology, Mersin, TURKEY, ${ }^{41}$ Lütfü Kırdar Training and Research Hospital, Neurology Clinic, İstanbul, TURKEY, ${ }^{42}$ Tepecik Training and Research Hospital Neurology Clinic, İzmir, TURKEY, ${ }^{43}$ Gaziantep University Faculty of Medicine, Department of Neurology, Gaziantep, TURKEY.

\section{ABSTRACT}

Brain edema is a common problem after stroke. Elevation of intracranial pressure causes high mortality anad morbidity rates by impairing cerebral perfusion and causing cerebral herniation. The neurologist who take care of acute stroke patients should guess the possibility of brain edema, take precautions for the development of brain edema, diagnose intracranial pressure elevation and cerebral herniation both clinically and raidologically, and treat brain edema rapidly. This expert opinion is a consensus declaration of 60 Neurologists who work on cerebrovascular diseases and neurocritical care under Turkish Society of Cerebrovascular Diseases.

Keywords: Ischemic stroke, hemorrhagic stroke, brain edema, intracranial pressure elevation syndrome, cerebral herniation, antiedema treatment.

\section{INMEDE BEYIN ÖDEMI VE KAFA İ̧̧i BASINÇ ARTIŞI: TÜRK BEYIN DAMAR HASTALIKLARI DERNEĞI UZMAN GÖRÜșÜ}

öz

Beyin ödemi inmeden sonra sık karşılaşılan bir sorundur. İntrakranial basınç artışı serebral perfüzyonu bozarak ve serebral herniasyona yol açarak mortalite ve morbiditeyi artırır. İnme hastası takip eden nörologların, hangi inmeden sonra beyin ödemi gelişeceğini kestirebilmesi, beyin ödemi gelişmemesi için önlemler alabilmesi, gelişirse intrakranial basınç artışı ve serebral herniasyonu klinik ve radyolojik olarak tanıyabilmesi, ve önlenemezse beyin ödemi ve intrakranial basınç artışını hızlı ve etkin şekilde tedavi edebilmesi gerekir. Bu uzman görüşü Türk Beyin Damar Hastalıkları Derneği bünyesinde aktif olarak çalışan 60 uzmanın ortak görüșü ile hazırlanmış bir klinik rehber niteliğindedir.

Anahtar Sözcükler: İskemik inme, hemorajik inme, beyin ödemi, kafa içi basınç artışı sendromu, serebral herniasyon, antiödem tedavi. 


\subsection{Introduction}

Brain edema can develop in various medical conditions such as head trauma, brain tumors, diabetic ketoacidosis, acute liver failure, climbing to high altitude, salicylate intoxication, cerebral hypoxia. Besides reducing survival, the development of edema in the brain after stroke also increases the rate of complications and disability in surviving patients. In this section of the Turkish Journal of Cerebrovascular Diseases, the physiopathology of brain edema developing after an ischemic and hemorrhagic stroke, the clinical syndrome and its symptoms, the laboratory methods to be used for its diagnosis and follow-up, and its treatment are explained in detail. This review has been prepared by 59 academicians and Neurology Specialists who continue their academic and daily practice on stroke and Neurology Intensive Care in Turkey. Each author compiled the section they were assigned to with the most up-to-date international literature, adding their own clinical experience and prepared recommendations that could be efficient in our country's conditions. After the preparation of the whole manuscript, all sections went through a revision process following the opinions and suggestions of all authors, ensuring that the compilation was a consensus text approved by all authors. We anticipate that it will be beneficial for all Neurology Specialists who follow acute stroke patients while providing health services in different hospitals in Turkey and for Neurology Specialization Students who continue their education.

\subsection{Brain Edema, Raised ICP Syndrome and Herniation in Stroke: Definitions and Epidemiology}

The intracranial volume is composed of $80 \%$ brain parenchyma, 10\% cerebrospinal fluid (CSF), and $10 \%$ blood in the cerebral circulation $(2 / 3$ is venous, $1 / 3$ is arterial) (1). According to the Monro-Kellie hypothesis, in case of an increase in volume in one of these compartments, this situation can be compensated to a certain extent by the shrinkage in the other compartments (Figure 1-a). In cases where the intracranial volume increases by $50-100 \mathrm{~mL}$ and the intracranial pressure rise above $15 \mathrm{mmHg}$, raised intracranial pressure syndrome (rICP) occurs (2).

Although the elasticity is low in adults, the relationship between pressure and intracranial volume may change. When there is an increase in volume in the brain parenchyma, the absorption of CSF from the arachnoid villi increases, CSF synthesis decreases, and CSF is pushed out of the cranium from the subarachnoid and ventricular area into the spinal compartment, thus reducing its volume. When the volume of the brain parenchyma increases due to edema, the intracranial blood volume also decreases as a result of increased venous drainage, the collapse of dural sinuses and veins, and narrowing of cerebral arterioles. Arterial blood gases, especially $\mathrm{CO}_{2}$ pressure, have a significant effect on the expansion and contraction of cerebral arterioles. The increased volume of the brain can be tolerated up to a certain level; this capacity is defined as compliance (1). As the intracranial pressure increases, the compliance gradually decreases, and when it rises above a certain limit herniation syndromes develop (Figure 1-b). Many factors, especially cerebral atrophy, can affect this process.

Normally, there is no pressure difference between the cavities in the head. Lesions that cause an increase in supratentorial pressure often develop subfalcine and transtentorial herniation, while tonsillar herniation develops in infratentorial lesions. Brain edema and intracranial hemorrhages after ischemia are one of the most common causes of increased intracranial pressure. In cerebral venous thrombosis, rICP may develop due to the increase in venous blood volume, and in some patients, this clinical outcome may be the only or the main finding of cerebral venous thrombosis.

Brain edema can be defined as an abnormal fluid increase in the cerebral parenchyma and the resulting increase in brain volume (3). Cerebral edema due to stroke is classically divided into three groups as cytotoxic, ionic, and vasogenic (Table 1). However, apart from these, interstitial and osmotic edema may also develop in stroke patients, often due to complications that occur during follow-up.

Cerebral edema due to cerebral infarction is seen in both white and gray matter, starts in the first 24 hours, reaches its highest level in 3-5 days, and gradually decreases over the next 2 weeks. Brain edema may develop in all types of ischemic stroke due to etiologies other than small artery occlusion. Malignant brain edema may develop in 

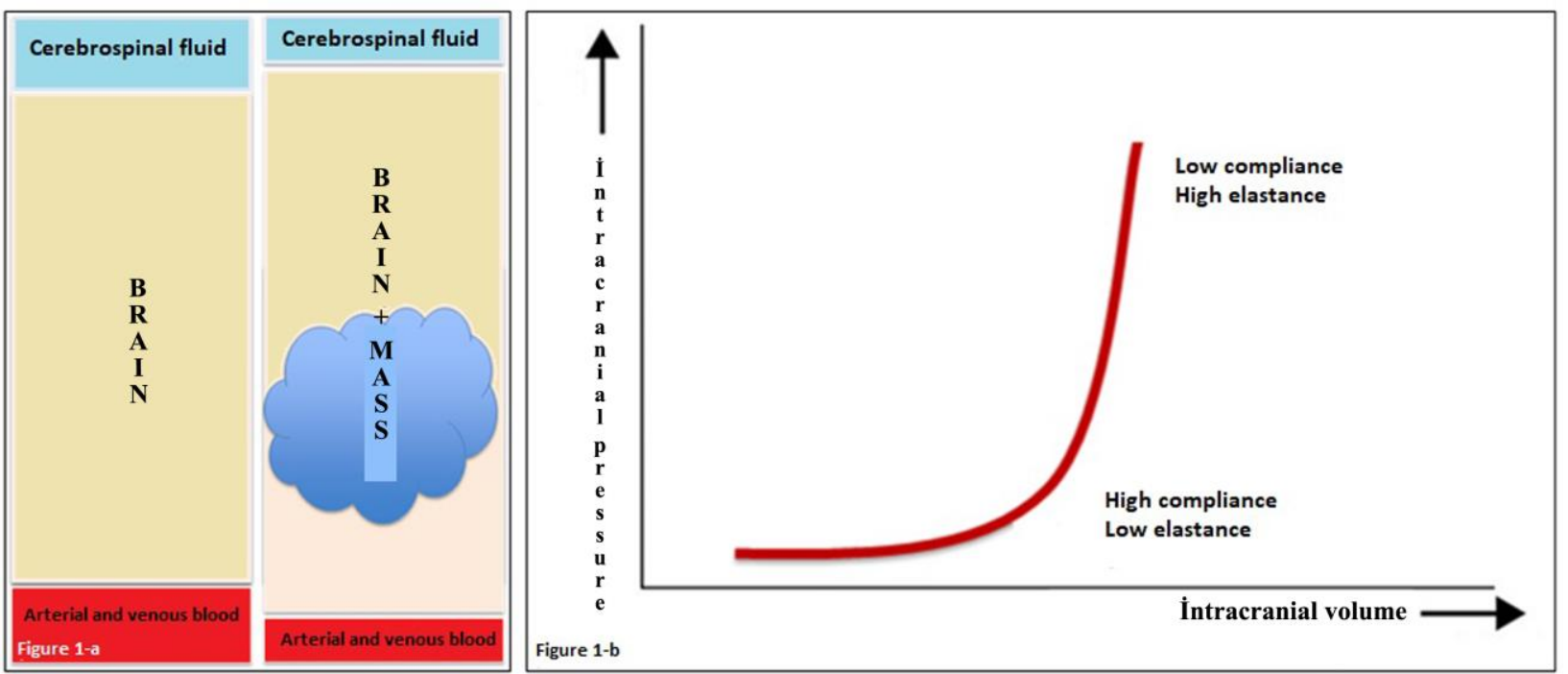

Figure 1: Monro Kellie principle (a) and intracranial pressure-volume relationship (b).

Table 1. Common types of cerebral edema; mechanisms, localizations, and associated conditions.

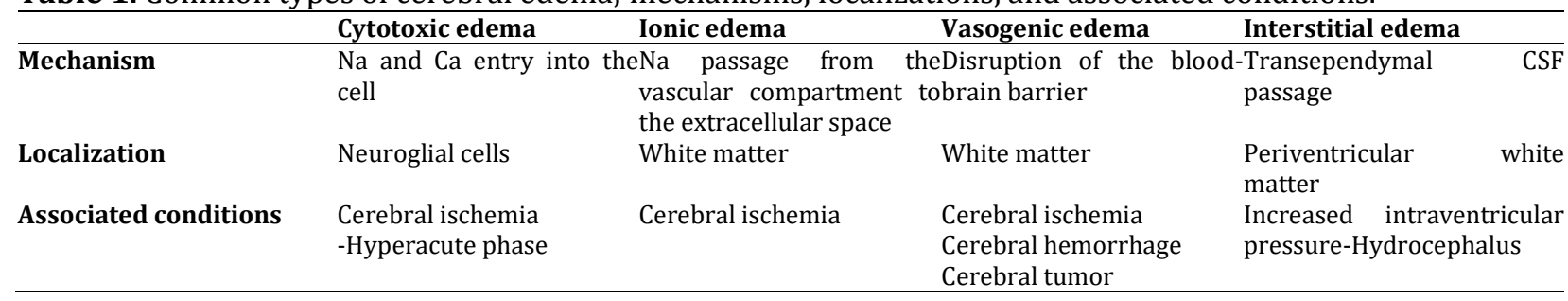

$5-10 \%$ of ischemic strokes. This rate doubles to $30 \%$ in middle cerebral artery (MCA) infarcts resulting from large vessel occlusion (4). In cases where more than $50 \%$ of the middle cerebral artery irrigation area is affected, the risk of developing cerebral edema, rICP, and transtentorial herniation increases. After total MCA infarcts, transtentorial herniation and mortality rate with only medical treatment has been reported as $78 \%(5)$.

Neurological deterioration due to mass effect can be seen in up to $20 \%$ of cases with cerebellar infarction $(6,7)$. Brainstem compression due to ischemic edema may develop in patients with large cerebellar infarction, and acute obstructive hydrocephalus may occur as a result of the blockage of the fourth ventricle. There is a risk of tonsillar herniation and sudden death in cerebellar infarcts and hematomas with mass effect.

In patients with hemorrhagic stroke, rICP may develop due to intracranial volume increase caused by hematoma in the acute period. Disruption of the BBB by the toxic effects of erythrocyte degradation products and hemoglobin is responsible for brain edema and delayed rICP formation $(8,9)$.

\subsection{Effect of Brain Edema on Prognosis in Acute Ischemic Stroke}

Cerebral edema emerges as a life-threatening complication of stroke. It is a major cause of death due to ischemic stroke in the first week. Infarct volume is the most important factor in the formation of edema and herniation. Most stroke patients have some degree of edema. Nausea, vomiting, progressive neurological deficit (hemiparesis, dysarthria, aphasia), and worsening of consciousness in stroke patients may be signs of increasing edema. Edema due to ischemic stroke begins to develop within a short period, and its effect can last from days to weeks. The mass effect is most common in 3rd-5th days, and secondary brain damage and death are most common in these days. After one week, new worsening due to edema is not expected much (10).

Patients with ischemic stroke who develop 
cerebral edema have occlusion of the internal carotid artery or middle cerebral artery. If anterior cerebral artery or posterior cerebral artery occlusion is accompanied, the potential for severe edema is high. No clinically significant edema effect is expected in branch occlusions of the middle cerebral artery alone. The first neuroimaging predicts which patients will develop edema, and these patients often have hemiplegia, head and eye deviation toward the lesion side, global aphasia, neglect, severe dysarthria, visual field defect, and confusion. The most specific sign of cerebral edema exerting pressure on the thalamus and brain stem is impaired consciousness. Pupillary changes typically develop in the 3-5th days and are a sign of severe edema and herniation. Young age, history of hypertension, systolic blood pressure above 180 mmHg, high NIHSS (National Institute of Health Stroke Scale) score at admission, involvement of more than $50 \%$ of the MCA irrigation area on CT, and measurement of large infarct volume in MRI are risk factors for the development of edema (1113).

Neurological deterioration occurs within 72 96 hours in most patients. With the transformation of penumbra tissue at risk into infarct, worsening may take up to 4-10 days in some patients. The incidence of severe edema with middle cerebral artery infarction is 2-8\% among all stroke patients and $10-15 \%$ among patients with MCA infarction. In one study, $78 \%$ of patients with total MCA occlusion developed herniation. The risk of worsening and death increases to $40-80 \%$ in patients with brain edema who become clinically symptomatic. Sometimes the treatment may be insufficient, and brain death may develop in patients connected to a mechanical ventilator due to brain edema $(5,11,14)$.

Brain edema may also develop in posterior fossa infarcts and hemorrhages, and when it develops, it can lead to rapid clinical worsening, herniation, and death. It is difficult to diagnose if the symptoms of cerebellar infarct consist only of dizziness, vertigo, and vomiting. In emergency room conditions, truncal ataxia is a frequently missed finding in patients with poor general condition. There are two dangers of edematous cerebellar infarction or hematomas; compression of the fourth ventricle causing obstructive hydrocephalus and direct brainstem compression. Similar to hemispheric infarcts, the best clinical manifestation of cerebral edema is depression of consciousness and impaired alertness $(11,15)$. If the increase in cerebellar edema cannot be prevented, it may result in an upward transtentorial herniation of the superior vermis through the tentorial opening or a downward herniation of the cerebellar tonsils from the foramen magnum, which is the main cause of mortality in the acute phase (15).

Brainstem compression and obstructive hydrocephalus develop in $10-20 \%$ of patients with cerebellar infarction (16). The mortality rate has been reported as $45 \%$ in patients with cerebellar edema who show a compression effect only with medical treatment, rising up to $85 \%$ in patients presenting with coma (15). Therefore, patients with cerebellar infarction should be followed very closely in stroke units and neurointensive care units. The general treatment of cerebellar infarct is not different from other field infarcts. In contrast to supratentorial infarcts, patients with cerebellar lesions may benefit from posterior decompression surgery, even if their initial state of consciousness is poor (15) (Image 1). In anterior circulation infarcts, it is possible to predict the course of patients with neuroimaging and clinical follow-up. In cerebellar infarcts, weak clinical evidence in some patients with large infarct volume, relatively weak CT in posterior fossa imaging, and rapid worsening of some patients necessitate closer follow up of cerebellar infarcts, and in borderline cases, the patients should be directed to surgical treatment instead of waiting.

\subsection{The Effect of Brain Edema on Prognosis in Intracerebral Hemorrhages}

Intracerebral hemorrhage (ICH) is a subtype of stroke that causes high mortality and disability. While the 30-day mortality ranges from $32-50 \%$, the 1-year survival was reported as $46 \%$ (17). Only $28-35 \%$ of the surviving patients can continue their lives independently in 3 months of termination. The prognosis is associated with the clinical severity at the onset. Rapid diagnosis and early treatment affects prognosis. For these reasons, patients with ICH-associated high mortality and morbidity risk should be well determined and evaluated according to their clinical characteristics.

Perihematomal edema (PHE) grows the fastest in the first 24-72 hours around ICH, comes to the maximum level at 7-10th days and can 

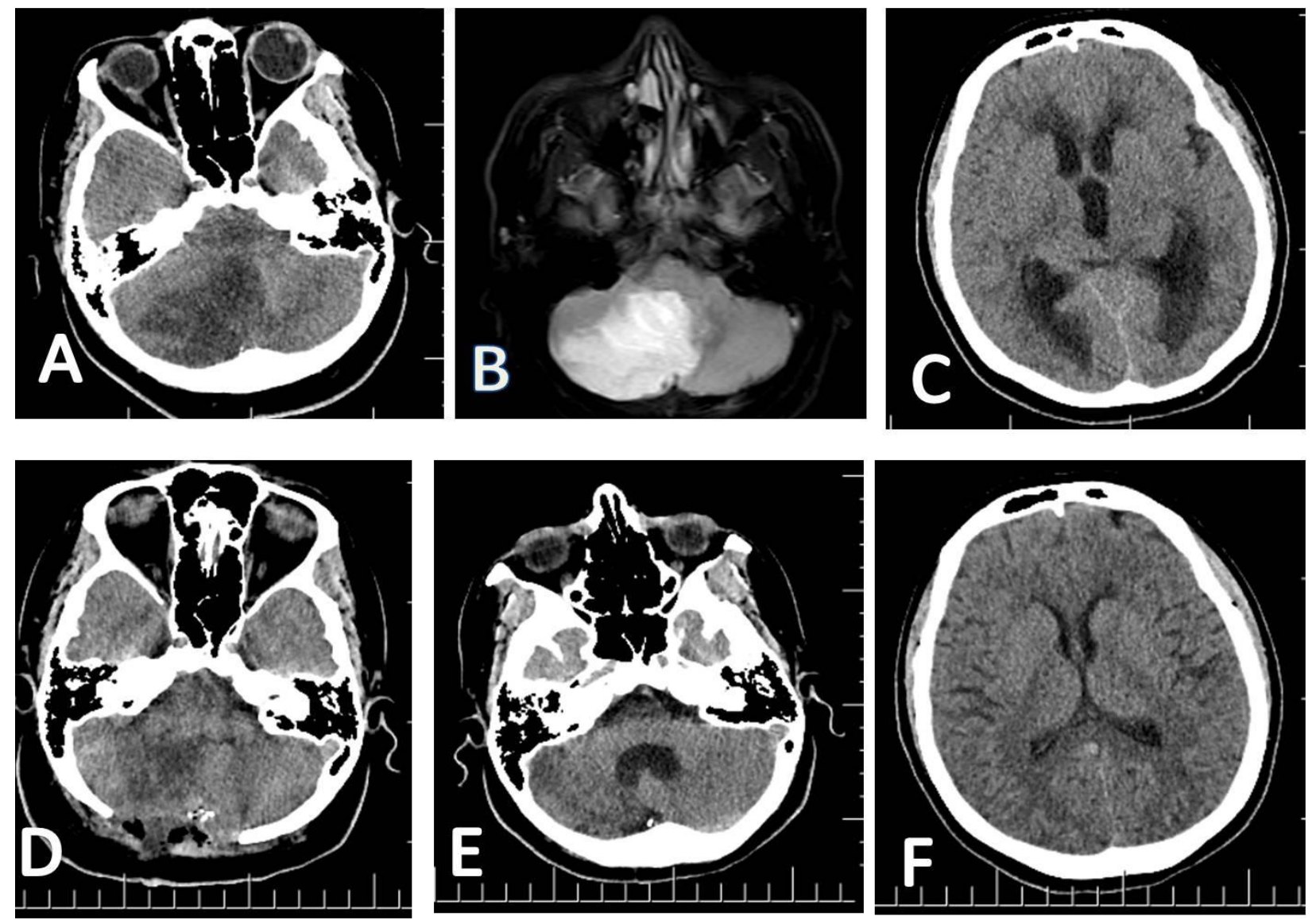

Image 1. CT and MRI (A,B,C) of a 39-year-old female patient showing acute infarction and edema in the cerebellum, blockage in the fourth ventricle, and hydrocephalus. Post-decompression bone defect (D) and control CT 2 months later show that the fourth ventricle is open and hydrocephalus has improved (E, F).

continue to about 14 days (18). In the literature, there are different data on the relationship of PHE with prognosis (19-24). One of the main reasons of this situation is the use of different methods in the measurement and evaluation of edema, such as PHE volume, relative PHE, PHE absolute volume increase, PHE growth rate, PHE absolute area measurement (25). Another reason for heterogeneity is that measurements were made at 24-hour intervals in some studies and 72-hour intervals in others. Besides, severe ICH patients likely to be clinically and radiologically mortal were not included in Intensive Blood Pressure Reduction in Acute Cerebral Hemorrhage Trials 1 and 2 (Interact 1 and 2), the largest series in this subject (24). Therefore, the relationship between PHE and clinical termination might have been reported less than in reality.

In the INTERACT 1 study conducted on 270 patients, even though the baseline volume or 24- hour growth volume of PHE was not associated with the clinical endpoint on the 90th day, this result was attributed to the limited number of patients. Therefore, INTERACT 1 and 2 study data were evaluated and re-analyzed in 1138 patients with a second 24-hour CT scan, revealing that each $5 \mathrm{~mL}$ increase in absolute PHE volume was significantly associated with death or dependency at 90 days. This association was also significant in the multivariate analysis in which various prognostic factors such as demographic features, comorbid cardiovascular risk factors, time to diagnosis, clinical severity, hematoma growth, and randomized treatment. In addition, the significant association of the baseline hematoma volume and 24-hour hematoma growth with the PHE volume expansion was revealed. The NIHSS score, time to baseline CT, location of hematoma, and the use of mannitol were also indicated as independent predictors of the PHE growth. The same study 
determined that the growth of PHE was less in the group receiving intensive blood-pressure (<140/90 mm Hg) treatment (24).

A retrospective analysis of 596 patients has shown the explicit association of PHE volume increase from admission to 72 hours with a poor outcome (26). The midline shift was higher in patients who developed rapid PHE over the 72hour period (27). Another prospective study conducted with 133 patients found that PHE was associated with a poor prognosis in hematomas of $30 \mathrm{ml}$ or less (20).

Intracerebral hemorrhage volume is the main factor determining prognosis. In addition, the increase in intracranial pressure with the development of PHE adversely affects the prognosis as well and should be considered when determining treatment preferences. The increase in PHE is an independent prognostic factor in patients with acute ICH (28).

\subsection{Overview and Pathophysiology of Raised Intracranial Pressure Syndrome}

The Monro-Kellie doctrine states that the total volumes of the three components within the skull (brain, blood, CSF) remain constant, and an increase in one is compensated by a decrease in the other. Exceeding the limits of this compensation system results in rICP. Cerebral edema and rICP that develop during a stroke are seen during both ischemic stroke and intracerebral hemorrhage and cause an increase in poor outcome and death rates in cases of severe pressure elevation $(29,30)$.

How we would help people with increased intracranial pressure was explained 186 years ago by Dr. Alexander Monro, a professor in the Department of Anatomy at Edinburgh University Medical School, like his father and son, with the observations made by Scottish Surgeon Prof George Kellie 11 years earlier. The Monro-Kellie doctrine compares the brain to a 'closed box' and states that in case of an increase in one of the different volumes within a closed box that cannot expand, the volume of the others will decrease (31, 32 ). The skull is a rigid structure that cannot expand in adults. The intracranial volume is approximately $1400-1500 \mathrm{ml}$ in an adult. The volume of the brain is $1150-1250 \mathrm{ml}$. The volume of the intracranial space consists of $80 \%$ brain parenchyma, $10 \% \mathrm{CSF}$, and $10 \%$ blood. The amount of cerebrospinal fluid circulating in the ventricles and the subarachnoid space is $125-150$ $\mathrm{ml}$. The instantaneous blood volume in the brain is $100-150 \mathrm{ml}$, of which about $15 \%$ is in the arteries, $40 \%$ in the veins, and $45 \%$ in the microcirculation $(33,34)$.

Intracranial pressure is measured 5-20 $\mathrm{cmH}_{2} \mathrm{O}$ (3-15 $\mathrm{mmHg}$ ) in a person at rest in the supine position. When the intracranial pressure rises above $20-25 \mathrm{cmH}_{2} \mathrm{O}$ (20 $\mathrm{mmHg}$ ), clinical signs and symptoms and radiological findings appear, and an increase in intracranial pressure is specified. When brain edema develops for any reason and intracranial pressure rises, the primary compensation mechanism is the compression of the ventricles and venous sinuses, pushing the CSF within the cranium out into the subarachnoid space around the spinal cord, into the lumbar cisterns. This primary response attempts to prevent a further increase in intracranial pressure. If the brain continues to swell, the second mechanism to prevent intracranial pressure from rising is to reduce cerebral blood flow $(1,35)$. Arterial pressure in the main arteries of the brain forming the Willis polygon is $50-60 \mathrm{mmHg}$. When the intracranial pressure reaches these values, the cerebral blood flow has come to a standstill. Cerebral blood flow may be impaired at lower intracranial pressure values in the distal arterial bed. Therefore, lowering the intracranial pressure in acute stroke will not only prevent herniation but also avoid any further reduction of intracranial blood flow (36). Since the volume of brain tissue is larger in young patients, fatal brain edema is more common in malignant middle cerebral artery occlusions, and cerebral atrophy observed in elderly patients is considered protective from fatal increased intracranial pressure.

\subsection{Brain Edema Types and Physiopathology in Acute Ischemic Stroke}

Brain edema may develop as a lifethreatening complication in $10-20 \%$ of ischemic strokes. The cause of brain edema is the ionic imbalance that develops due to energy deficiency (5). Brain edema developing after ischemic stroke is mainly divided into two as cytotoxic and vasogenic edema. Cytotoxic edema is caused by the accumulation of fluid in the cell due to damage, while vasogenic edema occurs when the fluid coming out of the blood vessels accumulates around the cells. Cytotoxic edema occurs due to the malfunction of ATP - dependent sodium and 
calcium channels, neurons, glia, and endothelial cells swell due to fluid accumulation in them. During this period, the blood-brain barrier is intact. Vasogenic edema occurs due to the passage of proteins and other macromolecules into the extracellular space as a consequence of the increased permeability after the breakdown of the blood-brain barrier (37).

In the first minutes of ischemia-related damage, cytotoxic edema is observed and develops in neuroglial cells, mainly in astrocytes. When the cerebral blood flow falls below $12 \mathrm{ml}^{1} 100 \mathrm{gr}^{-1}$. $\mathrm{min}^{-}$ 1 , the function of the Na-K ATPase enzyme is impaired; the Na accumulated in the cell cannot be expelled from the cell and accumulates in the cell (3). Fluid enters the cell from the interstitial space, but brain swelling is not expected at this stage, as there is no additional fluid entry into the brain tissue. Potassium accumulates in the interstitial space, then more sodium and calcium flow into the cell under the influence of excitatory neurotransmitters. Due to the disturbed ionic balance, and in order to maintain the disturbed osmotic balance, water enters the cell and the cells swell even more. The receptors and exchangers involved in this process: Sur1-Trpm4 (sulfonylurea receptor 1 -immediate receptor potential melatonin 4), NKCC1 $\left(\mathrm{Na}^{+}-\mathrm{K}^{+}-2 \mathrm{Cl}^{-}\right.$ cotransporter protein), AQP4 (aquaporin-4), $\mathrm{Na}^{+}-$ $\mathrm{H}^{+}$exchanger and $\mathrm{Na}^{+}-\mathrm{Ca}^{2+}$ exchanger (30). Calcium activates inactive phospholipases in the cell, leading to the formation of free radicals and lipid peroxides. The release of arachidonic acid triggers the destruction of the membrane and results in irreversible cell damage. These events occur within minutes in the presence of severe ischemia (brain regions with more than 80\% impaired perfusion compared to pre-occlusion) (38). Cytotoxic edema begins as soon as the cerebral blood flow drops below $30 \mathrm{ml}^{100 \mathrm{gr}^{-}}$ ${ }^{1} \cdot \mathrm{min}^{-1}$ and causes a signal change in $\mathrm{MR}$ as a diffusion restriction (39).

Ionic edema developing in the acute phase of ischemia is seen together with cytotoxic edema (3). When sodium enters the cell, sodium concentration in the interstitial area decreases, leading to sodium transfer from the vascular area to the interstitial space. Ionic edema develops in the interstitial area due to the accompanying influx of fluid with sodium. Unlike cytotoxic edema, edema at this stage is of extracellular type and does not contain albumin. The process is maintained through various ion channels and transporters such as Sur1-Trpm4 channel, NKCC1, $\mathrm{Na}^{+}-\mathrm{K}^{+}$-cotransporter (KCC), $\mathrm{Na}^{+}-\mathrm{H}^{+}$exchanger, and AQP4 (30). During the ionic edema period, the blood-brain barrier is still intact, and the passage of proteins is not allowed (40). At this stage, the blood-brain barrier (BBB) is still intact. İonic edema is most commonly seen after ischemic stroke, causing an increase in brain parenchymal volume and intracranial pressure.

There are tight junctions between capillary endothelial cells in the brain, and typical capillary fenestrations in the basal lamina are not found in brain capillaries (41). Pericytes and astrocytic endfeet support tight junctions between endothelial cells. Vasogenic edema develops as a result of disruption of the blood-brain barrier after cerebral ischemia or hemorrhage (42). Vasogenic edema develops following cytotoxic edema in the acute phase of cerebral ischemia, about 4 to 6 hours after the onset of ischemia. In the late stage (after a few days), there is a separate period of vasogenic edema. The second period is more decisive for the development of rICP. Processes that increase intravascular pressure or cause vasodilation may increase vasogenic edema. White matter is affected more by vasogenic edema due to its loose structure, and edema progresses between axons. In the cerebral cortex, which is formed in the neuronal bodies, the cell density and intercellular connections are higher, and therefore the edema cannot find a way to spread. Tissue water content increases by $15 \%$ in cerebral cortex and $100 \%$ in white matter due to vasogenic edema (38).

The mechanism by which ischemia disrupts the blood-brain barrier is not clearly known. In the case of ischemia, active pinocytosis (intake of fluid by vesicles into the cell) is first observed in endothelial cells, and the deterioration of tight junctions occurs at a later stage.

Additionally, ischemia-induced matrix metalloproteinases, nitric oxide synthetase, vascular endothelial growth factors, and thrombin may also be associated with blood-brain barrier disruption $(40,43)$. On the other hand, at this stage, AQP4 plays a role in the clearance of vasogenic edema $(30,44)$. The excessive increase of tissue fluid during vasogenic edema can induce secondary ischemic damage, in addition to rICP, herniation, and capillary perfusion pressure (39) (Figure 2). 


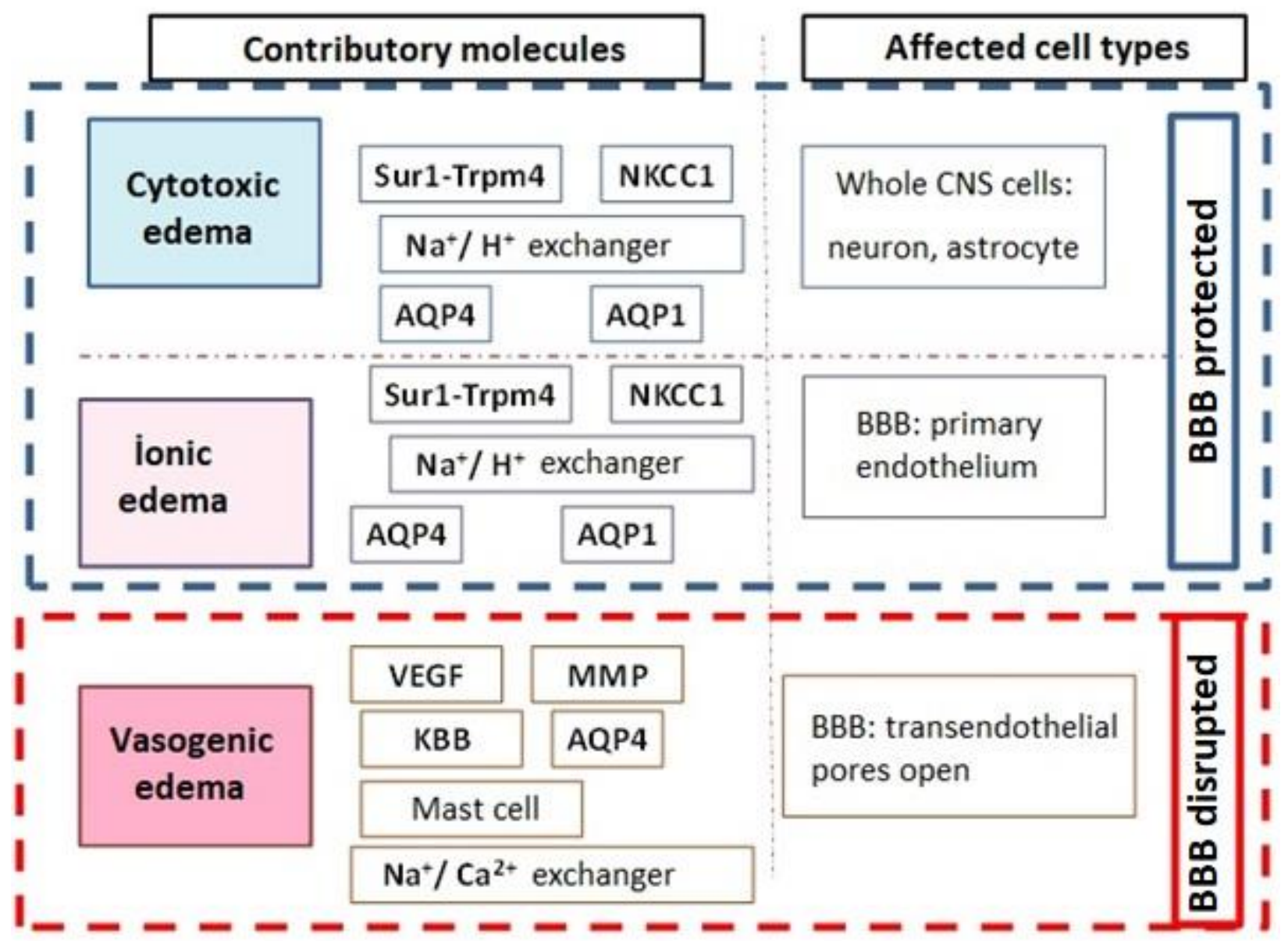

Figure 2. Overview and physiopathology of brain edema types developing after ischemic stroke.

Interstitial (transependymal) edema is another type of brain edema seen in hydrocephalus rather than cerebral ischemia. It occurs with increased intraventricular pressure and is due to transependymal CSF flow. CSF infiltrates the extracellular space in the white matter adjacent to the ventricles. It is most commonly seen in acute obstructive hydrocephalus. On the other hand, hypoosmotic or osmotic edema develops due to low plasma osmolality. Hypervolemia may develop as dilutional hypervolemic hyponatremia due to inappropriate antidiuretic hormone syndrome, as hypovolemic hyponatremia due to cerebral salt loss, or as iatrogenic (infusion of hypotonic fluids, hemodialysis, etc.). In osmotic edema, serum osmolality decreases, and fluid flows into the extracellular space. Extracellular $\mathrm{Na}$ decreases, and intracellular $\mathrm{Na}$ increases relatively. In osmotic edema, cytotoxic edema may also develop, but BBB disruption has not been demonstrated (45).

\subsubsection{The Blood-Brain Barrier And The Neurovascular Unit}

Although the brain makes up only a small part of our body, it uses $20 \%$ of all our energy at rest, making it our biggest source of energy consumption (46). Normal brain function requires the replenishment of ATP stores after neuronal activation. The main energy sources required for ATP production are oxygen and glucose, which must be continuously provided by the bloodstream and transported to the brain. This event takes place in the microcirculation. Capillaries make up $85 \%$ of the vessel length in the human brain, providing a total of $12-18 \mathrm{~m}^{2}$ surface area. Therefore, this large surface area formed by 
the capillaries constitutes the main interface for the exchange of substances between the blood and the brain $(47,48)$. The most critical structure in this interface is a unique region called the BloodBrain Barrier (BBB). Anatomically, the BBB can be thought of as a highly selective semipermeable membrane at the capillary level. Apart from this structural function, it also has other important functions such as controlling the chemical composition of the neuroglial environment, regulating the transport of molecules from the blood to the central nervous system (CNS) and from the CNS to the blood, and preventing the entry of toxic plasma components, blood cells or pathogens into the brain (49).

Considering the regional differences in brain activity and dynamic energy requirements, it is important that the blood flow reaches the right place and in the right amount at the right time. Cerebral arteries, arterioles, and capillaries provide blood flow to the CNS circuits through a mechanism called neurovascular coupling. This mechanism involves a delicate flow regulation that directs blood flow and oxygen to activated neuronal groups while reducing blood flow to nonactivated areas $(50,51)$. The dynamic anatomical structure that provides this mechanism is called the Neurovascular Unit (NVU). The NVU represents both the functional and structural multicellular relationship between the brain and blood vessels in microcirculation. NVU, a concept formalized in 2001 in neuroscience, contributes to the regulation of $\mathrm{BBB}$ permeability, cell-matrix interactions, neurotransmitter cycling, angiogenesis, and neurogenesis, apart from the neurovascular coupling $(48,52,53)$. The neurovascular unit at the capillary level is comprised of endothelial cells, mural cells (pericytes and vascular smooth muscle cells), glial cells (astrocytes ,microglia) and neurons (Figure 3).

The blood-brain barrier is at the center of the NVU $(48,49)$. The shielding properties of the blood-brain barrier make brain capillaries different from those in other tissues of the body, and the structure is shaped by a network of protein complexes, cellular properties, and interactions between cells. Brain endothelial cells

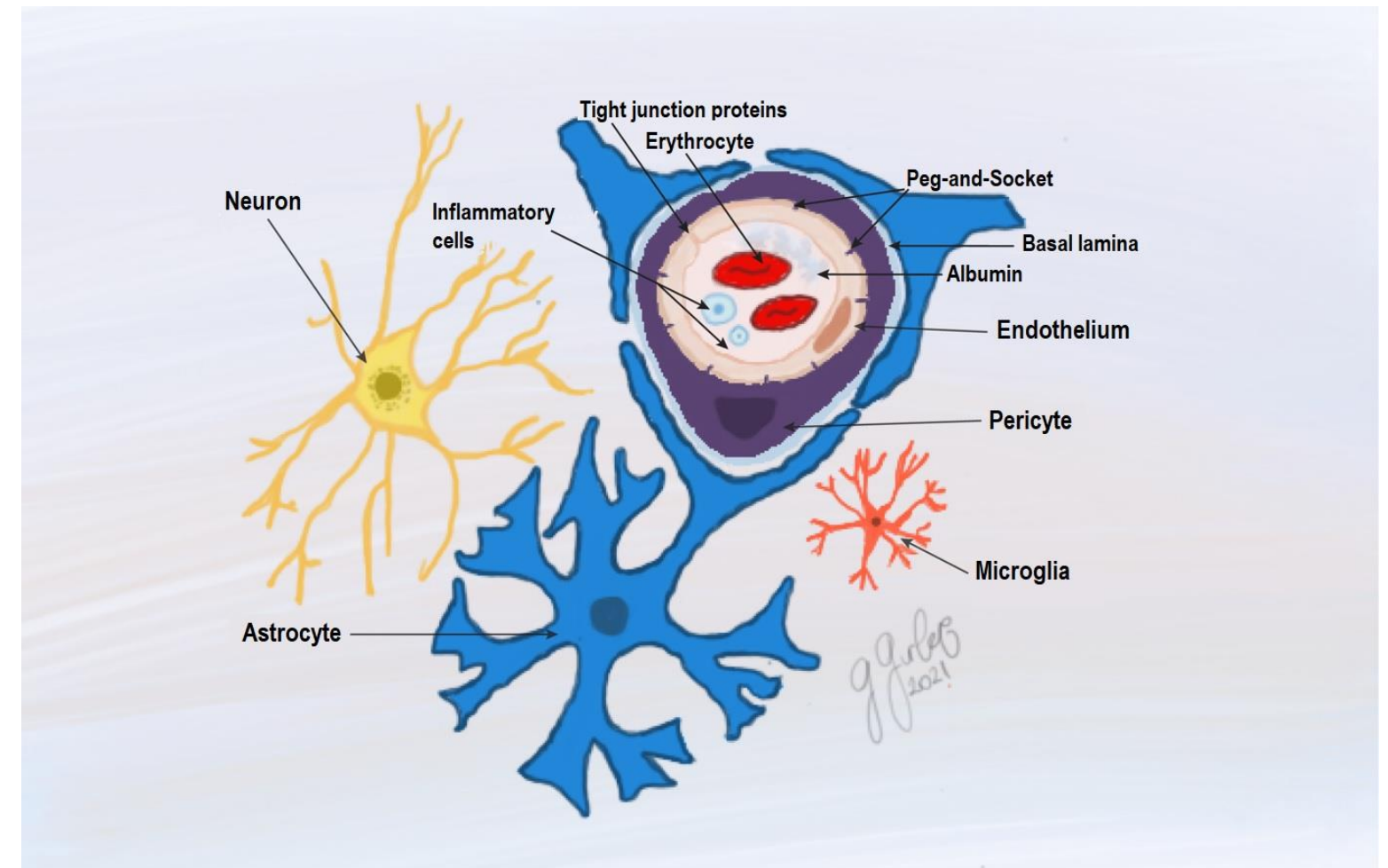

Figure 3. Neurovascular Unit. The structures that make up the NVU are shown schematically in the capillary section. Endothelial cells sealed with tight junction proteins, pericytes in the basal lamina, astrocytes surrounding the microvessel with their endfeet, microglia, and neuron are shown. There are peg-and-socket connections between the endothelium and the pericyte. 
differ from those in the peripheral vasculature: they contain abundant mitochondria, no fenestrations, and fewer caveolae (specialized lipid rafts) that restrict pinocytosis. In addition, they form a capillary wall structure in the form of a continuous endothelial cell membrane, virtually sealed with each other, thanks to special tight junction proteins (claudins, occludins, and adherins) that restrict paracellular (transfer of substances between cells) passage $(47,54,55)$. Pericytes, which surround the capillaries with their extensions, contribute to the development and maintenance of the $\operatorname{BBB}(48,54,55)$. In addition, they have contractile properties with their unique cellular skeletal structures consisting of actin filaments and can change vessel diameters and thereby play an important role in neurovascular coupling $(50,56)$.

In cases where blood flow is not sufficient, such as ischemic stroke, not only neurons but also vessels are affected, and BBB functions are impaired. This disruption exacerbates postischemic edema by causing extravasation of bloodderived proteins and water (57). In the formation of the tight junction protein complex between BBB function and endothelium, cellular interactions with not only vascular endothelial cells but also pericytes and astrocytes are critical, and their functions are all impaired by ischemia (58-60). Both clinical and experimental studies have shown that BBB disruption causes a poor prognosis.

\subsubsection{Inter-Endothelial Tight Junctions}

Endothelial cells are the cells lining the inner wall, facing the inferior surface of the vessel in all vascular structures in our body. However, there are some specialized structures in the nervous system that distinguish these cells from other cells. One of them is the tight junction structures that anchor two endothelial cells to each other. While the transition from blood to the organ and from organ to blood is quite loose in other organs due to the small number of tight junctions between the endothelium, this transition is tightly controlled in the nervous system. Tight junction proteins are the most important structures in this controlled passage or gate function and play a pivotal role in the blood-brain barrier formation. This structure was first described in the 1880s by Paul Ehrlich. Ehrlich proposed that such a structure should exist by showing that the dyes injected into the blood in his experiments did not pass into the central nervous system (61). Today, thanks to the developing microscopy and examination techniques, we know the tight junctions in detail.

In the central nervous system, some specific properties allow endothelial cells in the vessels to act as a barrier. These are;

1. Tight junctions and adherens junctions

2. Containing few pinocytic vesicles and many mitochondria.

If we take a closer look at the tight junction structures, it is seen that endothelial cells are connected to each other and are located on the surface facing the blood $(62,63)$ (Figure 4).

Tight junction structures consist of occludins, claudins (especially $-5,-3$, and -12 ), and zonula occludens proteins attaching them to endothelial cytoplasmic structural proteins, as well as adherens junction proteins. These structures form a barrier for small molecules that dissolve in water and can pass easily between cells (paracellular) through the nervous system (64-66).

In addition to tight junction proteins, there are also junctional adhesion molecules (JAM) located in the basal region between the two endothelium. Junctional adhesion molecules mainly provide structural support and are formed by the adhesion protein JAM. The cadherin and catenin proteins, to which it binds in the cytoplasm, are other adhesion molecules, and together with JAM, help to form this adhesive structure. While tight junctions have a more dynamic feature due to phosphorylation and mechanical effects, adhesion molecules are more stable.

Tight junctions have a significant role in the blood-brain barrier formation, and its damage and/or loss of function causes fluid transfer to the central nervous system, in other words, vasogenic brain edema. Indeed, the knockout of claudin proteins by gene deletion technology disrupts the blood-brain barrier, making it leaky, and causes edema (67). It is known that following the damage of these structures during ischemia-reperfusion in ischemic stroke, the uncontrolled fluid transfer to the brain tissue results in brain edema $(66,68)$.

Tight junction proteins can be affected at various levels in stroke. One of them is phosphorylation. Occludin and claudins are phosphoproteins, and their phosphorylation determines their tight junction activity. It has been shown that inflammation, increased and/or decreased phosphorylation in stroke cause this 
structure to have a hyperpermeable property $(69$, 70). Likewise, zonula occludens proteins located on the cytoplasm side loosen with phosphorylation and cause an increase in permeability (especially in viral infections). Besides, following ischemia, tight junction proteins are internalized by a caveolin-mediated system and transported to the cytoplasm (71). In addition to all these, several studies have demonstrated that there is increased matrix metalloproteinase (MMP) secretion from various cells of the neurovascular unit in ischemia and especially MMP-9 and MMP-2 increase from the early hours of stroke and cause an increase in blood-brain barrier permeability by breaking down tight junctions $(66,68,72)$.
In the early post-stroke period, cytotoxic edema occurs due to the swelling of cells as a result of energy deprivation, followed by vasogenic edema caused by uncontrolled fluid flow from the blood due to the disruption of the bloodbrain barrier elements, mainly tight junction proteins. Various centers are conducting studies on the mechanisms that prevent and/or reverse the changes in tight junctions mentioned above and searching for possible new treatment options to be developed (Rho/Rho-associated protein kinase -ROCK inhibitors (such as fasudil and MMP inhibitors) (73). As our knowledge of the functioning of tight junction structures increases, our effective strategies for reducing edema and damage in stroke will increase in the coming years.

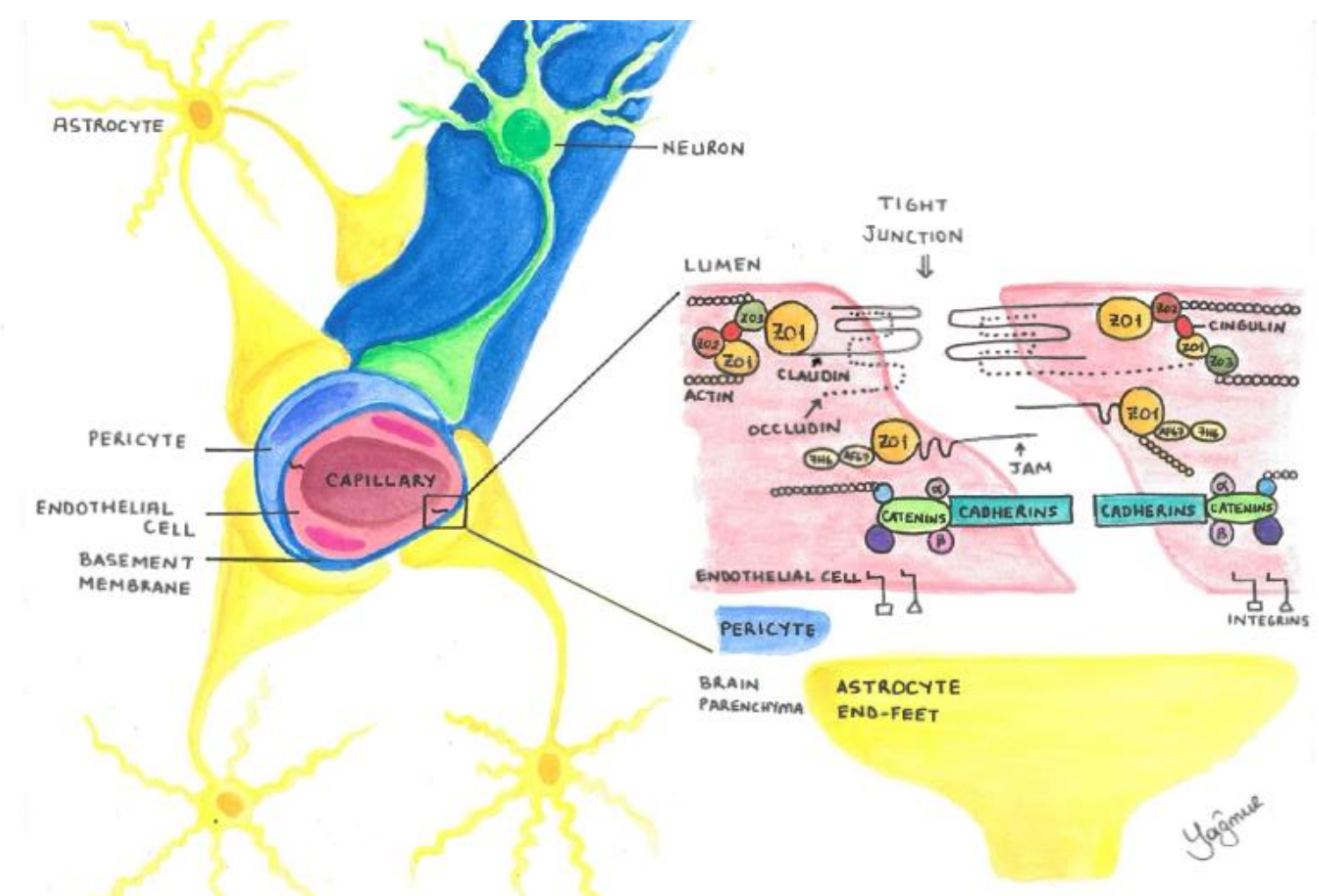

Figure 4. Tight junctions within the neurovascular unit. On the surface interacting with blood, tight junctional elements are apically located.

\subsubsection{NKCC1 and Cerebral Edema}

NKCC (Na-K-Cl Cotransporter) are membrane proteins that transport sodium and potassium ions along with chloride into and out of the cell across the cell plasma membrane $(74,75)$. It has two isoforms, NKCC1 and NKCC2 $(74,76)$. NKCC1 has a broad tissue distribution, while the NKCC2 isoform

Turkish Journal of Cerebrovascular Diseases 2021; 27(2): 65-132 is found only in the kidney of vertebrates (74-77). Immunohistological studies have shown NKCC1 expression in neurons, astrocytes, oligodendrocytes, choroid plexus, and endothelial cells forming the BBB (78-81). NKCC has different functions in tissues, such as secretion and reabsorption of ions and fluids and regulation of cell volume and sodium content $(82,83)$. 
It is well established that neuronal death caused by ischemia-induced oxygen-glucose deprivation in neurons is mediated by excitotoxicity triggered by NMDA receptors (84). High extracellular $\mathrm{K}^{+}$and glutamate levels that occur in cerebral ischemia stimulate NKCC1 expression in neurons and astrocytes via NMDA and AMPA receptors $(76,85-87)$. Increased NKCC1 expression leads to excessive $\mathrm{Na}^{+}$influx along with $\mathrm{Cl}^{-}$into the cell (83). High extracellular $\mathrm{K}^{+}$level triggers NKCC1 activity, causing swelling of astrocytes and contributes to brain edema (85, 86). Excessive $\mathrm{Na}^{+}$entry into neurons and astrocytes increases the intracellular osmotic pressure and causes the water in the intercellular space to enter the cell, and cytotoxic edema develops.

Excess $\mathrm{Na}^{+}$accumulation in the cell allows calcium to enter the cell, this time with the reverse operation of the $\mathrm{Na}-\mathrm{Ca}$ exchanger (89). The $\mathrm{Na}-\mathrm{Ca}$ exchanger normally extrudes $1 \mathrm{Ca}^{+}$versus the flow of $3 \mathrm{Na}^{+}$, thus transferring 1 positive charge to the cell. Besides the $\mathrm{Na}^{+}$increase, depolarization of the membrane during ischemia may also cause the $\mathrm{Na}$ $\mathrm{Ca}$ exchanger to operate in the reverse direction due to its electrogenicity (88). On the other hand, it has been shown that $\mathrm{Na}$-Ca exchanger blockers (bepridil, benzamil, dichlorobenzamil) protect the optic nerve from anoxic injury (89).

Bumetanide, a loop diuretic from the sulfamoylbenzoic acid group, inhibits NKCC1 function by competing with $\mathrm{Cl}^{-}$for the second binding site. Bumetanide is the most commonly used drug for NKCC1 inhibition, but furosemide, another loop diuretic from the same group, also inhibits NKCC1 (84). Blocking NKCC1 activity with bumetanide abolishes $\mathrm{Cl}^{-}$accumulation and reduces $\mathrm{Na}^{+}$increase by $50 \%$. Bumetanide also inhibits both glutamate and NMDA-mediated cell death (83). It is anticipated that blocking NKCC1 activity may reduce cell death by preventing neuronal swelling during ischemia. The role of NKCC1 in ENT endothelial cells is crucial. Intravenous administration of bumetanide significantly reduced edema formation in rat stroke models $(82,90)$.

In the study by Huang et al., STS66 (3(Butylamino) - 2 - phenoxy - 5 - [(2,2,2trifluoroethylamino)methyl] benzenesulfonamide), a new NKCC1 inhibitor in mice, was found superior to bumetanide in reducing ischemic infarct area, cellular swelling, and neurological deficit in transient ischemic stroke with large artery atherosclerosis and ischemic stroke accompanied by hypertension (91).

In conclusion, NKCC1 plays a role in cerebral edema formation. Pharmacological inhibition of NKCC1 reduces ischemia-induced cellular swelling, BBB destruction, cerebral edema, and neurotoxicity (83, 85-87, 90, 92-94). Pharmacological strategies aimed at inhibiting NKCC1 may be beneficial for the treatment of ischemic cerebral edema.

\subsubsection{Sur1-Trpm4 Channels and Cerebral} Edema

The sulfonylurea receptor 1 (Sur1) is a member of the adenosine triphosphate binding cassette $(A B C)$ transporter superfamily. ABC transporters use the energy obtained from ATP hydrolysis for various cellular functions. Sur1 and Sur2 are unique among $\mathrm{ABC}$ transporters in that they serve as ion channel regulators (95). SUR1 alone does not have a transport function; it interacts with different membrane proteins to modulate the operation of ion channels. Sur1, encoded by the Abcc8 gene, combines with different proteins in different cell types; for example, with the Kir 6.2 protein to form ATPdependent $\mathrm{K}^{+}$channels or with Trpm4 to form Sur1-Trpm4 channels $(96,97)$.

Transient receptor potential (TRP) channels constitute a family of 28 different cation channels found in mammals. Unlike other Trp channels, the Transient receptor potential channel melastatin 4 (Trpm4) is impermeable to calcium and carries monovalent cations such as sodium excessively and non-selectively. Trpm 4 channels are activated by an increase in cytosolic $\mathrm{Ca}^{++}$or a decrease in cytosolic ATP $(98,99)$.

Increased expression of the Sur1-Trpm4 channel was first demonstrated in 2001 in an experimental rat model in reactive astrocytes in the core region of ischemia (100). Sur1 is the regulatory subunit of this ion channel, and Trpm4 is the pore-forming subunit (Figure 5). Under physiological conditions, Sur1 is observed in neurons but not in astrocytes and endothelial cells. However, it has been shown that Sur1 protein and mRNA increase in neurons, astrocytes, and endothelial cell samples in the presence of cerebral ischemia. Increased expression of Sur1 or Trpm4 in different neurological diseases, such as traumatic brain injury models, is associated with 
the risk of cerebral edema $(97,101-108)$. In 2020, Woo SK et al. confirmed with a study using specific antisense oligodeoxynucleotides that edema developing after ischemia is caused by Sur1Trpm4 channel modulation, not by ATP-dependent $\mathrm{K}^{+}$channels (107). The sur1-Trpm4 expression occurs in the ischemic chord at the 3rd hour and penumbra at the 8th hour after middle cerebral artery occlusion (109).

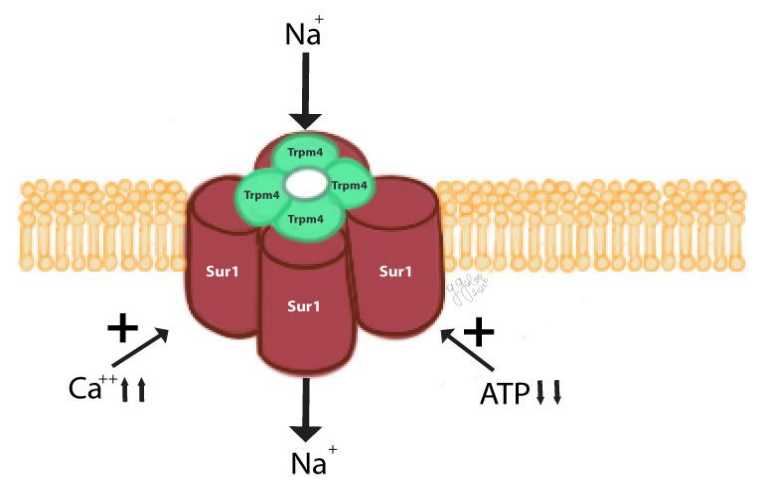

Figure 5. Structure of the Sur1-Trpm 4 channel.

ATP depletion or increase in $\mathrm{Ca}^{++}$ concentration rapidly activates Sur1-Trpm4 (100). Sur1-Trpm4, activated in acute injury, initially slows down $\mathrm{Ca}^{++}$entry to protect the cell from excessive $\mathrm{Ca}^{++}$increase (96). Sustained activation of Sur1-Trpm4 by severe ATP reduction causes $\mathrm{Na}^{+}$influx into the cell and hence cell depolarization. As water also enters the cell, the accumulation of $\mathrm{Na}^{+}$in the cell turns into a maladaptive process that leads to cell swelling (cytotoxic edema) and osmotic cell death (96). In addition to the formation of ionic edema with the increase in $\mathrm{Na}^{+}$in neurons and glial cells, increased $\mathrm{Na}^{+}$in the endothelial cells in the ischemia region causes cytotoxic edema, disruption in the endothelial cytoskeleton, loss of endothelial tight junction integrity, and consequently, the addition of vasogenic edema to the clinical picture $(40$, 110). The extravasation of TNF alpha, immunoglobulin G, and myeloperoxidase (MPO) detected in the microvascular bed with increased expression of Sur1-Trpm4 also proves the development of inflammation and vasogenic edema (99). Nevertheless, there are also studies showing that Sur1-Trpm4 causes astrocytic swelling by forming a heteromultimeric water/ion channel complex with aquaporin 4 (44).

Turkish Journal of Cerebrovascular Diseases 2021; 27(2): 65-132

\subsubsection{The Role of Aquaporin Channels in} Cerebral Edema

Extremely rapid fluid transport is required in many human tissues. This rapid flow of water in tissues is possible in two ways; first, transcellular water flow across the basal and apical membranes in response to osmotic stimuli produced by salt transport, and second, paracellular water passage from intercellular connections to the intercellular space induced by salt or solute gradients. Aquaporins (AQPs) are water channels that regulate transcellular water passage across cell membranes. Aquaporin channels are present in the retina, olfactory epithelium, inner ear, spinal cord, intervertebral disc, osteoclasts, endothelium, heart, salivary glands, gastrointestinal tract, liver, pancreas, lungs, ovaries, sperm cells, adipocytes, and skin. Seven types of aquaporins have been identified in the brain, but the most prominent are aquaporin type 4 in astrocytes and aquaporin type 1 in the choroid plexus $(24,111)$ (Table 2).

Aquaporins consist of six transmembrane proteins in a helical structure attached each other by NPA (asparagine-proline-alanine) sequences. The protein sequence has cytoplasmic free carboxyl and amino terminals. These helical proteins extending from inside the cell membrane to the outside rotate 360 degrees around themselves and come together to form the aquaporin channel with a pore in the middle. Monomer aquaporin channels bind each other to form the tetramer structure (Figure 6). The shape of the aquaporin channel core in the middle acts as a filter and allows only one water molecule to pass (112-115).

Among AQPs, cerebral edema pathophysiology is at most associated with AQP4. AQP4 is the main water transport channel in the central nervous system and is present in high amounts in the astrocyte endfeet that join the blood-brain barrier. It regulates the passage of water across the blood-brain barrier in both directions, that is, from the endovascular space to the intercellular compartment, and from the extracellular space to the endovascular space (115-117). AQP4 expression increases at the 48th hour after ischemia on astrocytes at the border of the infarct and in the astrocyte endfeet at the core and border of the infarct at the end of the first week, that is, in the regions where edema is most prominent (118). This increase is also associated 
Table 2: Experimental studies investigating the effect of AQP4 in different neurological diseases.

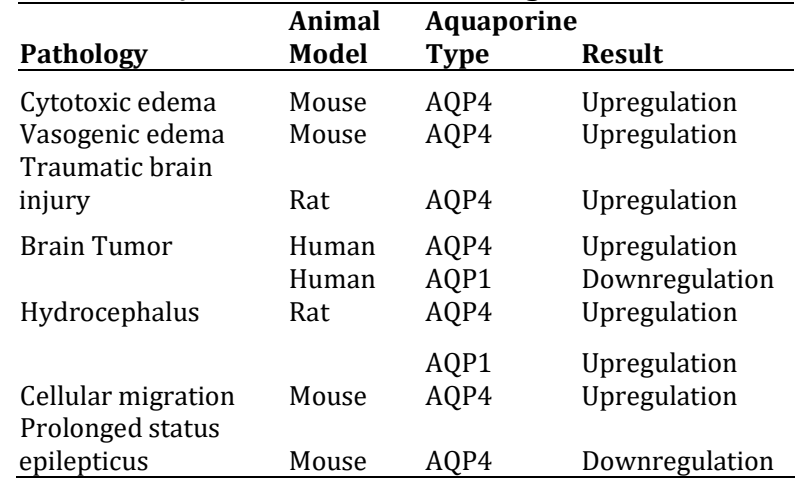

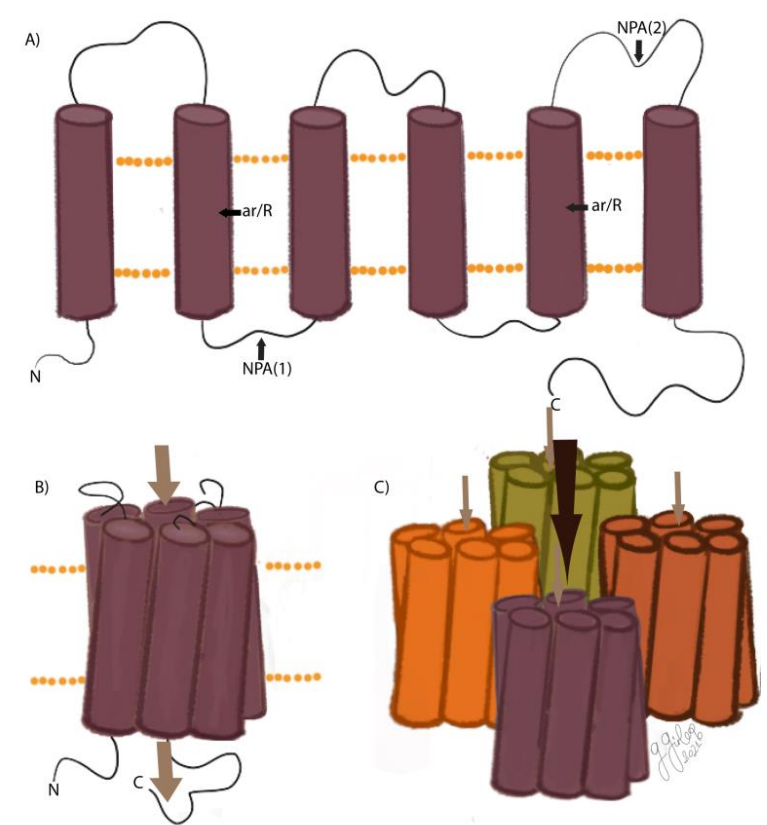

Figure 6. Structure of the aquaporin channel; NPA sequences and six single-helical transmembrane proteins linked together (A) form monomers (B). The four monomers come together to form the tetrameric channel (C).

with local levels of inflammatory cytokines (119122). AQP4 levels return to normal on the 28th day. Studies in AQP4 knockout rodents have shown that in the absence of AQP4, the infarct volume is smaller, blood brain barrier dysfunction and local neuroinflammation are less, neurologic recovery is better, and cytotoxic edema does not develop $(58,115,123,124)$. It has been observed that the AQP4 isoform increases by 2-6 fold with edema in ischemic white matter in humans, but the same increase does not occur in the cerebral cortex, where cellular swelling is very low (125).
In experimental models, mitogen-activated protein kinase (MAPK) pathways are considered effective in the increase in AQP4 synthesis in astrocytes in the ischemia area $(115,126)$. On the other hand, another study reported in a temporary middle cerebral artery occlusion model that the edema detected by MR between 3-7 days was higher in mice lacking the AQP4 gene (127). Aquaporin 4 has two isoforms in the brain, AQP4-M1 and AQP4M23. The AQP4-M1 isoform also appears to reduce brain edema in the experimental model of cerebral ischemia. It is anticipated that AQP4 provides the transport of water in the intercellular space to the endovascular compartment and clearing the edema, especially in the late stages. The effect of AQP4 in the early stages of cerebral edema developing after ischemia is actually dual and complex, depending on the time elapsed since the infarct, the degree of damage, the type of edema that is evident, and the measurement method used. It is considered to be particularly related to the dominant M1 or M23 isoforms (115).

AQP9 increases in ischemic brain tissue at the 24th hour, but it is not associated with cellular swelling. Few studies also provide evidence of an association between cerebral ischemia and AQP3, $\mathrm{AQP5}$, and AQP8 (122).

AQP4 has also been studied in experimental models of intracranial hemorrhage. Although models such as autologous blood injection, bacterial collagen injection, or spontaneous bleeding do not fully mimic human spontaneous intracerebral hemorrhage, it is interesting that AQP4 expression increases at 3 hours after injection, reaches a maximum in 2-5 days, and disappears after 14 days. Moreover, sometime after bleeding, the membrane AQP4 protein is internalized and degraded by lysosomes within astrocytes. Experimental knockout of AQP4 aggravates brain edema that develops after hemorrhage. AQP4 appears to contribute to the clearance of vasogenic edema after intracerebral hemorrhage (115).

Several experimental studies investigating the effect of AQP4 in different neurological diseases are summarized in Table 2.

\section{Edema}

2.2.6 Different Aspects of Reperfusion

In acute ischemic stroke, reperfusion with thrombolytic therapy or mechanical thrombectomy often provides functional 
improvement. However, in some cases, successful recanalization and reperfusion may paradoxically cause more damage in the ischemic tissue than the damage caused by ischemia. This clinical picture is called reperfusion injury, and it is among the important causes of early neurological deterioration due to the development of vasogenic edema and intracerebral hemorrhage (128, 129). In reperfusion injury, mortality is $36-63 \%$, and morbidity is $80 \%$. Although reperfusion-induced edema is clinically associated with recanalized large vessel occlusions, it can also occur after intravenous recombinant tissue plasminogen activator therapy, carotid endarterectomy, intracranial stenting, and even spontaneous recanalization (128).

In the early period after reperfusion, autoregulation is impaired due to excessive vasodilation in the collateral circulation, and luxury perfusion may occur. The relationship of luxury perfusion with either hyperperfusion and reperfusion edema is still unclear (Image 2).

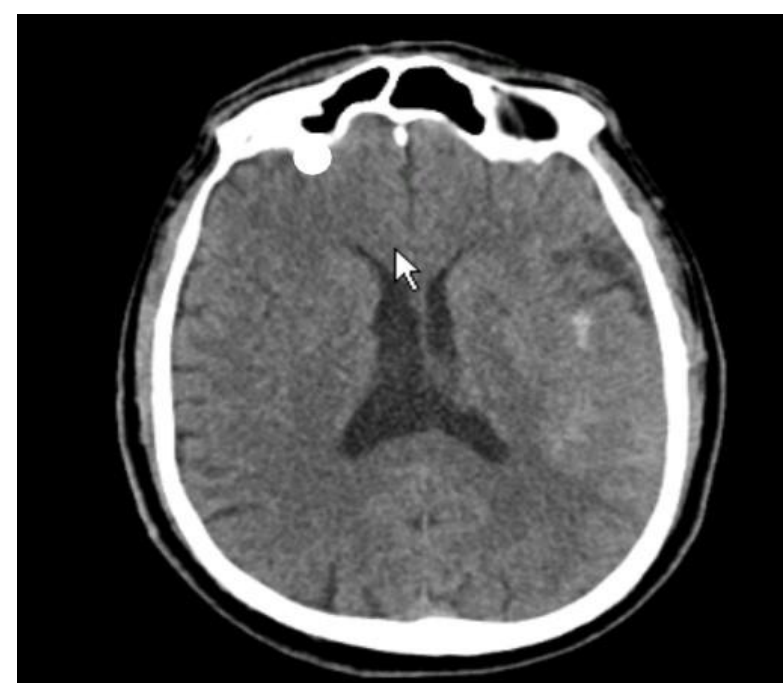

Image 2. Luxury perfusion in the left hemisphere 24 hours after recanalization.

Ischemic tissue damage continues to increase with re-oxygenation of the damaged tissue when reperfusion is achieved. After reperfusion, the brain tissue obtaining an immediate source of molecular oxygen produces reactive oxygen radicals such as hydroxyl radical $(\mathrm{OH})$, superoxide radical $\left(\mathrm{O}_{2}\right)$, and hydrogen peroxide $\left(\mathrm{H}_{2} \mathrm{O}_{2}\right)$. Oxidative stress caused by these free radicals damages endothelial cells. Increasing leukocyte infiltration triggers inflammation. A large number of cytokines, chemokines, inflammatory factors such as NO, TNF-a, IL-1b, IL-6, prostanoids are released from active astrocytes and microglia. The resulting inflammatory response is highly cytotoxic and increases neuronal death in the penumbra. In addition, pericytes contract continuously, narrowing the microvascular lumen and increase microvascular thrombosis with the activation of platelets (130). The integrity of the blood-brain barrier is disrupted, and the infarct volume increases (131). Some inflammatory cytokines are closely associated with the expression of matrix metalloproteinase-9 (MMP9) and aquaporin 4 (AQP4). MMP9 activation disrupts the blood-brain barrier and damages the neurovascular unit. AQP4, on the other hand, increases cerebral edema by facilitating the passage of water to the brain.

Disruption of the blood-brain barrier, composed of endothelial cells, pericytes, astrocytes, and neurons, with increased oxidative stress may increase cerebral vascular permeability and lead to brain edema formation (132, 133). Animal experiments have shown that vasogenic edema develops with increased blood-brain barrier permeability after reperfusion, yet it is a complex process that depends on many factors such as the severity of ischemia, its duration, and reperfusion time (134).

Reperfusion-induced brain edema may present with ipsilateral headache, contralateral neurologic deficit, and epileptic seizures. Symptoms may appear immediately after recanalization or may be delayed for up to 1 month. Patients are usually symptomatic within the first week (Image 3).

Delayed revascularization minimizes benefit and presumably increases the risk of reperfusion injury. The purpose of acute revascularization should not only be to open the vessel, but also to open it quickly (135).

Patient selection based on physiological parameters is critical in reducing edema due to reperfusion. The use of perfusion images to detect salvaged brain tissue and ischemic nuclei, faster recanalization, and the use of new generation retractable stents will increase the success of treatment (136). Especially patients with small ischemic nuclei and large penumbra benefit more from treatment. The extent of vasogenic edema is 


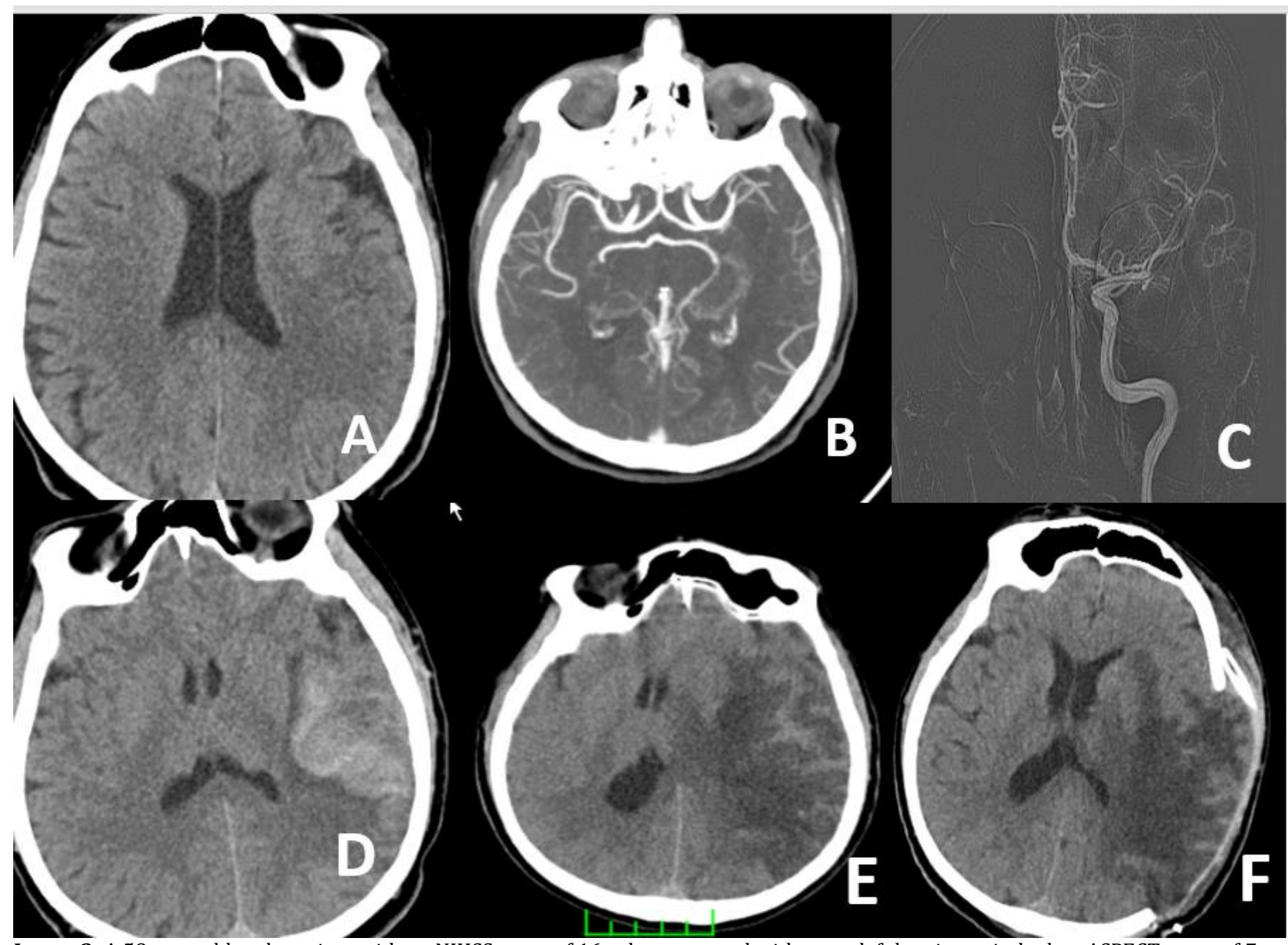

Image 3. A 58-year-old male patient with an NIHSS score of 16, who presented with acute left hemiparesis, had an ASPECT score of 7 on brain computerised tomography (CT) (A). Left M1 occlusion is detected in brain CT angiography (B), partial recanalization is achieved after mechanical thrombectomy (C). Luxury perfusion is observed on CT taken 24 hours later when the NIHSS score is 6 (D). Reperfusion edema is observed in the CT taken 7 days later when the clinical condition worsens (E) and decompressive craniectomy is performed (F).

related to ischemic core volume (137). Proximal cerebral artery occlusions often result in large penumbra areas that are vulnerable to reperfusion injury following revascularization. As the therapeutic treatment window extends beyond 6 hours in patients with appropriate perfusion imaging, longer periods of hypoperfusion occurring in these areas increase the susceptibility of salvaged tissue to additional injury.

Early recognition and control of hypertension is the most important factor in the prevention of reperfusion-induced edema. This is important even in patients with a normotensive course. Preoperative and postoperative use of Transcranial Doppler ultrasonography in carotid revascularization may help identify patients with increased cerebral blood flow and associated hyperperfusion risk. Blood pressure should be lowered with antihypertensives that do not increase CBF and do not cause excessive vasodilation (138).

Further investigation of the multi-factorial mechanisms of reperfusion edema is only the beginning of significantly improving stroke management (130).

\subsection{Perihematomal Edema in Intracerebral Hemorrhages}

Intracerebral hemorrhage constitutes 10$15 \%$ of all strokes, and its incidence is gradually increasing due to the aging population and the widespread use of antithrombotic agents. The high mortality and long-term disability risk and the lack of a specific treatment make intracerebral hemorrhage a significant public health problem (139).

Turkish Journal of Cerebrovascular Diseases 2021; 27(2): 65-132 
Regardless of the etiology, the mechanical effect caused by acute hematoma is responsible for primary cerebral damage, and in some cases, the increase in hematoma volume observed in the early period worsens the prognosis. The edema that starts to form around the bleeding area from the first hours of intracerebral hemorrhage is called perihematomal edema (PHE) and is considered an important cause of secondary cerebral damage. Although the rate of increase in PHE volume is highest in the first 24 hours and slows down after 48 to 72 hours of bleeding, the expansion continues and PHE reaches its maximum size in an average of two weeks. PHE can cause a mass effect and herniation, eventually leading to neurological deterioration. Despite medical applications and advanced surgical techniques for the primary damage caused by hematoma in the intracerebral hemorrhage treatment, the desired improvement in the clinical outcome could not be achieved, and PHE has become one of the treatment targets.

More than one mechanism is thought to be involved together in the development of PHE. The process, which begins with the passage of fluid from the vascular bed to the perihematomal area due to the osmotic gradient that occurs following bleeding, continues with blood-brain barrier damage, complement activation and inflammatory response, especially induced by thrombin. In the early phase, cytotoxic edema is also considered to contribute to PHE. Moreover, hemoglobin degradation products accumulating after lysis of erythrocytes during hematoma resolution play a role in the pathogenesis of PHE by causing free radical damage and neuron death $(140,141)$.

PHE can be considered a neuroradiological marker of secondary brain injury. While hypodensity surrounding the bleeding area in computed tomography suggests PHE development, it appears as increased perihematomal signal in T2-weighted MRI sequences. MRI is seen as a superior method in PHE measurement, as it can be confused with ischemic foci in neighboring regions depending on the localization of bleeding in tomography (142) (Image 4).

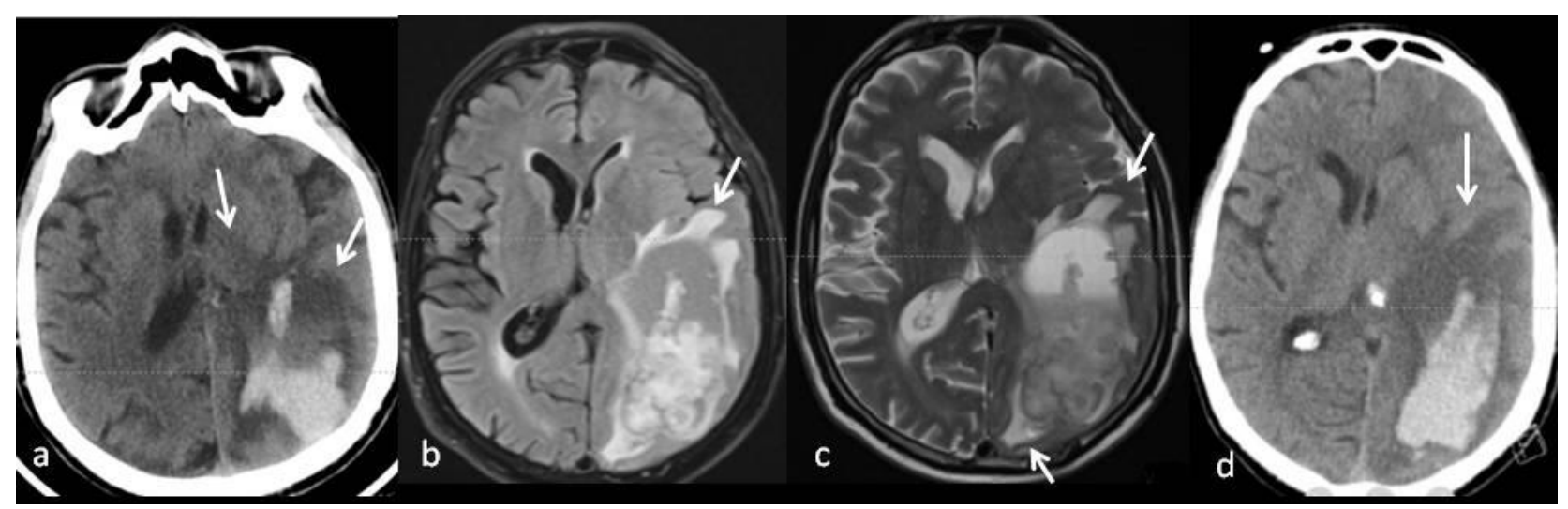

Image 4. Acute ICH and PHE detected on the initial CT of the patient presenting with a sudden loss of consciousness and right hemiparesis; in (a) FLAIR (b) and T2 (c) sequences, PHE is hyperintense around the hematoma; the 24-hour CT gained due to worsening of consciousness shows a compression effect on the midline structures (d).

PHE, which is observed in most intracerebral hemorrhage cases, is affected by many factors. Studies have revealed a significant correlation between PHE and hematoma volumes detected at the time of admission to the hospital (143). In the light of this information, it can be inferred that medical and surgical interventions to be applied in the acute period may limit PHE development. Low PHE volume detected in warfarin-related intracerebral hemorrhages has been explained by the significance of coagulation cascade activation in edema development (144). Diabetic and hypertensive patients have been shown to have a higher risk of PHE, $(145,146)$ but the evidence that aggressive blood pressure management slows PHE development could not be supported by repeated study results (147). Statin use before intracerebral hemorrhage was associated with a lower risk of PHE (148).

With secondary brain injury becoming a potential treatment target, research on the relationship between PHE and prognosis has intensified. One of the parameters evaluated in studies, 'PHE expansion rate', stands out in 
showing a significant relationship with poor 90day functional outcome. However, current data are still insufficient to establish a clear relationship between PHE and prognosis, and further studies are needed to determine the most appropriate parameters for measurement (149).

The use of hyperosmolar agents and surgical interventions are the standard approaches applied for the mass effect of PHE. Treatments targeting complex cellular and molecular mechanisms are still under investigation. In recent years, preclinical studies have shown that especially complement and thrombin inhibition and iron chelation treatments provide a decrease in edema formation and neurological improvement in experimental intracerebral hemorrhage models (140).

\subsection{Herniation Syndromes in Stroke: Types and Clinical Findings}

A brain herniation is the displacement of brain tissue from one compartment to another. It is a life-threatening condition leading to respiratory arrest and needs urgent diagnosis. Brain herniation can compress cranial nerves and vessels, causing hemorrhage, ischemia, or hydrocephalus (150). The increase in intracranial pressure is somewhat limited by compensatory displacement of the cerebrospinal fluid and changes in cerebral blood volume, as specified in the Monro-Kellie doctrine (151). If the compensatory mechanisms fail, the brain herniates from the different compartments formed by the dural septum (Figure 7).
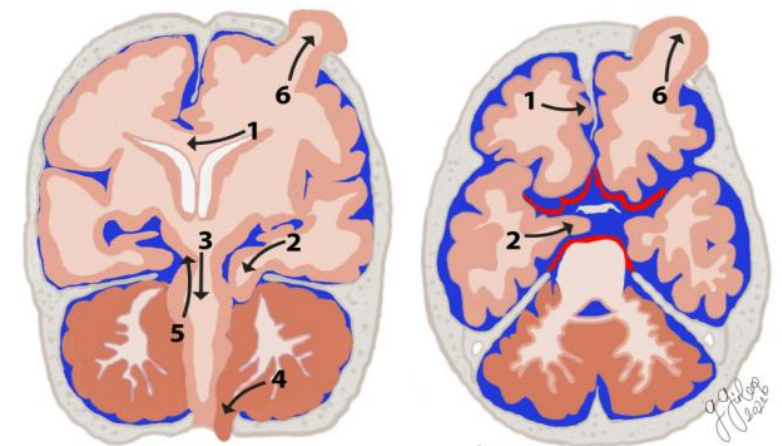

Figure 7. 1. Subfalcine 2. Lateral Descending Transtentorial (Uncal) 3. Central Descending Transtentorial 4. Tonsillar 5. Ascending transtentorial 6. External herniation (compiled from Johnson) (150).

Many diseases such as tumors, hemorrhages, malignant cerebral infarcts, hydrocephalus, and infections may predispose to brain herniation. Malign middle cerebral artery infarction occurs when more than two-thirds of the middle cerebral artery territory is affected, usually leading to acute cerebral edema, increased intracranial pressure, and clinical progression to brain herniation in the first 48 hours. It is used to describe MCA occlusions that can cause transtentorial herniation and death due to mass effect, usually within the first week despite aggressive medical therapy (5, 152).

Brain herniation can be divided into two main categories; intracranial and extracranial herniations. There are 3 subtypes of intracranial herniation; $1 . \quad$ Subfalcine herniation, 2. Transtentorial herniation, 3. Tonsillar herniation (Figure 7). Extracranial herniation refers to the protrusion of the brain tissue from the skull fracture and can also occur iatrogenic after craniotomy (153).

1. Subfalcine herniation: Also known as midline shift or cingulate herniation, it is the most common type of herniation. The ipsilateral cingulate gyrus herniates under the anterior falx, causing infarction in the distal region of the anterior cerebral artery. The posterior falx is more rigid and more pressure-resistant than the anterior (154). Compression of the anterior cerebral artery, especially the pericallosal and callosomarginal arteries, causes ischemia. Brain edema resulting from ischemia further increases the mass effect. Depending on the infarction of the motor homunculus, unilateral or bilateral lower extremity weakness, incontinence and coma may be observed. The degree of midline shift is related to prognosis; shifts less than $5 \mathrm{~mm}$ have a good prognosis, whereas shifts more than $15 \mathrm{~mm}$ are associated with a poor outcome (155). Subfalcine herniation are best visualized on coronal $M$ sections.

2. Transtentorial herniation: It may occur with expanding mass lesions (e.g., intracerebral, subdural, or epidural hemorrhage, malignant infarction, abscess, tumor, obstructive hydrocephalus). In this type of herniation, the impairment of consciousness is usually related to the lateral displacement of the midline structures rather than downward herniation. In particular, a shift of the pineal gland larger than $8 \mathrm{~mm}$ is associated with consciousness disorders; if the shift is more than $11 \mathrm{~mm}$, coma develops (156). Symptoms of papilledema and Cushing's triad 
(hypertension, bradycardia, irregular breathing) may be seen due to increased intracranial pressure. Transtentorial herniation can be seen in two main types as descending and ascending (154).

a) Descending Transtentorial Herniation: It occurs when the brain tissue is displaced downward through the tentorial notch. It is divided into two subtypes, lateral and central.

Lateral herniation has two subtypes as anterior and posterior. It occurs as a result of displacement of the medial temporal lobe.

The anterior subtype is also known as uncal herniation. It is the best-understood type of herniation, and the uncus herniates into the ipsilateral crural cistern. The earliest finding in uncal herniation is effacement of the suprasellar cistern. Classic clinical findings are large pupil (85\% ipsilateral) unresponsive to light, contralateral hemiplegia, and coma. As the herniated segment is displaced further, shifting causes compression of the contralateral cerebral peduncle in the Kernohan space, damaging the contralateral corticospinal tract, consequently causing ipsilateral paresis or paralysis on the side of the lesion. This clinical picture is known as the "Kernohan phenomenon" (false localization sign) (156).

In the posterior subtype, the parahippocampal gyrus is displaced towards the posterolateral part of the tentorial incisura (150).

In central hernias, diencephalon, mesencephalon, and pons herniate. Due to the compression of the penetrating arteries feeding the diencephalon, ischemia develops in the ascending reticular activation system, thereby impairing wakefulness. Progressive central herniation can lead to oculomotor paralysis, progressive altered consciousness, decerebrate posture, change in breathing pattern (such as Cheyne Stokes respiration, central neurogenic hyperventilation, and apneustic breathing, respectively), coma, and eventually death (150). If the pituitary gland is affected, diabetes insipidus develops. It causes a progressive clinical picture with a worse prognosis than uncal herniation (157).

b) Ascending Transtentorial Herniation: If the brain stem herniates upward from the tentorial space, the dorsal mesencephalon, aquaductus cerebri, and arteries are suppressed. This type of herniation develops in massive cerebellar infarcts, as in lesions of the cerebellar vermis when the space-occupying lesion is close to the incisura $(154,158)$. The other possible cause is the sudden reduction of supratentorial intracranial hypertension (159). Depending on the size of the tentorial incisura, the herniation pattern may change. When the tentorial opening is large, the cerebellar vermis upwards; if it is small, the cerebellar tissue will herniate down the foramen magnum (tonsillar herniation) $(154,158)$. Clinical findings include acute hydrocephalus, Parinaud's syndrome, and limitation of upward gaze.

3. Tonsillar herniation: It occurs as a result of the cerebellar tonsils shifting into the foramen magnum due to pressure and compressing the bulb. An acute increase in intracranial pressure develops due to obstruction of the fourth ventricle. As a result of the bulbs being affected, acute hypotension may be observed in the patient, and breathing may suddenly stop $(151,154,159)$. Normal tonsil position relative to the foramen magnum varies with age. Mikulis et al. described the normal position of the tonsils below the foramen magnum in different age groups. In the first decade, the cerebellar tonsils $6 \mathrm{~mm}$ below the foramen magnum are considered abnormal. In the second decade, the reference value is $5 \mathrm{~mm}$; for the 4 th to 8 th decades, the threshold is greater than $4 \mathrm{~mm}$; and at age 80 years or older, $3 \mathrm{~mm}$ is the limit $(160,161)$.

4. Transalar herniation: It is a less defined form of herniation. It is also known as transsphenoidal herniation. It is usually associated with subfalcine and transtentorial herniations. In the descending type, the frontal lobe is displaced posteriorly and inferiorly over the sphenoid wing. It occurs in frontal space-occupying lesions. The middle cerebral artery can become occluded between the frontal lobe and the sphenoid ridge.

Ascending transalar herniation is the displacement of the temporal lobe anteriorly and above the sphenoid ridge due to a space-occupying lesion in the middle cranial fossa. The supraclinoid portion of the internal carotid artery may be compressed between the anterior clinoid process and the brain. It can be identified with posterior or anterior displacement of the middle cerebral artery in massive infarcts involving the temporal lobe (154). In conclusion, identifying the type of herniation and associated complications helps to decide on treatment and emergent surgical intervention. 
A definite prediction of whether intracranial edema that will progress to herniation will develop at the patient's first visit is not possible. However, the prognosis of the patient can be predicted with a high probability from the patient's characteristics, imaging, and clinical characteristics. Risk factors for the development of malignant edema after malignant MCA infarction are (162-167):

$\checkmark$ Onset of nausea-vomiting within 24 hours from symptom onset

$\checkmark$ Systolic blood pressure >180 mmHg 12 hours after onset

$\checkmark$ History of hypertension, heart failure, atrial fibrillation

$\checkmark$ Young age (since there is no atrophy in the brain tissue, it will be affected more quickly from the pressure increase)

$\checkmark$ Increased leukocyte

$\checkmark$ Admission NIHSS (National Institutes of Health Stroke Scale); $>20$ in the dominant hemisphere and $>15$ in the non-dominant hemisphere

$\checkmark>50 \%$ hypodensity in the MCA territory in the first 6 hours on brain CT

$\checkmark \quad$ Displacement of the septum pellucidum $>5$ $\mathrm{mm}$ from the midline to the opposite side on CT

$\checkmark$ Admission ASPECT (Alberta Stroke Program Early CT) score $<8$

$\checkmark$ Internal carotid artery occlusion in CT angiography, poor collateral circulation

$\checkmark \quad$ Infarct volume $>145 \mathrm{ml}$ in brain magnetic resonance (MR) imaging

$\checkmark$ Increased pulsatility index in Transcranial Doppler

$\checkmark \quad$ Increase in optic nerve sheath diameter on imaging
Many scoring systems (Kasner risk index, EDEMA score, MBE score, DASH score) have also been developed to predict the development of malignant cerebral edema after hemispheric stroke (165, 167-169). However, all these predictive parameters are beneficial only in guiding the clinician, and it is not appropriate to evaluate the patient solely based on these parameters. The patient should be followed very closely clinically and radiologically for brain edema from the first admission. Worsening of consciousness, drowsiness, vomiting, and a newly developing neurological deficit in a patient who was awake at admission should warn the clinician for the development of brain edema. These patients should be followed in centers with neurology intensive care units and neurosurgeons for a possible surgical requirement.

3.2 Herniation Syndromes in Ischemia: CT Findings

Herniation types and brain CT samples in cerebral ischemia are shown (Image 5-11) (170).

1-) Subfalcine (Singular) Herniation

2-) Transtentorial Herniation

a) Descending Transtentorial Herniation

- Temporal (Uncal) Herniation

- Central Herniation

b) Ascending Transtentorial Herniation

3-) Tonsillar (Downward cerebellar Transforaminal) Herniation

4-) Transalar (Transsphenoidal) Herniation Descending Transalar (Transsphenoidal) Herniation

b) Ascending Transalar

(Transsphenoidal) Herniation

5-) Extracranial (Transcalvarial) Herniation

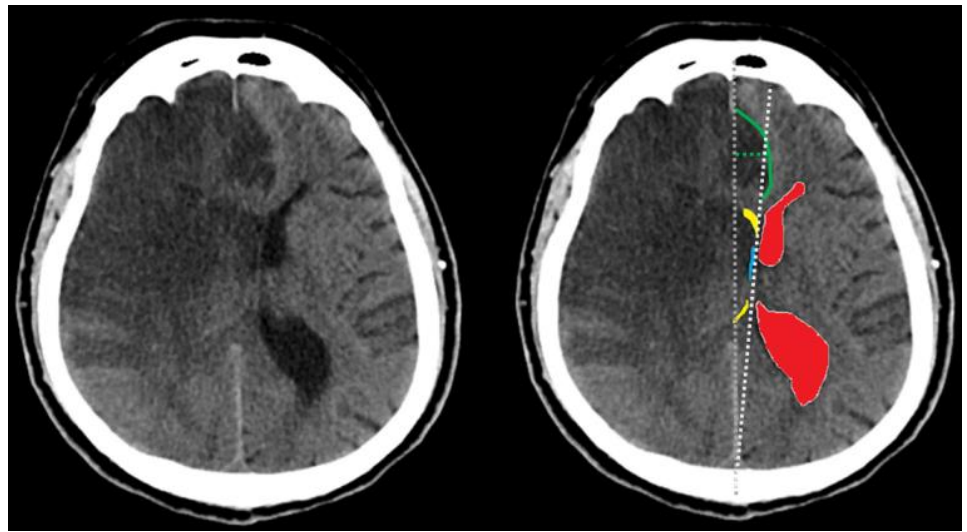

Image 5. Subfalsin (Singuler) Herniasyon. Non-enhanced brain CT axial sections show a shift of the cingulate gyrus (green line), ipsilateral ventricular compression (yellow area) \& partial collapse of the foramen of Monro (blue line), causing contralateral ventricular dilatation (red area). 

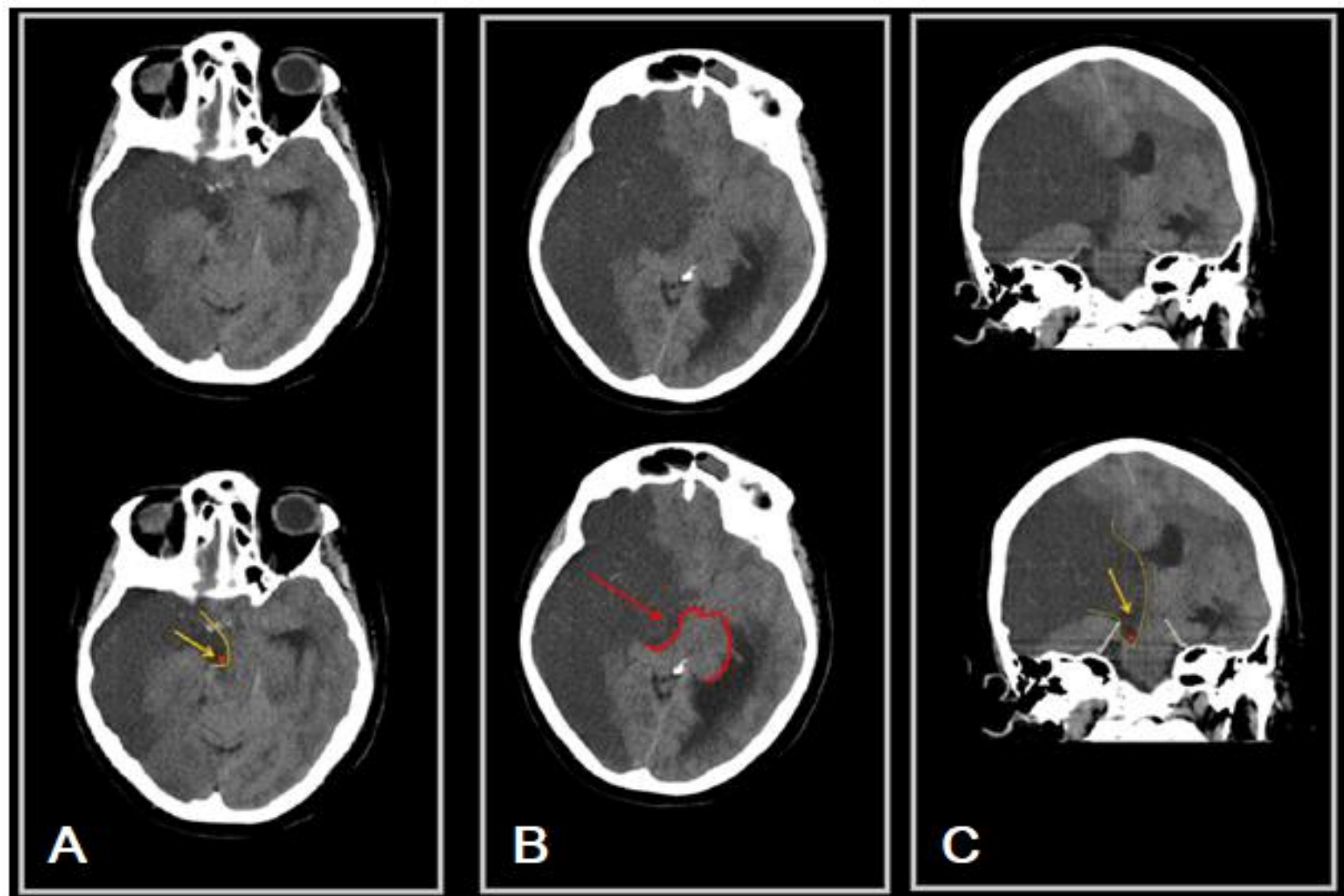

Image 6. Temporal (Uncal) Herniation. Brain CT shows uncus (red dot) herniation (yellow arrow) on axial (A) and coronal (C) sections and a shift in the mesencephalon secondary to herniation on axial section (B).

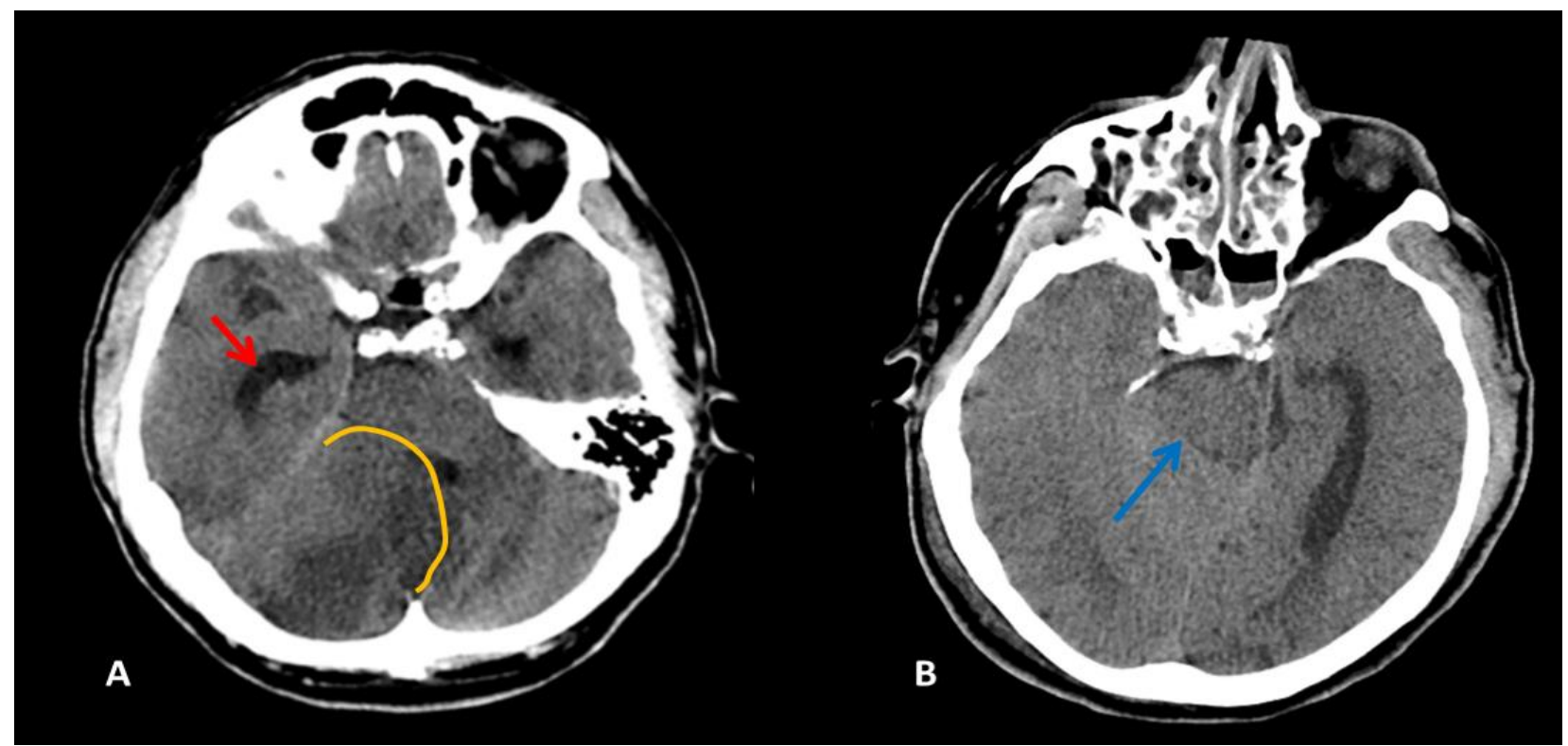

Image 7. Ascending Transtentorial Herniation. Non-enhanced brain CT axial sections show (A) dilatation compatible with hydrocephalus in the right lateral ventricular temporal horn (red arrow), compression of the fourth ventricle (yellow line) due to the mass effect of edema secondary to right cerebellar ischemia, and (B) obliteration of the right quadrigeminal cistern (blue arrow).

Turkish Journal of Cerebrovascular Diseases 2021; 27(2): 65-132 


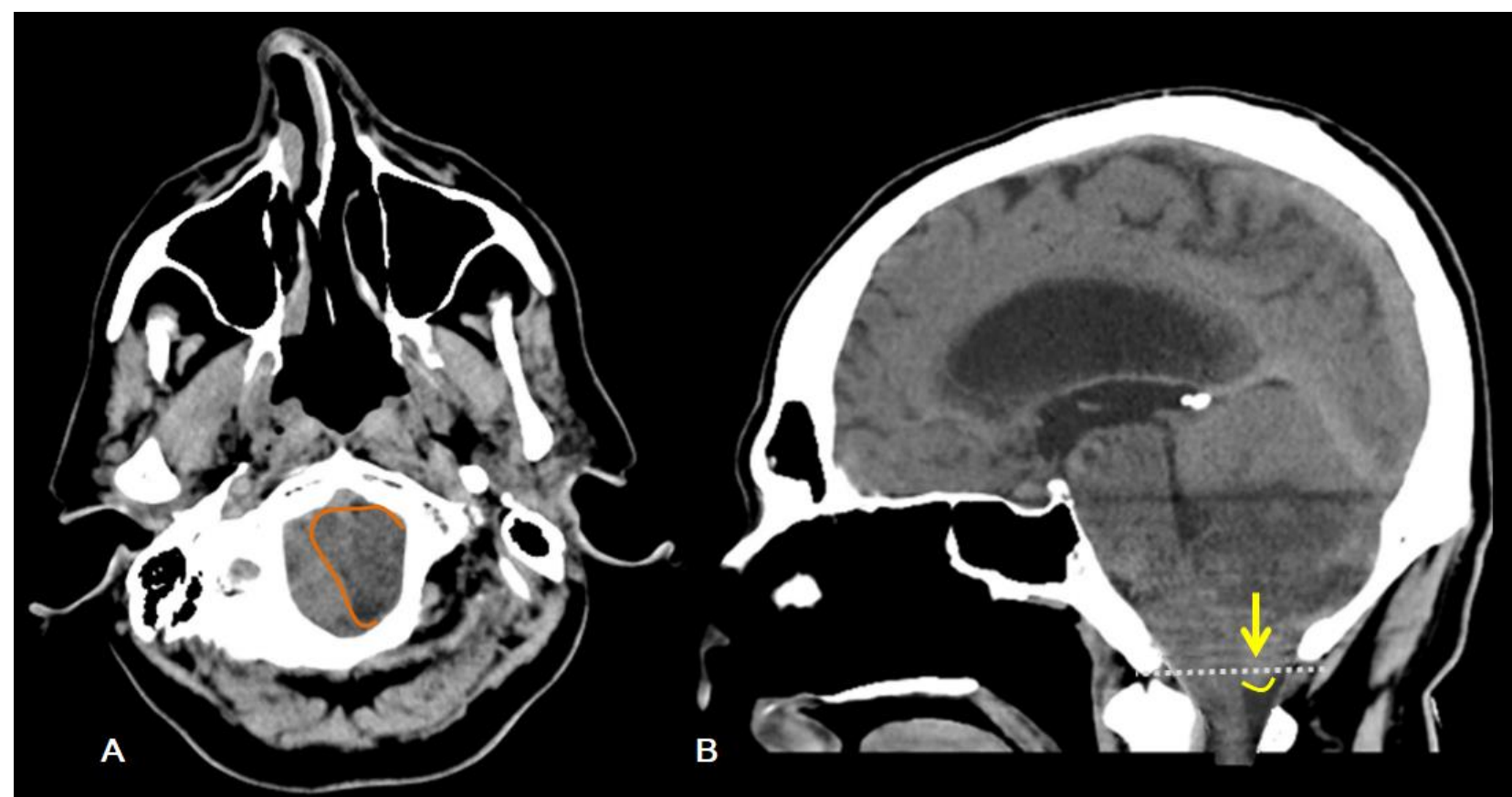

Image 8. Tonsillar (Downward cerebellar - Transforaminal) Herniation. Non-enhanced brain CT shows herniation (yellow arrow) below the foramen magnum (dotted line) of the left cerebellar tonsillar shift (orange line) in the axial section (A) and the left cerebellar tonsillar (yellow line) in the sagittal section (B).

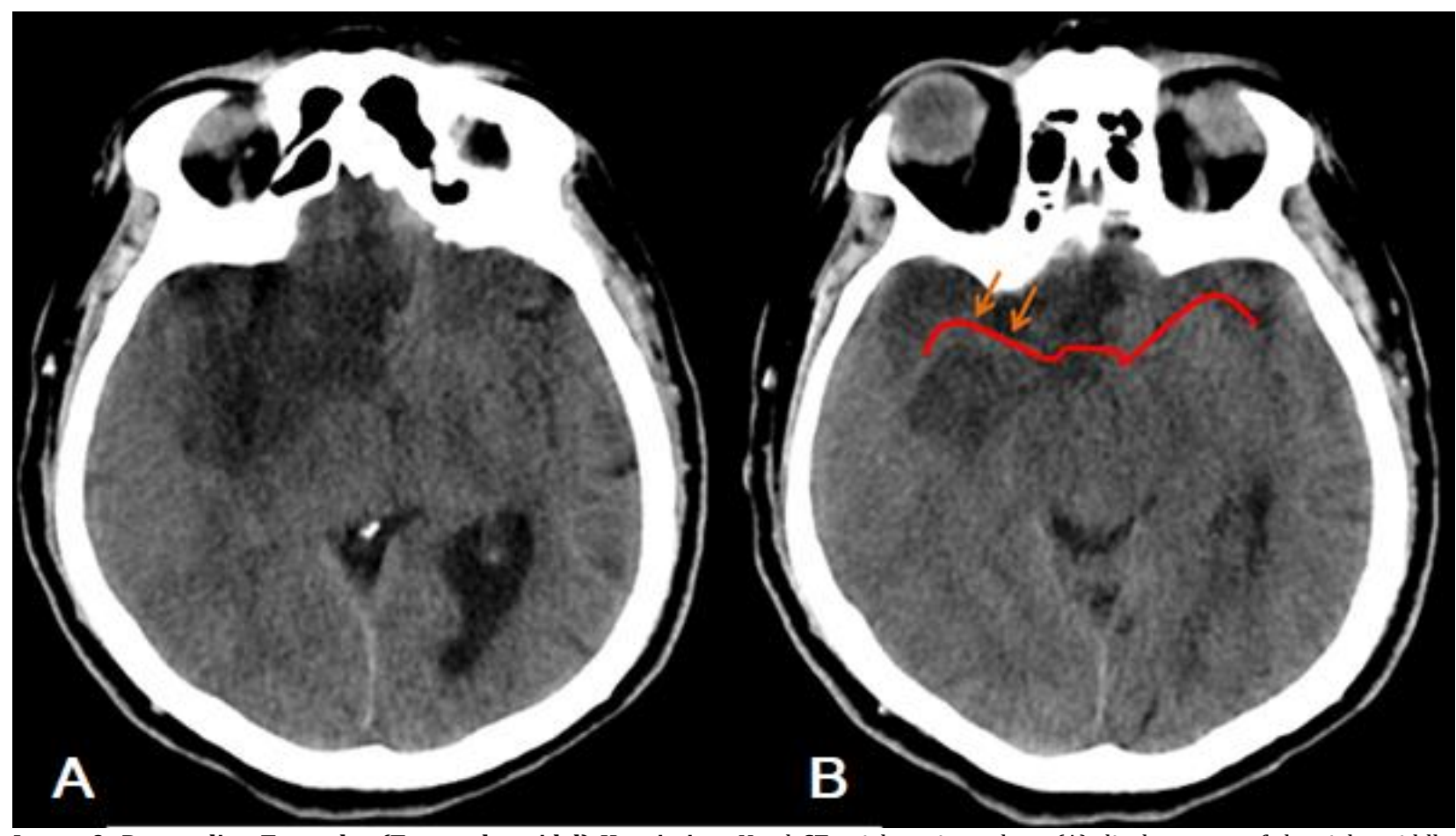

Image 9: Descending Transalar (Transsphenoidal) Herniation. Head CT axial sections show (A) displacement of the right middle cerebral artery and frontal lobe orbital surface towards the posterior and inferior on the sphenoid wing due to the mass effect of edema caused by the right frontal lobe ischemia (B) (orange arrows). 


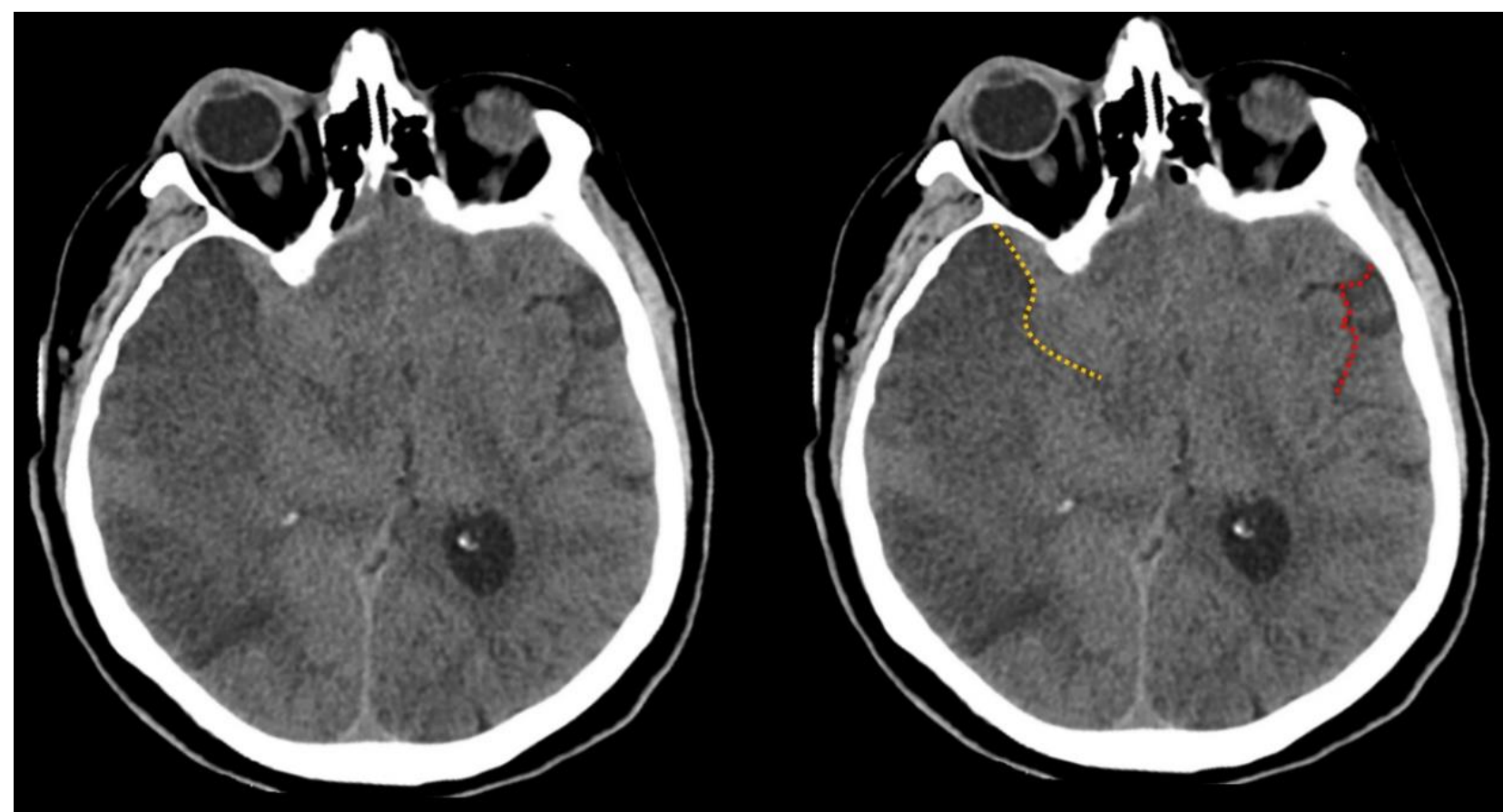

Image 10. Ascending Transalar (Transsphenoidal) Herniation. Cranial CT scan, axial sections reveal that the right Sylvian fissure (and the associated middle cerebral artery) is displaced anteriorly (yellow line) due to the mass effect of edema caused by the right temporal lobe ischemia in the middle cranial fossa, while the Sylvian fissure is in its normal position on the left (red line).

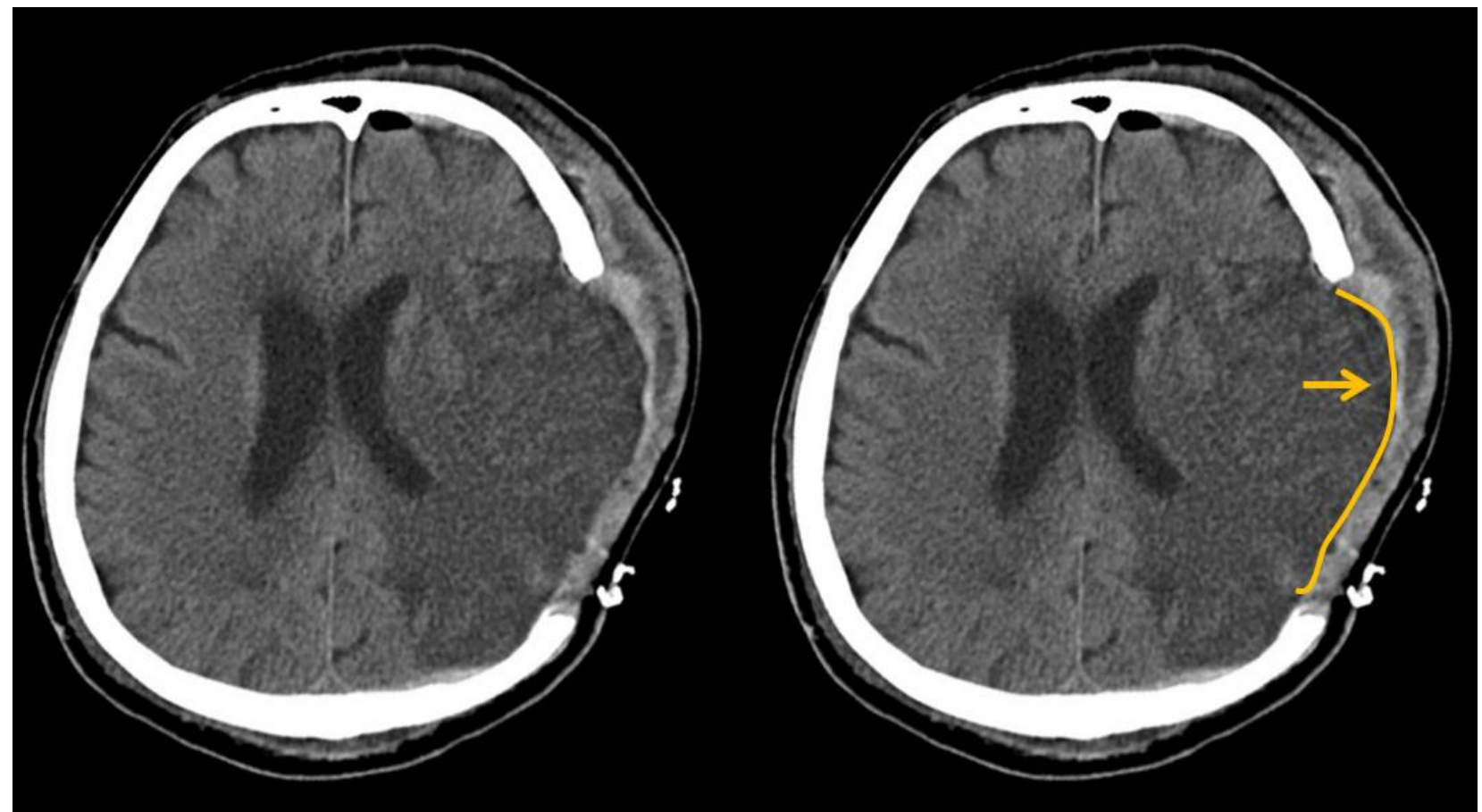

Image 11. Extracranial (Transcalvarial) Herniation. Non-enhanced brain CT axial sections show extracranial herniation of the left hemisphere after craniectomy (yellow arrow and line).

Turkish Journal of Cerebrovascular Diseases 2021; 27(2): 65-132 


\section{Types}

3.3 Radiological Diagnosis of Brain Edema

Cerebral edema, which is caused by the abnormal movement of water between the compartments in the brain tissue, can be detected from the early period by imaging methods. Indeed, our radiological information about ischemic tissue in the very early period after stroke is based on our ability to follow this water flow. We can predict excessive brain edema, which causes increased mortality rates in the first days after stroke, before it occurs, by looking at some radiological imaging findings at admission.

Cytotoxic edema, which is seen in the early period of ischemic stroke, especially in the first 24 hours when life-threatening edema is not expected, is also known as cellular edema and occurs when extracellular water enters the cell. Although mainly the gray matter is affected, changes can also occur in the white matter. At this stage, the blood-brain barrier is still intact. The location of occlusion in post-contrast angiography can be determined in computed tomography (CT), the most widely used diagnostic tool after stroke, and the areas of reduced blood flow can be detected in CT perfusion, whereas in non-contrast brain CT, changes in cytotoxic edema in the parenchyma can be identified in the early period only in $1 / 3$ of the cases. In other words, early noncontrast brain CT examination is not sufficient to show parenchymal findings of cytotoxic edema. Findings that can be detected in very few cases are; fading of the lentiform nucleus borders, the disappearance of the insular rib, sulcal effacement, and early hypodensity, which are known as early signs of ischemia (Image 12). In this period, ischemic tissue size can be inferred by evaluating the magnitude of hypodensity in the ischemic area and calculating the ASPECT score.

The modality best visualizing the changes of cytotoxic edema is magnetic resonance imaging (MRI). The sequence in which the findings of cytotoxic edema are best observed in the early period is diffusion-weighted examination (Image 13). Although no obvious pathology is observed in the parenchyma in T1, T2, and FLAIR in the early period in brain MRI, the MRI sequences used in diffusion imaging, which is sensitive to water diffusion, can visualize cytotoxic edema. The development of vasogenic edema in the brain tissue begins 4-6 hours after stroke symptoms and

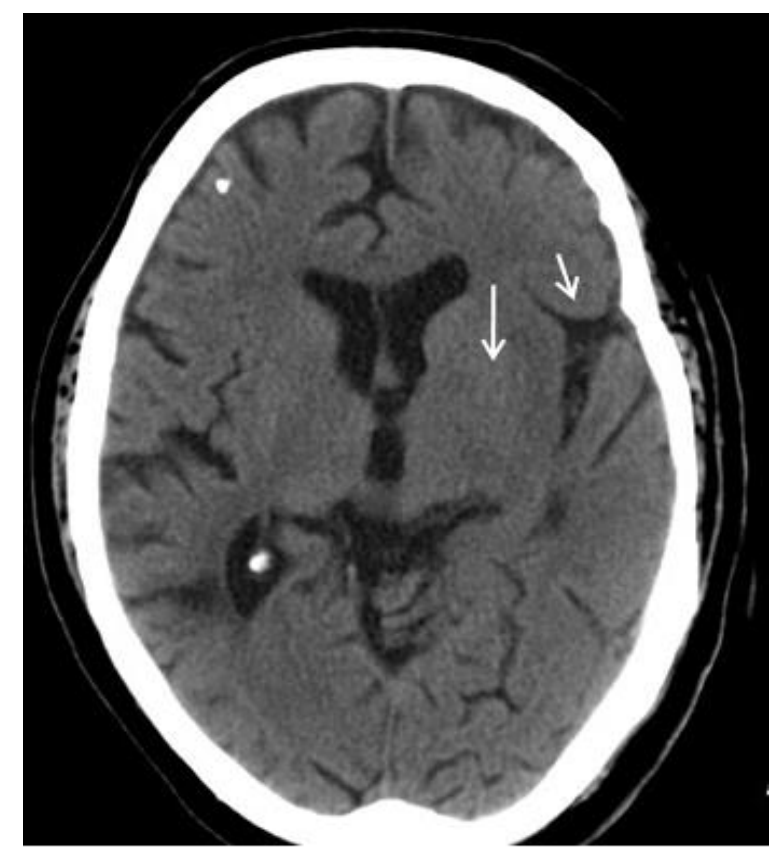

Image 12. In the axial CT examination, the gray-white matter differentiation in the left insular cortex (long arrow) is lost, and the left lentiform nucleus borders (short arrow) are not well observed. Image of an incidentally observed old infarct in the posterior perirolandic area in the right parietal region in the same case. (Courtesy of Dr. Nafisa Shakir Batta Radiopaedia.org, rID: 26343)

peaks at 24-48 hours. Vasogenic cerebral edema secondary to the impaired blood-brain barrier occurs in the extracellular space and mainly affects the white matter. Apart from ischemia and cerebral hemorrhage in the brain, it can be observed around the lesion in pathological conditions such as brain tumor, abscess, and contusion.

Large hemispheric infarcts constitute 5-10\% of all ischemic strokes and the most severe forms of ischemic stroke. Large infarcts occur with total occlusion of the internal carotid artery or proximal middle cerebral artery, also known as malignant middle cerebral artery syndrome $(171,172)$. More than $40-50 \%$ of this type of large infarcts can cause malignant cerebral edema on the 2 nd to 3 rd day of symptom onset, and the clinical condition might worsen in the first week (173). Large hemispheric infarcts can be identified by the presence of hypodensity greater than $50 \%$ of the MCA area on brain CT, the presence of dense MCA, an ASPECT score less than 6, and a total ischemic volume greater than $145 \mathrm{~mL}$ in the diffusionweighted MRI at admission (174). 
Vasogenic edema can be observed in both white and gray matter. While T1-T2 changes are not expected on MRI in cytotoxic edema, vasogenic edema is hypointense on T1 and hyperintense on T2. Since vasogenic edema develops secondary to blood-brain barrier disruption in all structural lesions, it is not possible to radiologically distinguish between vasogenic edema seen after intracranial mass, PRES, abscess, meningitis, etc., and vasogenic edema due to ischemic stroke.

If cerebral edema following ischemic damage reaches a certain amount, it can cause a mass effect, increase the intracranial pressure over time, and cause the brain tissue to shift to the opposite side (Image 13). The patient's clinical condition and the control imaging are extremely important for deciding on the necessity of decompressive surgery during or before this period. The extent of ischemic damage and edema can lead to herniation, severe brain damage, and even death (Image 14). Sometimes, cerebral edema can cause a pseudo-SAH image by suppressing dural venous structures (175). To get the maximum benefit from radiological imaging, we must decide what kind of information we expect to obtain from which modality in which period. The first radiological findings of ischemia in the first hours of stroke occur due to brain edema. Attention should be paid to the presence of the abovementioned findings that may help predict the prognosis in the neuroimaging examination performed at admission.
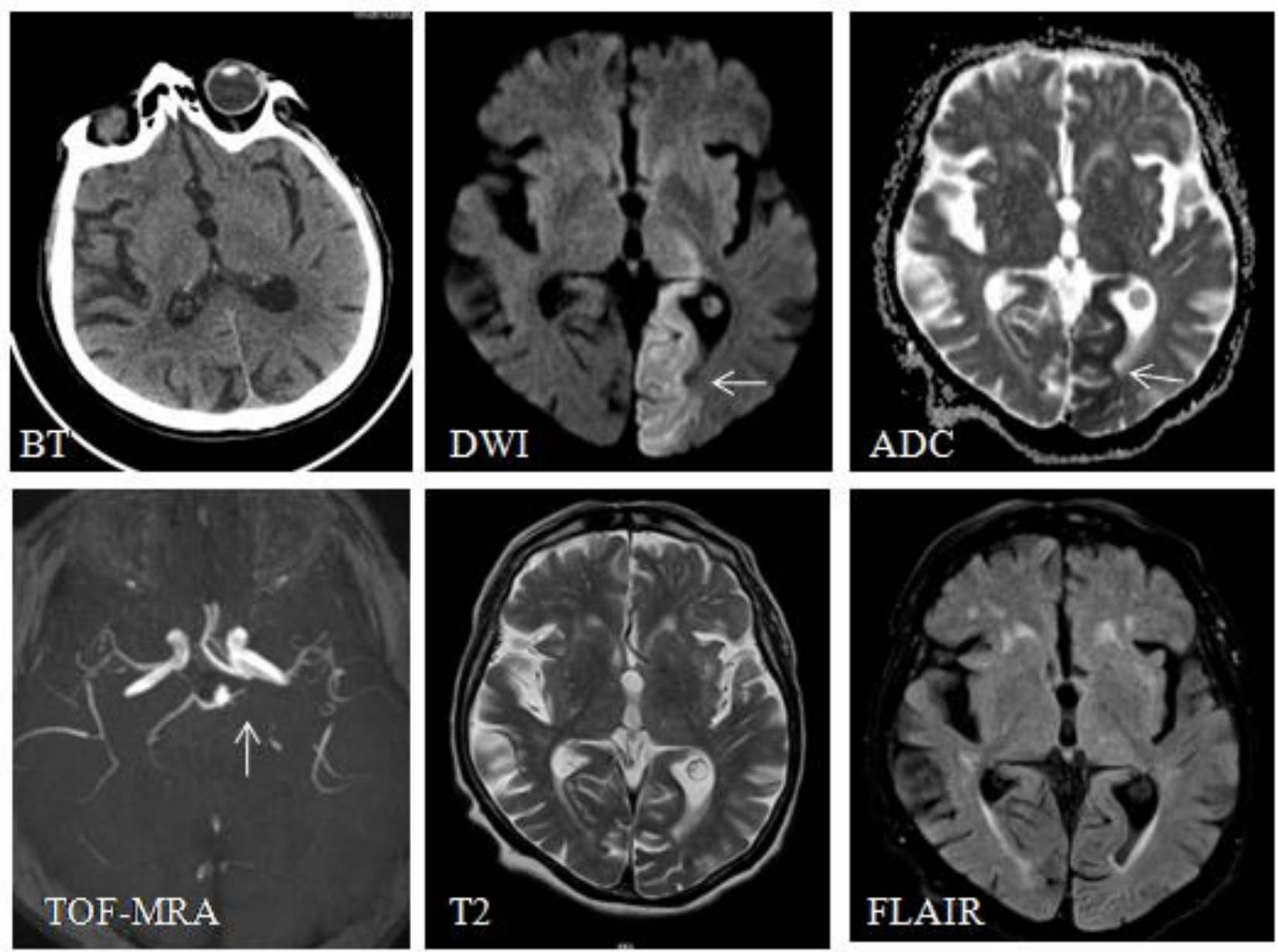

Image 13. A 95-year-old female patient presents at the 90th minute of symptom onset with loss of strength on the right side and right homonymous hemianopia. Although the cranial CT findings are normal, the left posterior cerebral artery area occluded in the TOF MRA is hyperintense on diffusion-weighted imaging and hypointense on ADC. In this period, no pathology is observed in T2 and FLAIR. 

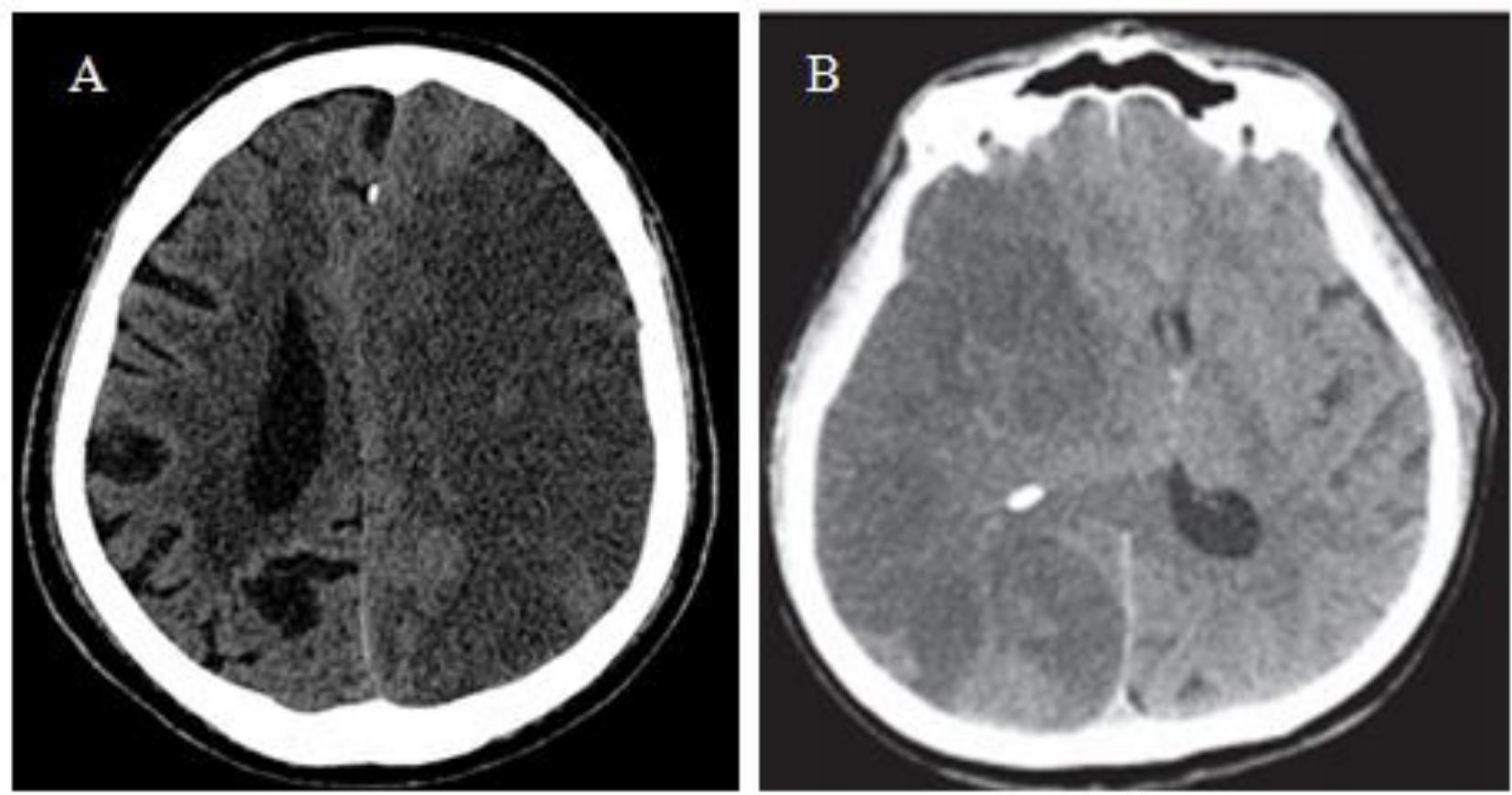

Image 14: Axial brain CT scan in the subacute period; a wide hypodense area and shift towards the opposite hemisphere in a 85 years old patient with left middle cerebral artery (A), and a 63 years old patient with right middle cerebral artery infarction and edema.

\subsubsection{Transcranial Doppler Sonography}

The introduction of Transcranial Doppler (TCD) into clinical use by Rune Aaslid in 1982 enabled sonographic examination of the basal cerebral arteries. It evaluates the basal cerebral arteries, using a low-frequency and usually $2 \mathrm{MHz}-$ frequency ultrasound probe, through areas with relatively thinner skull bones and flat tabula interna or an opening called the acoustic window (176). In TCD ultrasonography, the Doppler spectrum obtained with the principle of the Doppler effect is analyzed and interpreted. In spectrum analysis, along with values such as peak systolic velocity (Vs or Vmax), end-diastolic velocity (Vd), pulsatility index (PI), mean flow velocity (Vmean), the form of the Doppler spectrum is also evaluated (Image 15).

For continuous monitoring of intracranial pressure (ICP) in neurological intensive care units, invasive epidural, subdural, intraparenchymal, and intraventricular methods can be used, however, invasive monitoring may cause interventionrelated complications, such as bleeding, infection, and catheter slippage $(177,178)$.

The invasive method is a recommended follow-up method in advanced ICP treatment plans such as arterial blood pressure adjustment. TCD can be beneficial in ICP follow-up as a noninvasive, reproducible, and bedside method (179). Studies have shown specific patterns of TCD in patients with increased ICP, but most of these findings were from cases with severe head trauma, intracranial hemorrhage, brain tumor, hydrocephalus (180-182). A decrease in Vd in the TCD spectrum, a prominent systolic peak, and an increase in PI are observed in ICP increase, and additional information can be obtained from the TCD waveforms. TCD spectral changes in two patients with idiopathic intracranial hypertension (IIH) and high lumbar puncture opening pressure in our laboratory are shown in Image 16. Compared to the TCD spectra with normal ICP in Image 15 above, an increase in PI is observed along with a sharper decrease at the end-systolic and a decrease in diastolic.

TCD is also used as a supportive test in the diagnosis of brain death since it shows cerebral circulatory arrest that develops as a result of severely increased ICP. TCD examinations in brain death allow a more reliable determination of the spectral changes that develop with the increase in ICP (183). While a moderate increase in ICP causes the spectral changes shown in the figüre above 

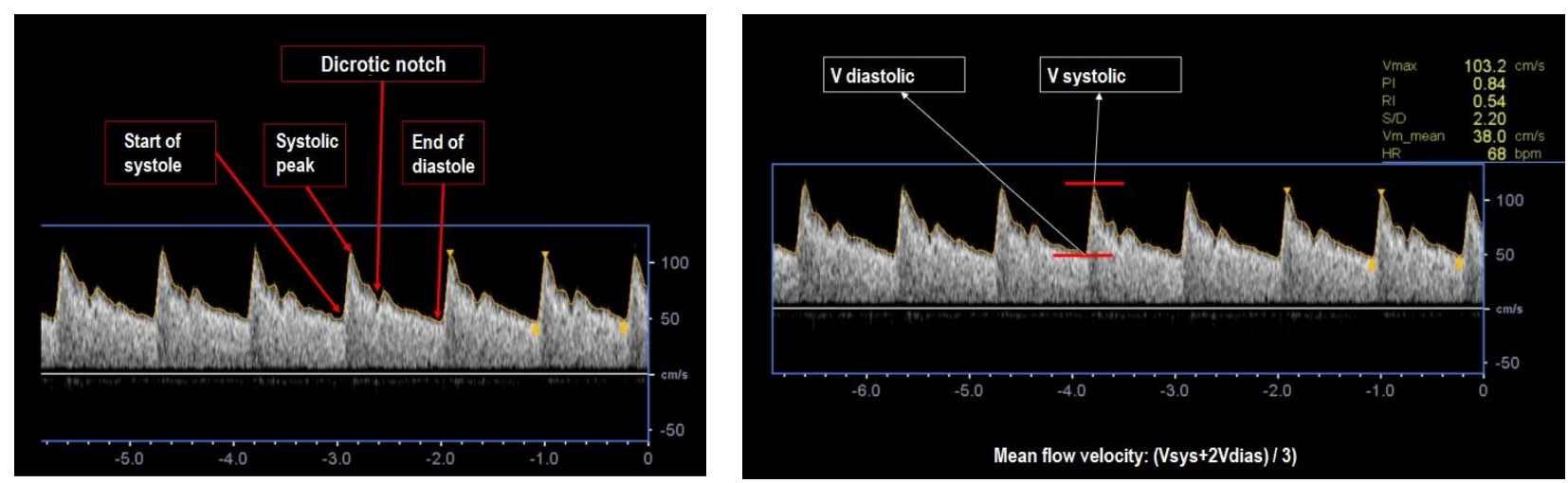

Image 15. Doppler spectral reflection of the cardiac cycle and calculation of flow velocities.
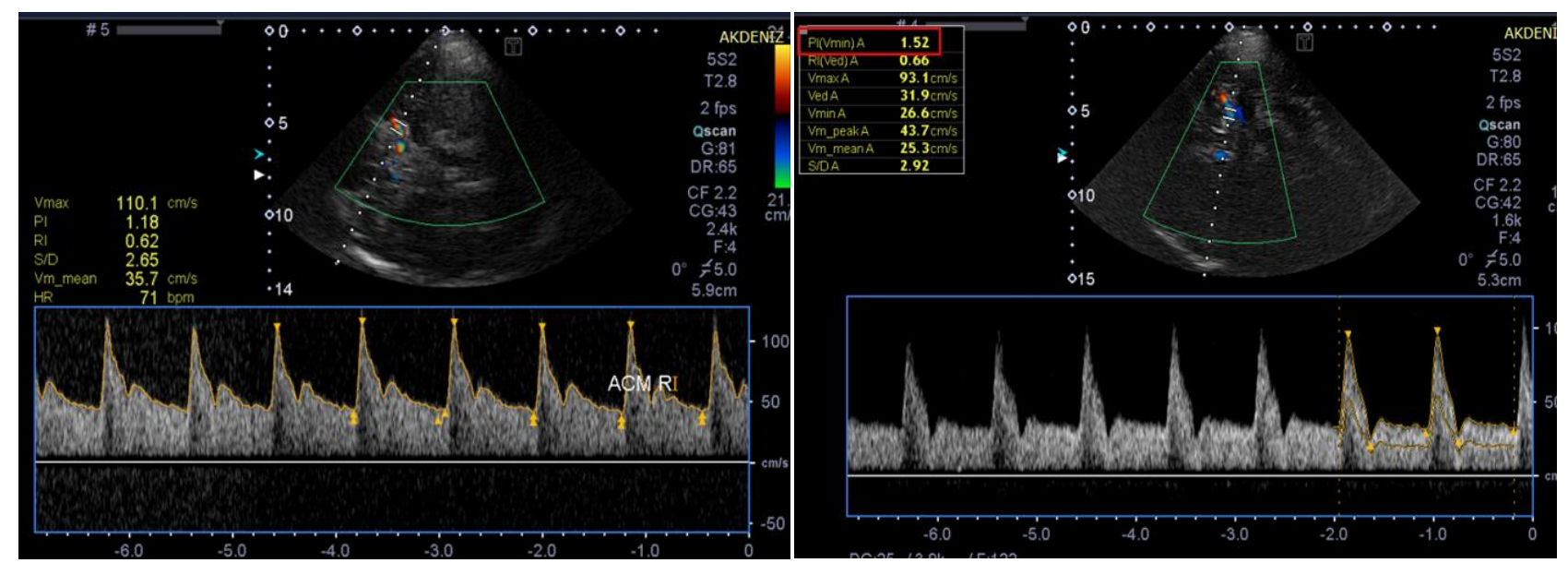

Image 16. TCD recordings of two patients with idiopathic intracranial hypertension with CSF pressures of 53 and $58 \mathrm{cmH} 20$, respectively.

(Image 16), diastole disappears or reverses in ICP increases that stop cerebral circulation (Image 17).

PI can be defined as a mathematical formula that aims to eliminate the angle dependence in Doppler measurements. The Gosling pulsatility index, the most commonly used hemodynamic index, describes the pulsatility of TCD waveforms. It is a ratio obtained by dividing the difference between systolic and diastolic flow velocity by the mean flow velocity and is formulated as $\left(\mathrm{V}_{\mathrm{s}}-\mathrm{V}_{\text {dia }}\right) /$ $\mathrm{V}_{\text {mean }}$ (184). PI, a parameter used to evaluate vascular resistance, is associated not only with cerebral vascular resistance but also cerebral perfusion pressure, arterial pressure pulse amplitude, compliance of the cerebral arterial bed, and heart rate. Studies mainly evaluating subarachnoid hemorrhage and head trauma etiologies, along with other etiologies, have shown that an increase in ICP is associated with an increase in PI. However, the use of PI alone in the determination of ICP increase is controversial due

Turkish Journal of Cerebrovascular Diseases 2021; 27(2): 65-132 to the effect of multiple physiological variables on this relationship (182, 185-189).

In ischemic stroke, TCD is used in many areas including diagnosis, etiology, and treatment. The use of TCD may be appropriate in the follow-up of post-infarction cerebral edema and subsequent ICP increase resulting from brain swelling. Especially in serial follow-up at the onset and after infarction, TCD can be used as a predictor of brain swelling and subsequent herniation, but further studies are required on this subject. A relationship was shown between the midline shift observed in brain imaging and the PI measured on the third day in patients whose PI was evaluated with TCD at regular intervals after middle cerebral artery infarction. Moreover, a PI above 1.5 was associated with a poor outcome (190). Another point to note is that TCD changes in the occluded artery occur not only due to rICP, but also due to conditions such as occlusion and stenosis in this artery. In a case report in which decompressive surgery was 


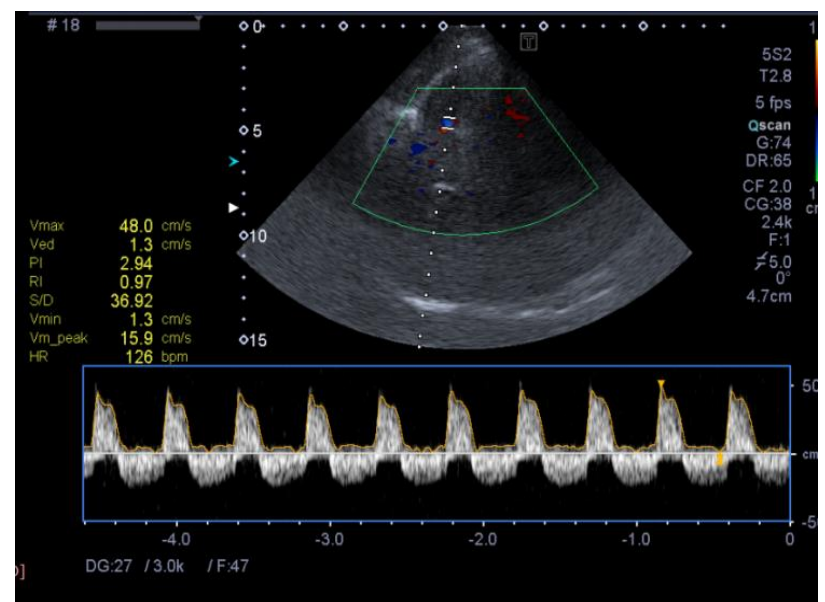

Image 17. Examples of TCD spectra findings in a brain death case.

performed due to middle cerebral artery occlusion, the increase in PI was found to be more pronounced on the occluded side before surgery. It has been reported that the increase in ICP becomes more pronounced, especially after the PI increase on the opposite side, and the findings improve on both sides after decompressive surgery (191).

There may be some limiting factors in the use of TCD in cases of cerebral infarct, associated brain edema, and ICP increase. Congestion in the affected artery, spectral form, and velocity changes caused by stenosis or partial recanalization will make it more difficult to interpret the findings. In addition, TCD changes in intracranial arteries caused by cervical artery stenosis and occlusion, two etiologies of infarct, also make it difficult to interpret. For instance, a hemodynamically significant internal carotid artery stenosis, which reduces systolic acceleration and Vs, may lead to misinterpretations during spectrum form evaluation and PI calculation. When evaluating the increase of the ICP after cerebral infarction, decisions should be made not only with TCD findings but with the help of medical examination and other inspections. Rather than the crosssectional evaluation of the TCD findings of ICP increase, assessment of changes occurring in the series follow-up and use of brain imaging methods accordingly will be a more appropriate approach.

\subsubsection{Transcranial B-Mode Sonography}

It should be noted that the triplex application of transcranial B-mode sonography can document both sonographic configuration changes such as

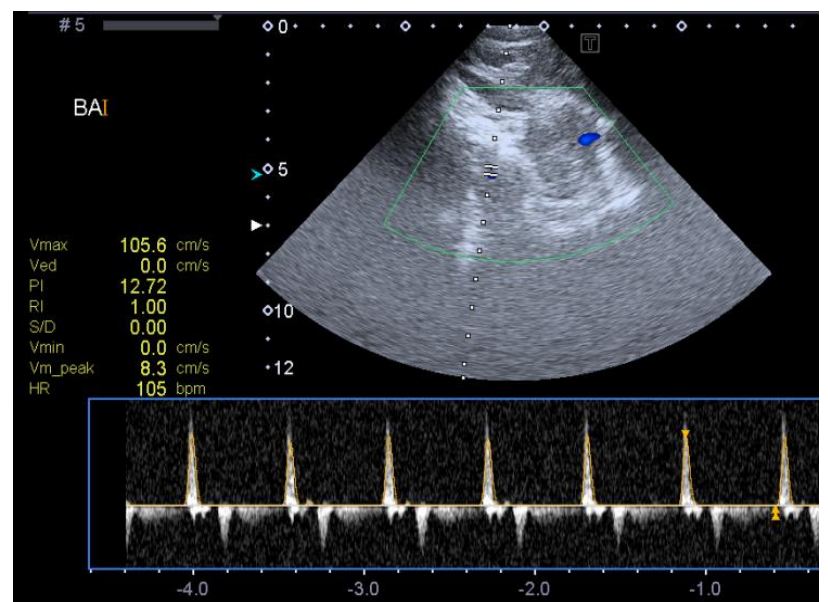

increased pulsatility due to ICP, as discussed above, and color Doppler changes that may indicate critical intracranial pressure increase $(183,192,193)$. Nevertheless, B-mode sonography stands out especially by allowing the midline shift (subfalcine herniation) to be determined numerically and followed at the bedside (192).

For midline shift detection, the distance from the transtemporal sonic window on both sides to the midpoint of the third ventricle and the outer edge of the cranium is measured. The midline shift is calculated as half the difference between the measurements made on both sides $(192,194)$. Sonography can be beneficial in evaluating the timing, titration, and efficacy of treatments such as osmotherapy and craniectomy, as it provides the opportunity to monitor the degree of midline shift, especially in malignant acute middle cerebral artery infarctions. The correlation between the shift measured by ultrasonography and computed tomography is over $90 \%$, sufficiently reliable and reproducible (194).

\subsubsection{Optic Nerve Sheath Diameter Measurement}

The optic nerve is a continuation of the brain, the optic nerve sheath is a continuation of the dura mater, and the subarachnoid space containing CSF continues between the optic nerve and its sheath. Pressure changes are also reflected in the preoptic area, thanks to the CSF circulation between the intracranial and intraorbital subarachnoid spaces (195). Due to the anatomical structure of the optic nerve sheath, the increase in CSF pressure causes the optic nerve sheath diameter to expand, 
especially in the anterior retrobulbar compartment (196). Optic nerve sheath diameter (ONSD) measurement provides an indirect assessment of intracranial pressure (197). Several studies have shown the correlation of ONSD enlargement with increased intracranial pressure using CT, MRI, and ultrasonography (196). Among these, ultrasonographic ONSD measurement stands out as an accessible, reproducible, and safe bedside examination (198).

ONSD ultrasonography can be performed with most color ultrasound systems that contain a high frequency (7.5 MHz or higher) linear probe (199). Following the system settings (mechanical index and thermal index) to protect sensitive structures such as the lens, retina, and vitreous body, all parameters including depth and grayscale are adjusted specifically for the patient in order to obtain the best image $(199,200)$. A standardized technique is required to ensure the correct assessment of optic nerve sheath boundaries, repeated evaluations by the measurer, and consistency between measurements made by different individuals (199). It is applied after covering the upper eyelid with a thick layer of gel with the patient in the supine position, the head and upper body in a $20-30^{\circ}$ elevated position, and the eyes closed and looking forward. The transducer is placed on the temporal side of the eye (201). In the axial plane, the longitudinal optic nerve can be easily seen $3 \mathrm{~mm}$ behind the homogeneous anechoic globe. In ultrasonography, the ONSD and hypoechoic optic nerve fibers between the outer hyperechoic borders of the subarachnoid space are measured using an electronic caliper, and the average of three separate measurements is taken. Although there are no clear diagnostic criteria showing increased intracranial pressure for ultrasonographic measurement of ONSD, studies conducted in Caucasians have shown that values above $5 \mathrm{~mm}$ can indicate increased intracranial pressure. Ethnic differences may change this limit (195). For demonstrating increased intracranial pressure in ischemic stroke patients, sensitivity and specificity were determined as $98 \%$ and $82 \%$, respectively, for a threshold value of ONSD > $5 \mathrm{~mm}$ (202). Gökçen et al. found mean values of $6.3 \mathrm{~mm} \mathrm{(5.2-}$ 7.1 ) in total anterior circulation infarcts and 5.2 $\mathrm{mm}$ (5.0-5.8) in lacunar infarcts (197).

Most studies report high sensitivity and specificity for ultrasonographic ONSD measurement in stroke (197, 202-205). Different studies have shown that in intracerebral hemorrhages, median binocular ONSD measurements better reflect the increase in intracranial pressure compared to Doppler examinations of retinal vessels (204), and in cases where the bleeding volume is above $2.5 \mathrm{~cm}^{3}$ in the first 6 hours of the hyperacute phase, sonographic OSCT enlargement is more sensitive (205). The ONSD measurement is also significant in acute stroke patients receiving thrombolytic therapy, and the ONSD values exceed the threshold values with the development of malignant middle cerebral artery infarction, suggesting that it may be beneficial in distinguishing patients with malignant cerebral edema risk in 24 hours (203).

In stroke, ultrasonographic ONSD measurement is an easy, non-invasive, reproducible method for monitoring intracranial pressure increase and cerebral edema in neurology intensive care units. Thanks to the ease of application at the bedside, it is especially beneficial in malignant middle cerebral artery occlusions in early diagnosis to reduce cerebral edema and herniation-related mortality and morbidity (Image 18).

\subsection{Pupillometry and other Non-Invasive Methods in the diagnosis of rICP}

Increased intracranial pressure syndrome is a life-threatening neurological emergency. In the presence of $\mathrm{rICP}$, the pressure can be recorded invasively, besides non-invasive methods can also be used by advanced technology. These include pupillometric, dynamic, auditory, ophthalmic, and electrophysiological methods (206).

\subsubsection{Pupillometry}

Pupillometry is the objective assessment of pupillary light reaction using a non-invasive, automated device (Image 19). It is an easy-to-use device. However, ocular involvement, trauma, and patient incompatibility may limit the use of the device (206). Infrared light is used in pupillometric determination, as visible wavelength light can affect the light reaction. Many parameters related to the pupil's constriction-dilation functions can be measured in the pupillometric examination. These include maximum constriction amplitude (Mca), pupillary light reflex amplitude (PLRA), constriction velocity (CV), dilation velocity (DV), and neurologic pupillary index (NPi) . Mca is 


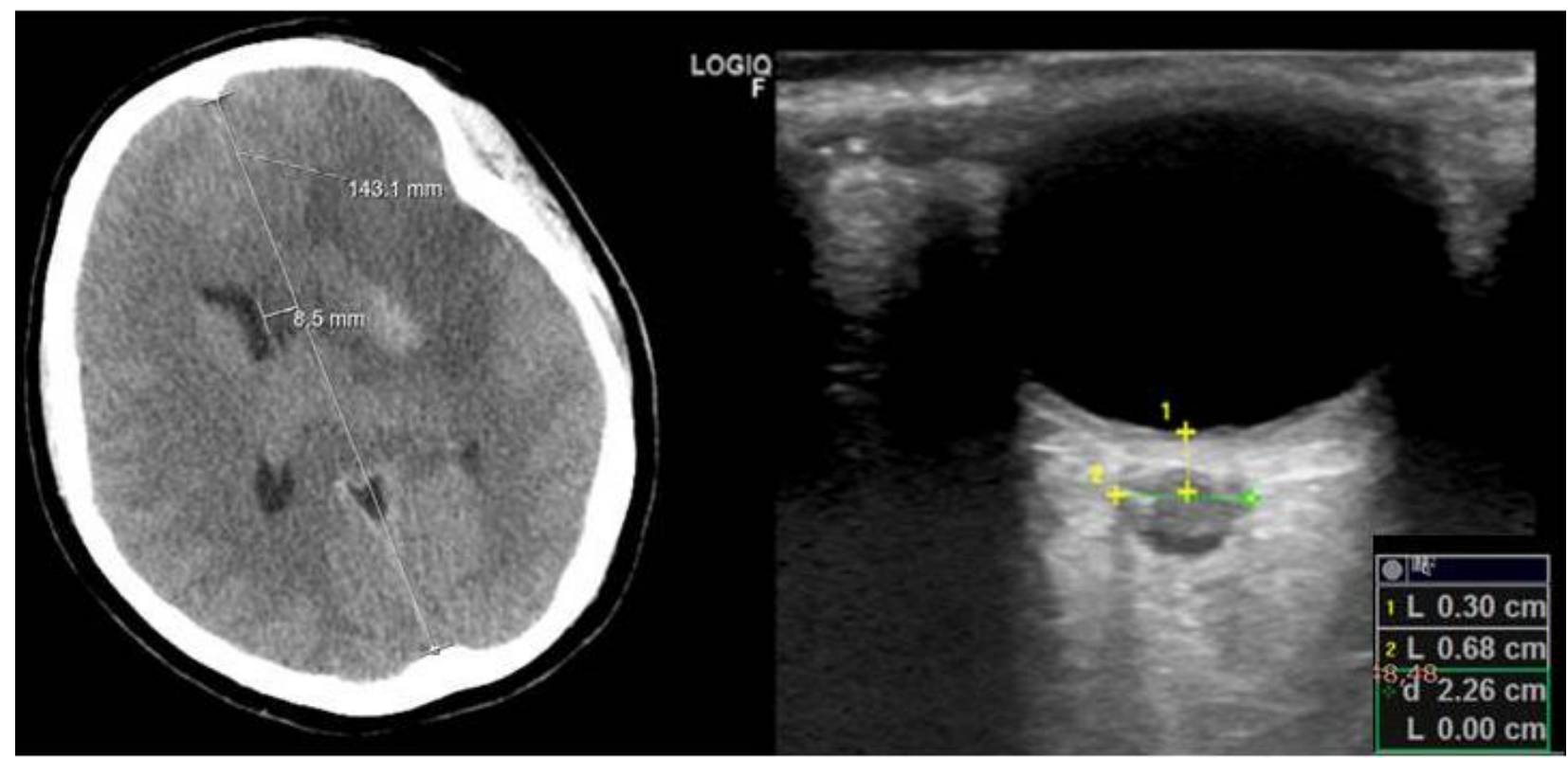

Image 18. In the neurological intensive care unit follow-up of the patient with left middle cerebral artery occlusion, 24-hour brain CT images show a wide infarct area causing effacement in the left frontotemporal cortical sulci and an $8.5 \mathrm{~mm}$ shift to the left of the midline (a). In the longitudinal section of the optic nerve, the optic nerve sheath diameter was measured $3 \mathrm{~mm}$ behind the eyeball with Orbital Bmode ultrasonography as $6.8 \mathrm{~mm}(\mathrm{~b})$.

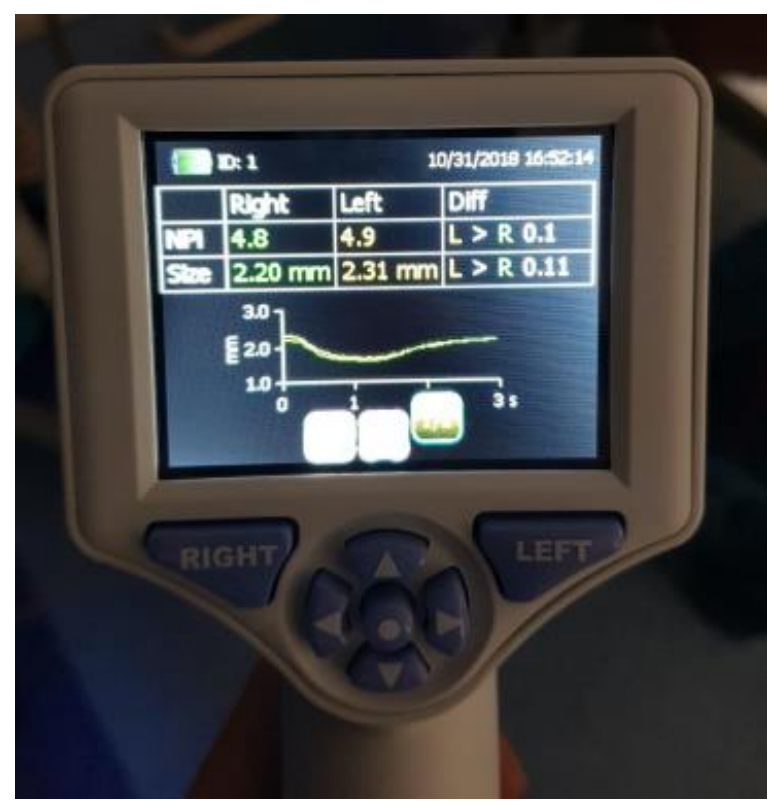

Image 19: Evaluation of a patient with normal intracranial pressure with an automated pupillometry device (OPD scan Macro Nidek()).

the difference between the baseline and minimum pupil diameter. CV and DV are obtained by dividing the MCA by the constriction and dilation times, respectively; PLRA is obtained by the percentage change of the pupil diameter compared to the baseline; NPi is calculated by evaluating the total latency, CV, and DV values with an algorithm. In $\mathrm{NPi}$, the overall score ranges from 0 to $5 ; 0$ reflects non-reactive pupils; $>3$ reflects normal values (207). Studies performed with invasive intracranial pressure monitoring reported that a decrease in CV below $0.6 \mathrm{~mm} / \mathrm{sec}$ is associated with an ICP value of $>20 \mathrm{~mm}-\mathrm{Hg}$ (208), and determined the mean ICP value as $19.6 \mathrm{~mm}-\mathrm{Hg}$ in patients with normal NPi, $30.5 \mathrm{~mm}-\mathrm{Hg}$ in patients with abnormal NPi and $33.8 \mathrm{~mm}-\mathrm{Hg}$ in patients with non-reactive NPi (209).

\subsubsection{Dynamic Methods}

Near-infrared Spectroscopy (NIRS): NIRS is a non-invasive method that makes measurements through two different frontal scalp sensors, the proximal sensor from the skin and bone in the relevant area and the distal sensor from $1 \mathrm{~cm}^{3}$ cerebral tissue under the bone. Cerebral oxygen saturation $\left(\mathrm{SO}_{2}\right)$ is assessed with the correlation coefficient (CC) between the absorption spectrum of total hemoglobin $(\mathrm{Hb})$ and oxygenated hemoglobin $\left(\mathrm{HbO}_{2}\right)$ in the measurement area $(210$, 211). It should be noted that misleading results can be obtained in the presence of advanced age, cerebral atrophy, or frontal trauma, as this method can measure up to a maximum depth of $2.5 \mathrm{~cm}$ 
(211). A recent study showed that $\mathrm{Hb} / \mathrm{HBO}_{2} \mathrm{CC}$ fluctuated and became positive in patients whose ICP was increased with an intraventricular infusion of Hartmann's solution, suggesting that it can detect vasogenic type subacute increases in ICP (212).

\subsubsection{Auditory Methods}

Tympanic membrane displacement occurs when perilymphatic pressure changes triggered by acoustic reflex cause oscillatory displacement in the tympanic membrane. Tympanic membrane displacement enables a non-invasive assessment of ICP (206). Despite some studies reporting sensitivity and specificity of ICP determination with tympanic membrane displacement as $94 \%$ and $100 \%$, respectively (213), it is not considered a suitable method for ICP assessment in most studies (214) due to measurement difficulties arising from compliance problems, intactness of the tympanic membrane and cochlear canal (214).

\subsubsection{Ophthalmic Methods}

Spontaneous venous pulsation (SVP) develops due to the gradient difference between the intraocular pressure of the retinal vein and the pressure of the cerebrospinal fluid (206). Theoretically, it is considered that SVP will disappear as a result of ICP elevation, and the presence of SVP is $94 \%$ sensitive for normal ICP (Wong and White 2013); conversely, the absence of SVP is not specific for raised ICP (206).

Evaluation of retinal nerve fiber layer (RNFL) thickness by optical coherence tomography; is another non-invasive method that may benefit the measurement of intracranial pressure (215), with the evaluation of peripapillary retinal pigment epithelium and Burch's upstream angles. However, its predictive power for intracranial pressure is not clear. (216). In addition, the slower development of optic disc edema compared to the ICP increase and the fact that the reliability of RNFL can change in conditions such as optic nerve atrophy are limiting factors (217).

\subsubsection{Electrophysiological Methods}

Even though studies on the indirect estimation of ICP with flash-visual evoked potential (F-VEP) latency and electroencephalogram (EEG) determined a correlation between prolongation of N2 latency in F-VEP and high ICP measured invasively (218); it should be noted that the reliability of this method will decrease in heavy optical refraction defects, pituitary masses, and metabolic disorders (206). On the other hand, a significant negative correlation was found between the ICP index obtained by EEG power spectrum analysis and the high ICP measured invasively $(\mathrm{r}=-0.84, \mathrm{p}<0.01)$. Further studies are needed for both assessments.

\subsection{Invasive Monitoring of Intracranial Pressure}

Increased intracranial pressure can cause decreased cerebral perfusion and is indicative of a poor prognosis. The aim of intracranial pressure measurement after stroke is to diagnose intracranial pressure increase early, to manage optimal cerebral perfusion pressure with early treatment, and hence to reduce mortality by preventing secondary brain damage and possible brain herniation. Invasive intracranial pressure monitoring is considered the gold standard for intracranial pressure measurement (219).

Invasive intracranial pressure monitoring; is generally indicated in patients with a Glasgow Coma Score (GCS) of 8 or less and at high risk of increased intracranial pressure (220, 221). Invasive intracranial pressure monitoring can also be considered in patients with a GCS greater than 8 , if the neurological examination cannot be followed up due to the need for long-term sedation and if a treatment that can increase intracranial pressure, such as positive end-expiratory pressure, is needed (220).

Its application is contraindicated in conditions such as coagulopathy, bleeding diathesis, severe infection, and hemodynamic instability, and since it is an invasive procedure, it may cause infection, bleeding, and technical complications $(220,222)$.

Depending on the technique, invasive intracranial pressure monitoring methods are classified according to the catheter and transducer location; measurements can be made from intraventricular, intraparenchymal, subarachnoid, subdural, and epidural areas. Intraventricular catheterization and intraparenchymal monitors are the most commonly used methods $(219,220$, $223,224)$.

The intracranial pressure threshold value was determined as $22 \mathrm{mmHg}$, especially in the IIPM literatüre obtained from patients with head 
trauma, and treatment was recommended for the values above (221). Values above this level are reported to be associated with increased mortality (223).

Although intracranial pressure monitoring provides clinical benefit, there is no consensus in the literature as to whether intracranial pressure monitoring provides a clinical advantage compared to management based solely on the patient's neurological examination and imaging findings. While some studies following invasive intracranial pressure monitoring have shown that intracranial pressure monitoring is associated with improved survival (225-228), there are also studies suggesting that intracranial pressure monitoring is not beneficial (229-231).

The clinical benefit of invasive monitoring of intracranial pressure in ischemic stroke is unclear. There is no recommendation for intracranial pressure monitoring in the guideline published by the American Heart Association/American Stroke Association in 2019 (232); the recommendations of the European Stroke Organization belong to the guideline published in 2008 (233). It is recommended to use invasive intracranial pressure monitoring in selected patient groups and keep cerebral perfusion pressure above 70 mmHg (233). Especially in the evaluations made in malignant cerebral infarcts, the $22 \mathrm{mmHg}$ intracranial pressure threshold value determined for post-traumatic brain injury remains a controversial issue, therefore the variability of these values for elderly and young patients should be taken into consideration, and further studies should be conducted (234).

Invasive intracranial pressure monitoring is rarely required in cases of cerebral venous thrombosis, and follow-ups in published case series have been made with a threshold value of $20 \mathrm{mmHg}$ intracranial pressure, and lack sufficient data to make a definite recommendation (235, 236).

It is known that increased intracranial pressure is also a negative prognostic factor in patients with spontaneous intracerebral hemorrhage. The available studies recommend the use of IIPMs in this patient population with a Class IIb level of evidence. Patients with a GCS score of $\leq 8$, transtentorial herniation, or evidence of significant intraventricular hemorrhage or hydrocephalus are candidates for invasive intracranial pressure monitoring. Maintaining cerebral perfusion pressure at 50 to $70 \mathrm{mmHg}$ ' is recommended. The $22 \mathrm{mmHg}$ intracranial pressure threshold value determined for this population after traumatic brain injury remains a controversial issue, and further studies are required (237-239).

In conclusion, the guidelines are mainly based on the principles for the use of intracranial pressure monitoring in traumatic brain injury. Indications for the use of intracranial pressure monitoring in stroke, threshold values for intracranial pressure, and cerebral perfusion pressure are not yet clearly defined.

\section{Treatment of Cerebral Edema in Stroke 4.1 General Principles}

During the treatment of cerebral edema that develops after acute stroke, the aim should be to transport blood containing ideal levels of oxygen, carbon dioxide, and glucose, with normal $\mathrm{pH}$ and electrolyte levels to the stressed cerebral tissue prone to injury and to keep the brain temperature at a normal level (1). These patients should be followed up in Neurology Intensive Care Units. Normocarbia should be provided, and intubation and mechanical ventilation should be evaluated in patients with impaired consciousness. The head should be kept elevated at least 30 degrees, and it should be ensured that there is no wrapping or compression on the neck that would impair venous return. It should be kept in mind that an increase in intra-abdominal or intrathoracic pressure can increase intracranial pressure and decrease cerebral perfusion, therefore medical conditions that will increase intra-abdominal and intrathoracic pressure should be prevented or treated. It is recommended to insert a central venous catheter and an arterial route from the jugular or subclavian vein in patients who are intubated and need mechanical ventilation. The patient should be kept euvolemic. Fluid resuscitation should be performed with isoosmolar solutions, hypotonic and hypoosmolar solutions should be avoided. It should be noted that very high blood pressure values may lead to hemorrhagic transformation or enlargement of the hematoma in the infarct area For blood pressure regulation, intravenous nicardipine or intravenous esmolol infusion, which can be manipulated more easily according to the patient's condition and response, is preferred rather than oral/enteral antihypertensives (219). In hyperglycemic, 
patients, blood sugar should be kept between 140$180 \mathrm{mg} / \mathrm{dl}$. Normothermia should be targeted. If there is a fever, the body temperature can be lowered with superficial cooling and $500 \mathrm{mg}$ intravenous paracetamol infusion every 6 hours after the necessary samples are taken to find the focus of infection $(11,240)$. If there is no seizure, antiepileptic prophylaxis is not recommended; if there is no infection and fever, antibiotic prophylaxis is not recommended. Deep vein thrombosis prophylaxis with low-molecularweight heparin should be initiated early. After stabilizing cerebral and systemic hemodynamics, enteral nutrition should be started in the early period, albeit in very small doses (trophic nutrition infusion at a rate of $10-20 \mathrm{ml} /$ hour) (241).

The pushing pressure created by solute particles in a liquid is called osmotic pressure. If the amount of solute in the fluid compartments divided by a semi-permeable membrane is different, the osmotic pressure on the two sides is also different. The osmotic gradient between the two sides of this membrane, which is permeable to water but not permeable to particles, is tried to be equalized by the passage of water to the side where the pressure, that is, the amount of particles, is high. This is called the osmotic balance principle. The mechanism of action of osmotic drugs such as mannitol and hypertonic saline used in the treatment of cerebral edema is to accumulate on the blood side of the blood-brain barrier and increase the osmotic pressure, thus allowing excess water on the brain side of the blood-brain barrier, that is, cerebral edema, to pass into the intravascular space by passive diffusion. For this to happen, the blood-brain barrier must be intact $(36,242)$. The rationale of decompressive hemicraniectomy surgery is to overcome the Monro-Kellie principle. The rapid decrease in intracranial pressure will indirectly increase cerebral blood flow (243).

Conservative and specific treatments to be used in the treatment of brain edema are given below with an algorithm (Image 8).

\section{Edema}

4.2. Treatment of Cytotoxic Cerebral

Cytotoxic cerebral edema treatment in ischemic stroke cases can be examined in two groups, as medical and surgical. Medical treatments are considered hyperosmolar treatments; surgical treatments decompressive craniectomy (244).

Hyperosmolar agents used in the treatment of cytotoxic edema in neurointensive care clinical practice are mannitol and hypertonic saline. Urea has been used for a while but is no longer used due to its side effects.

In the last decade, hypertonic saline has been preferred more in the treatment of cerebral edema compared to mannitol. It is primarily preferred because of its effect that starts in as little as five minutes and lasts longer than 12 hours, and its ability to provide and maintain intravascular volume. However, there are no randomized controlled trials showing the superiority of hypertonic saline to mannitol. Therefore, the decision of which of these two options to choose should be specific to the patient. Mannitol should be preferred in patients with a known or clinically determined history of pulmonary edema or congestive heart failure, while hypertonic saline should be preferred in patients with hypotension and hypovolemia risk since it does not have a diuretic effect. In the latest guidelines, prophylactic mannitol administration is not recommended in ischemic stroke cases, and in cases with inadequate response to mannitol treatment, hypertonic saline administration is recommended (245).

\subsection{Treatment of Ionic Edema}

Cerebral edema after ischemic stroke includes cytotoxic edema, ionic edema, and vasogenic edema (174). Ionic edema developing after cerebral ischemia is thought to follow cytotoxic edema. It occurs when the blood-brain barrier is still intact, especially as a result of the dysfunction of the ion and water channels on the endothelial side of the barrier, and the consequent transport of free water without macromolecules into the cerebral extracellular space in the lumen of the vessel, along with the ionic transition (40). Ionic edema is a subtype of extracellular edema lacking albumin. It has been shown to develop independently of cytotoxic edema. Treatments for ionic edema formation, especially on the endothelial side of the BBB, target Sur1-Trpm4, $\mathrm{Na}^{+}-\mathrm{K}^{+}$-cotransporter-1 (NKCC1), aquaporin-1 and aquaporin-4 channels, and $\mathrm{Na}^{+}-\mathrm{H}^{+}$channel. The aim is to prevent or reduce ionic edema development in the presence of cerebral ischemia by using pharmacological agents that block these 
TREATMENT OF CEREBRAL EDEMA IN STROKE

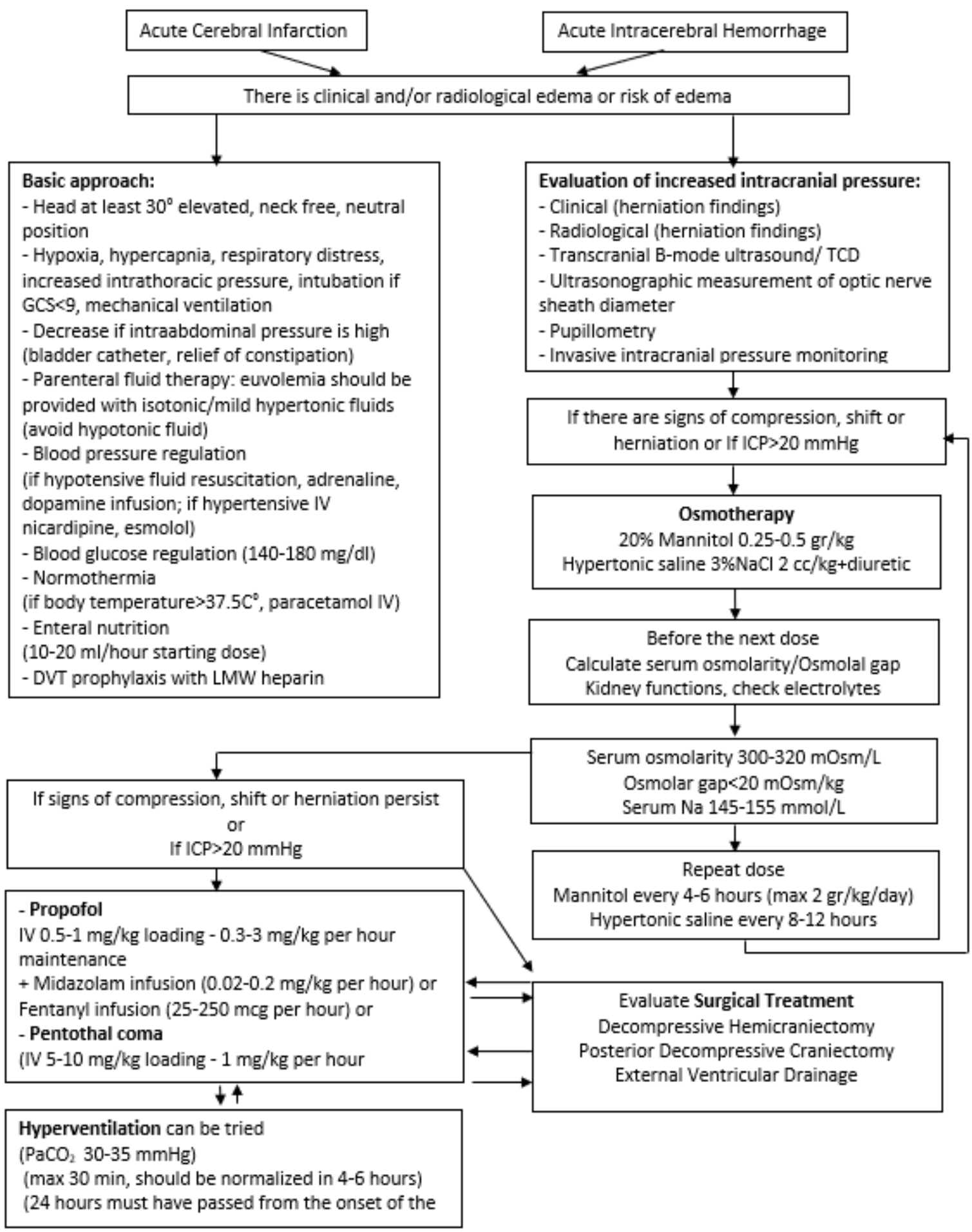

Figure 8. Cerebral edema treatment algorithm in stroke. 
ion channels and transporters (246-249). The Sur1-Trpm4 channel is involved in both cytotoxic and ionic edema and is the most promising therapeutic target for edema therapy. Sur1-Trpm4 channel inhibitors are the only drug group entering clinical trials for the treatment of cerebral edema in ischemic stroke.

\subsection{Treatment of Vasogenic Edema}

Vasogenic edema occurs with disruption of the cerebral capillary endothelium in the bloodbrain barrier (BBB). With the increase in capillary permeability, osmotic and hydrostatic forces act together, plasma filtrate containing plasma proteins passes freely into the extracellular fluid, and the edema reaches its maximum level approximately 24-48 hours after the capillary endothelial disruption. After disruption of the blood-brain barrier, connections are formed between the brain interstitial space and vessels. Plasma proteins and other macromolecules pass into the extracellular space of the brain (44). Blood-brain barrier disruption and vasogenic edema are commonly observed in cerebral trauma, hemorrhage, and the second phase of ischemia (3). Vasogenic edema increases the functional loss of the patient and the risk of mortality in both intracerebral hemorrhage (ICH) and cerebral infarction in stroke patients.

Vasogenic edema can be considered as an ultrafiltrate of blood. Vascular endothelial growth factor (VEGF) and free radical matrix metalloproteinases (MMP) are involved in the development of vasogenic edema. Mast cells are also involved in this process (250). Aquaporin-4 (AQP4) helps to reduce vasogenic edema. Vascular endothelial growth factor, BBB, Na-Ca exchanger, AQP4, and mast cells have been identified as potential targets in the treatment of vasogenic edema. Aquaporin-4 has a significant role in the development of cytotoxic edema as well as in the clearance of excess parenchymal fluid caused by vasogenic edema. Various aquaporin-4-linked compounds are under development (249).

The edema that occurs in intracerebral hemorrhage is generally called perihematomal edema (140, 251). Inflammation plays an important role in edema that develops after ICH. Since polymorphonuclear cell (PMN) infiltration and microglia activation are considered the main causes in the early period, treatments for their inhibition are being investigated. It is emphasized that, consequently, BBB permeability can be improved, and perihematomal axonal damage can be reduced. BBB thought to be impaired by thrombin can be inhibited by thrombin inhibitors, but there will be an adverse effect on hemostatic function. The effects of immunomodulatory drugs such as matrix metalloproteinase inhibitors, TNF alpha neutralizing antibodies, and fingolimod on cerebral edema are under investigation (252).

Keeping the airway open and preventing hypoxia at the stage before vasogenic edema develops, along with well-controlled blood pressure, are the basic measures in stroke patients. Standard pharmacological treatments can be summarized as osmotherapy (mannitol, hypertonic sodium), agents that reduce the production of cerebrospinal fluid (acetazolamide, furosemide), glucocorticoids (dexamethasone), and barbiturates. Other treatment methods are hyperventilation and hypothermia applications and surgical treatments such as decompressive craniectomy.

However, in the treatment of vasogenic edema due to ischemic stroke, the use of hypothermia and barbiturates is not recommended. The data on hypothermia in hemorrhagic stroke available so far are insufficient (251, 253).

Dexamethasone inhibits cyclooxygenase-2, which plays a role in the production of eicosanoids, and normalizes endothelial cell membrane permeability. It is effective in the treatment of vasogenic edema due to tumor, abscess, head trauma. However, it is not recommended due to the lack of evidence for its efficacy in the treatment of cerebral edema and increased intracranial pressure in ischemic stroke and intracerebral hemorrhage and its potential to increase infection complications $(245,254)$.

Short-term moderate hyperventilation $\mathrm{PCO}_{2}$ target $30-34 \mathrm{~mm} \mathrm{Hg}$ ) can be used to gain time. It can be used as a salvage treatment during preparation for surgery in patients with brain stem herniation findings, but it should be noted that hyperventilation may cause brain ischemia if hypocapnia is deep $(<30 \mathrm{~mm} \mathrm{Hg})$ and prolonged (254).

\subsection{Future Treatments for Cerebral Edema}

Cerebral edema is extremely dangerous. Severe cerebral edema after stroke can increase mortality rate up to $80 \%$ and is an important 
predictor of poor survival $(250,255)$. Therefore, an effective antiedema treatment in the early stage of stroke is important. Nevertheless, treatment options have been limited due to the incomplete understanding of the pathophysiology of cerebral edema, and it has been applied to reduce adverse outcomes such as herniation, not to prevent edema, regardless of the underlying pathological process. Recently, many drugs have been investigated to prevent the development of cerebral edema after stroke. This section discusses the potential mechanisms of action and efficacy of these drugs.

Currently, there are no drugs approved for clinical application among the drugs to be described below. The efficacy of these drugs should be approached with caution due to low drug delivery into the brain and the fact that cerebral edema occurs with more than one factor and mechanism (256).

1. SUR1-TRPM4 channel inhibitors: SUR1TRPM4 (Sulfonylurea receptor 1- transient receptor potential melastatin 4) channel is an important molecular structure in focal cerebral ischemia. Following ischemia, these channels are upregulated in all cells of the neurovascular unit. In the absence of ATP after brain damage, it allows $\mathrm{Na}^{+}$osmolytes to enter the cell, leading to cell edema and death (44).

Glibenclamide protects the tissue against ischemic damage by blocking the SUR1-TRPM4 channel induced by ischemia and may reduce cerebral edema.

Preclinical studies have shown that SUR1TRPM4 blockade with glibenclamide reduces cerebral edema, improves functional outcomes, and decreases mortality (109). Combined treatment with glibenclamide and therapeutic hypothermia in a middle cerebral artery occlusion rat model demonstrated a synergistic neuroprotective effect by reducing edema and improving neurobehavioral function (257). The retrospective analyzes of diabetic patients who used and did not use glibenclamide during acute ischemic stroke determined that functional recovery and survival were higher in those using glibenclamide (258, 259). In a phase 2 study, intravenous glibenclamide reduced cerebral edema without severe hypoglycemia and was well tolerated (260). Glibenclamide requires intravenous administration, which limits its application in out-of-hospital settings. However, oral preparations can also be given in the early stages of edema. Although glibenclamide can reduce cerebral edema, it can cause hypoglycemia.

Glimepiride is a second-generation sulfonylurea. Hypoglycemic events were observed less frequently than with glibenclamide. Glimepiride was as effective as glibenclamide in reducing stroke and cerebral edema in mouse models (261).

Resveratrol is a natural product found in various plants (RSV, trans-3, 4', 5-trihydroxystilbene), has protective properties in ischemia, can reduce cerebral edema, and prevent $\mathrm{BBB}$ damage (262). Moreover, resveratrol has been reported to reduce SUR1 and AQP4 expression and edema formation in animal models of transient ischemia (263).

2. Drugs related to vascular endothelial growth factor: Vascular endothelial growth factor (VEGF) is a mitogen that provides the formation of new blood vessels and increases vascular permeability. In animal models, VEGF has been shown to reduce infarct volume and cerebral edema when given before middle cerebral artery occlusion (264) and increase BBB permeability and cerebral edema when given after ischemia (265).

3. Aquaporin blockers: Aquaporins (AQP) contribute to cytotoxic edema; especially, AQP4 plays an important role in the water balance in the brain in stroke patients (266). AQP4 has a bidirectional effect on water transport; it plays a role in both the formation and prevention of edema. AQP4 can increase the formation of cytotoxic and ionic edema in the early period. As the edema passes into the vasogenic stage, AQP4 begins to contribute to the clearance of cerebral edema (267). It is unclear when to administer AQP4 inhibitors for the prevention or treatment of ischemic cerebral edema. It has been suggested that AQP1 may also play a role in the development of cerebral edema and that AQP1 inhibitors such as fullerenols may reduce edema (268).

\section{Ion channel blockers:}

NKCC1 inhibitors: $\mathrm{Na}^{+} \mathrm{K}^{+} \mathrm{Cl}^{-}$cotransporter protein-1 (NKCC1) and $\mathrm{Na}^{+} \mathrm{K}^{+}$cotransporter protein-2 (KCC2)are cationic $\mathrm{Cl}^{-}$cotransporters with opposing effects on intracranial water and electrolyte balance. They play an important role in the formation of both cytotoxic and ionic edema. Bumetanide, which inhibits NKCC1, can reduce cytotoxic edema (269). Bumetanide has been 
reported to reduce cerebral edema in an ischemiareperfusion model without altering KCC2 protein expression (270). Bumetanide can be effective in both acute and chronic phases of cerebral ischemia (271).

$\mathrm{Na}^{+}-\mathrm{H}^{+}$Exchanger inhibitors: The $\mathrm{Na}^{+} \mathrm{H}^{+}$plays an important role in the early stage of postischemia cerebral edema. Hypoxia may increase the formation of cerebral edema by stimulating the $\mathrm{Na}^{+} \mathrm{H}^{+}$exchanger activity in the BBB. Intravenous injection of a $\mathrm{Na}^{+} \mathrm{H}^{+}$exchanger inhibitor (HOE-642; compound RU-1355) has been shown to reduce cerebral edema by significantly reducing $\mathrm{Na}$ concentration (272).

$\mathrm{Na}^{+} \mathrm{Ca}^{++}$Exchanger inhibitors: Maintaining intracellular $\mathrm{Ca}^{++}$homeostasis is crucial for the protection of the BBB. $\mathrm{Na}^{+} \mathrm{Ca}^{++}$exchanger has been shown to play a role in vasogenic edema and $\mathrm{BBB}$ destruction. In addition, $\mathrm{Na}^{+} \mathrm{Ca}^{++}$exchanger inhibitors (SEA0400) can reduce cerebral edema and cerebral infarct volume after cerebral ischemia (273).

5. Conivaptan: Arginine vasopressin and its receptors $\mathrm{V} 1 \mathrm{a}$ and $\mathrm{V} 2$ play an important role in cerebral edema after ischemic stroke (274). Conivaptan, a V1a and V2 receptor blocker, can reduce cerebral edema and blood-brain barrier damage after ischemia (275). It has been shown that conivaptan treatment started 3 hours after ischemia can reduce edema and improve neurological deficit (276).

6. Fingolimod: Fingolimod is a Sphingosine1-phosphate (S1P) receptor modulator approved in 2010 for the treatment of multiple sclerosis (277). Preclinical trials have shown that fingolimod reduces cerebral edema in models of intracranial hemorrhage and ischemic stroke (278, 279).

7. MicroRNA: Expression of microRNA-1 (Mir-1) is related to ischemic injury and cellular apoptosis. PIn preclinical models, treatment with anti-miR-1 reduced infarct volume, while treatment with miRNA-1 antagomir significantly reduced brain edema, BBB permeability, infarct volume, and neurologic injury (280).

8. BBB protective agents: Vasogenic edema occurs after BBB injury. Therefore, maintaining BBB integrity is an important outcome measure for antiedema treatments. Alpha-tocopherol, 8methoxypsoralen, 3-aminobenzamide, and calcitriol show antiedema activity by protecting the BBB (261).
Potential drugs targeting BBB include magnesium sulfate, ulinastatin, ginkgo diterpene lactone meglumine injection, olaparib, recombinant human erythropoietin, and 1trifluoromethoxyphenyl-3-(1-propionylpiperidin4-yl) urea $(250,281)$.

\subsection{Osmotherapy}

Osmotherapy is the use of osmotically active substances to reduce the volume of intracranial contents. The primary purpose of osmotherapy is to reduce the intracranial volume by creating an osmotic difference between blood and brain and thereby removing the free water accumulated due to cerebral edema from the intracellular and interstitial spaces of the brain. Osmotherapy shifts excess water from the intracellular and interstitial spaces to the intravascular space, reducing increased intracranial pressure and increasing serum osmolarity (219). Normal serum osmolality ranges from 280-290 mOsm/kg and should be maintained in the range of $300-320 \mathrm{mOsm} / \mathrm{kg}$ during treatment. Treatment is carried out by intravenous administration of osmotic agents. In patients with traumatic brain injury, intracranial pressure has been shown to decrease by $14 \mathrm{mmHg}$ 1 hour after administration of mannitol and by 10 mmHg 1 hour after hypertonic saline (1). Osmotherapy is used in primary medical therapy to minimize midline shift and reduce the intracranial volume in large hemispheric strokes. In the AHA/ASA (American Heart Association/American Stroke Association) 2018 guideline, hyperosmolar therapy is at Class IIb, Level of Evidence C-LD, and its use as prophylactic treatment is not recommended (244). Which agent to prefer is decided by considering the patient's blood pressure, cardiac output, and renal functions. While making this decision, it should be kept in mind that mannitol is a dehydrating drug and hypertonic saline is a drug that increases the intravascular volume (1). Mannitol and hypertonic saline can also be used alternately, considering their side effects.

After an ischemic stroke, cerebral edema begins to develop in 1-3 days and reaches its most severe level on days 3-5. It gradually decreases after day 7 and disappears in the 3rd week. The use of osmotic agents for longer than 3-5 days is not recommended due to complications and tolerance development to their effects. For this reason, it would be appropriate to use 
osmotherapy as a weapon in the phase where edema develops most, not in the hyperacute period. When to start osmotic agents should be decided according to the patient's clinical condition, neuroimaging findings, and other organ functions. It is not recommended to routinely start aggressive osmotherapy at first in every stroke patient at risk of developing cerebral edema. On the other hand, malignant cerebral edema is a catastrophic condition that develops rapidly in the first 24 hours and leads to herniation. If there are clinical and radiological signs of malignant brain edema, osmotherapy should be started earlier. In intraparenchymal hemorrhages, if the developed perihematomal edema requires treatment, osmotherapy can be started in the first 24 hours $(1,244,245,(282)$.

\subsubsection{Mannitol}

It is the main osmotic agent used in the clinic, owing to its half-life of 2-6 hours, ease of preparation, and chemical stability. Mannitol is available commercially as $20 \%$ ready-made solutions of 100, 150, 250, and $500 \mathrm{ml}$. Mannitol $\left(\mathrm{C}_{6} \mathrm{H}_{8}(\mathrm{OH})_{6}\right)$ is a sugar alcohol with a weight of 182.17 daltons and an osmolarity of 1100 $\mathrm{mOsm} / \mathrm{L}$. It is excreted in the urine without being metabolized in the circulation. The recommended dose for the treatment of cerebral edema is 0.5-1.5 $\mathrm{g} / \mathrm{kg}$. It should not exceed $2 \mathrm{gr} / \mathrm{kg}$. Each dose is rapidly infused intravenously over 20-30 minutes. With bolus administration, its intracranial pressure-lowering effect starts in 5 minutes, reaches its peak in 20-60 minutes, and continues for 1.5-6 hours. Rapid infusion can be repeated every 4-6 hours at a dose of $0.25-0.5 \mathrm{~g} / \mathrm{kg}$, depending on the severity of ICP (244). Serum osmolality can be tolerated up to $360 \mathrm{mOsm} / \mathrm{kg}$. During treatment, the osmolal gap should not exceed $55 \mathrm{mOsm} / \mathrm{kg}$. Increasing the osmolal gap above $60-75 \mathrm{mOsm} / \mathrm{kg}$ or administering a total of $1100 \mathrm{~g}$ of mannitol increases the risk of acute renal failure. In addition, if the serum osmolarity exceeds $320 \mathrm{mOsm} / \mathrm{L}$, mannitol administration should be discontinued to prevent the development of acute renal failure and metabolic acidosis (244). Since it cannot be reabsorbed from the proximal tubules of the kidneys, it also reduces the reabsorption of water with its osmotic effect and acts as a diuretic. Osmotic diuretics reduce the renal reabsorption of $\mathrm{Na}, \mathrm{K}, \mathrm{Cl}, \mathrm{P}, \mathrm{Ca}, \mathrm{M}$, urea, and uric acid along with water. It is crucial to maintain the hyperosmotic euvolemic state during treatment; otherwise, dehydration, hypovolemia, hypotension, increased intracranial pressure, decreased cerebral perfusion pressure, acute renal failure, and electrolyte imbalance may develop. After 1-3 days of repeated dosing, the dose should be gradually tapered off to prevent rebound effect (240). Conditions in which mannitol should not be used are given in Table 3.

Table 3. Mannitol contraindications.

\begin{tabular}{l} 
Conditions for which Mannitol is Contraindicated \\
\hline Decreased renal perfusion \\
Acute renal failure \\
Shock \\
Sepsis \\
Serum osmolarity $>320 \mathrm{mOsm}$ without osmotherapy \\
Nephrotoxic drug use
\end{tabular}

\subsubsection{Hypertonic Saline}

It is a saline solution prepared by dissolving $\mathrm{NaCl}$ in water. Hypertonic saline solution (HTS) has been the choice of neuro-intensive care over the last few years. The concentration of hypertonic saline solution used can vary and may be $3 \%, 5 \%$, $7.5 \%, 10 \%$, or $24.3 \%(1.7-30 \%)$ and the dose is 2 $\mathrm{g} / \mathrm{kg}$. In clinical practice, it is administered by intermittent slow infusion (3\% saline solution at a dose of $2 \mathrm{cc} / \mathrm{kg}$ in 20-30 minutes or $15-30 \mathrm{ml}$ in a concentration of $23.4 \%$ in 10 minutes) or by continuous infusion at a $3 \%$ concentration of 1-1.5 $\mathrm{ml} / \mathrm{kg} /$ hour. The duration of action is 2-6 hours. The infusion can be repeated every 8 hours. The osmolarity of $3 \% \mathrm{NaCl}$ is $1027 \mathrm{mOsm} / \mathrm{L}$; it can be administered through a peripheral vein. The osmolarity of $7.5 \% \mathrm{NaCl}$ is $2545 \mathrm{mOsm} / \mathrm{L}, 23.4 \%$ $\mathrm{NaCl}$ is $8004 \mathrm{mOsm} / \mathrm{L}$, and must be administered via the central venous route. In order to keep the serum sodium concentration between 145-155 $\mathrm{mmol} / \mathrm{L}$, it is essential to monitor every 4-6 hours. Hypertonic saline has no diuretic effect, hence post-infusion diuretics may be required (244).

In the case of resistant cerebral edema, mannitol and hypertonic saline can be administered alternately. According to the " $2 / 2 / 2$ " rule, mannitol is administered first at the 0th hour, and hypertonic saline is administered at the 2nd hour. At the 4th hour, the patient's laboratory parameters are checked. If there is no significant abnormality in laboratory parameters based on the evaluation, a new 6-hour cycle is started (283).

Hypertonic saline normalizes the resting membrane potential and has an anti-inflammatory 
effect. It increases serum sodium level, brain $\mathrm{pH}$, and brain tissue oxygenation (240, 245). According to the latest guidelines from the "Neurocritical Care Society", bolus administration of hypertonic saline solution is recommended for the treatment of acute ischemic stroke, subarachnoidal hemorrhage, and intracerebral hemorrhage to reduce intracranial pressure and cerebral edema (evidence-quality is very low). However, there are insufficient data on the improvement of clinical neurological outcome (245). Possible side effects are rebound cerebral edema, hyperchloremic metabolic acidosis, phlebitis, congestive heart failure, transient hypotension, hemolysis, hypokalemia, renal failure, osmotic demyelination, subdural hemorrhage, epilepsy, and muscle twitching (240).

Studies have shown that HTS is more effective than mannitol in controlling intracranial pressure, but there is no difference in clinical outcome $(242,284)$. Compared to Mannitol; evidence suggests that HTS administration causes a shorter cumulative period of intracranial hypertension and increased cerebral perfusion pressure and has a longer-lasting improvement effect on cerebral blood flow and cerebral perfusion pressure. A meta-analysis of 12 randomized clinical trials comparing both osmotic agents found no difference in neurological function and mortality $(219,285)$. The use of continuous infusion to maintain the hyperosmolar state remains controversial (242). Combining osmotherapy with other treatment mechanisms has the potential to more effectively treat cerebral edema. Hyperosmolar agent selection, timing, and dosing regimen should be tailored to the patient at the optimum level (286). Existing comorbidity, volume status, and serum osmolarity can be decisive in the selection of the agent, and the aim should be to keep the possible side effects at a minimum (219) (Table 4). Based on the available evidence, the clinician should decide on the most appropriate treatment for the individual by balancing efficacy and safety (245).

Table 4: Side effects of hypertonic saline and mannitol.

Mannitol
- Hyponatremia, Hypochloremia, Hypokalemia, Alkalosis
After 200cc bolus
serum Na drops by $1.7 \mathrm{mEq} / \mathrm{L}$
serum $\mathrm{Cl}$ drops by $1.7 \mathrm{mEq} / \mathrm{L}$
- Acute renal failure / Acute tubular necrosis (over $200 \mathrm{gr} /$ day)
- Hypotension
- Congestive heart failure and pulmonary edema

Hypertonic Saline

- Hypernatremia, Hyperchloremia, Hypokalemia, Acidosis

- Hypotension during infusion

- Hypervolemia

- Pulmonary edema

- Acute renal failure

- Hemolysis

- Osmotic demyelination syndrome

- Arrhythmia

- Coagulopathy

- Phlebitis

- Seizure

- Muscle fasciculation

\section{Monitoring \\ 5.2 Osmometry in Osmotherapy}

Close follow-up of hyperosmolar therapy, which is a frequently used approach in the treatment of $\mathrm{rICP}$, is recommended to minimize kidney-related side effects. In order to achieve these goals, first of all, it is necessary to know what the concepts of osmolality and osmolarity mean. While osmolality expresses the number of moles of solutes per kilogram of a solution, osmolarity expresses the same number of moles per unit volume of solution. In short, the unit of osmolality is $0 s m / \mathrm{kg}$, the unit of osmolarity is Osm/L (286).

Under physiological conditions, the serum osmolality level is $275-295 \mathrm{mOsm} / \mathrm{kg}$. Although direct measurement of osmolality is possible, determining osmolality using certain formulas is a more practical approach in a person who has not received any hyperosmolar therapy. In fact, as can be seen below, since solute amounts per volume are used in the formulas, it is osmolarity that is calculated, not osmolality. However, since osmolality and osmolarity are very close to each other in dilute solutions such as body fluids, these terms are used interchangeably in clinical practice (287). Here are a few of the various formulas that can be used to calculate osmolarity: 


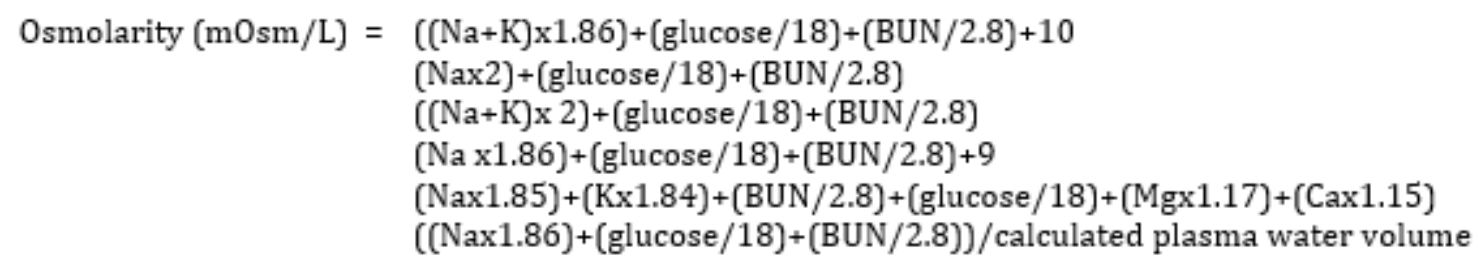

\section{In the formulas, electrolytes are in $\mathrm{mEq} / \mathrm{L} ;$ glucose and BUN in $\mathrm{mg} / \mathrm{dl}$.}

The purpose of using hyperosmolar agents is to increase this osmolality/osmolarity level and to ensure the passage of free water from the intercellular and intracellular space into the vessel, thanks to the osmotic pressure formed. Since it is not possible to estimate the amount of the increase with formulas, the blood osmolality level of patients receiving hyperosmolar therapy should be measured directly in the laboratory using osmometers (287).

The difference between the measured osmolality and the calculated osmolarity from the formulas is called the osmolal gap; this gap gives information about the solute load that is not reflected in the formulas and can only be detected by direct measurement, which is of great importance in cases such as ethanol or methanol poisoning, or in patients receiving hyperosmolar therapy such as mannitol. The gap is below 10 mOsm/kg under normal conditions. Although there is no significant difference between the formulas above in the determination of the calculated osmolarity amount to be subtracted from the measured osmolality amount, the most accurate osmolal gap determination has been shown with the formula in the first line (287).

In patients undergoing hyperosmolar therapy with mannitol, the determination of osmolality should be performed at baseline blood mannitol concentration, i.e., before the upcoming next dose of mannitol (usually 1 hour before). This measurement should be done at least once a day. Despite the classical recommendation that the measured osmolality levels should not exceed 320 $\mathrm{mOsm} / \mathrm{kg}$ to prevent the side effects of treatment, especially of acute renal failure, recent literature indicates that this threshold value can be exceeded, and this value is not reliable enough to predict kidney-related complications. Another goal of treatment is to have an osmolal gap below 20 $\mathrm{mOsm} / \mathrm{kg}$; a high osmolal gap level is an indication that mannitol is still present in the blood. In such a condition, it is recommended to skip the next dose of mannitol and modify the general dosing schedule (dose reduction or increasing the dose intervals) $(245,286)$.

In the case of hyperosmolar therapy with hypertonic sodium, direct measurement of osmolality is not required since the hypernatremia resulting from this treatment leads to an osmolarity change that can be calculated by the formulas above. However, instead of osmolarity calculation, the practical recommendation is to target a plasma sodium concentration of 145-155 $\mathrm{mEq} / \mathrm{L}$ with hypertonic sodium therapy and not to exceed $160 \mathrm{mEq} / \mathrm{L}$ to minimize side effects (245, 286).

In conclusion, the main goal in the follow-up of hyperosmolar therapy is to prevent acute renal failure, the most common side effect of treatment. The literature has shown that the ideal method to reduce this risk is the direct determination of serum mannitol level. However, since this is not a practical and widely applicable method, risk management can be done by methods such as osmolality determination, osmolality gap calculation, and sodium level monitoring (245, $287,288)$.

\subsection{The Role of Corticosteroids in the Treatment of Cerebral Edema}

Cytotoxic brain edema is observed in conditions such as ischemia, metabolic diseases, and trauma, while vasogenic brain edema is observed in tumors, infection, inflammation, and hyperosmolar conditions. Since the physiopathology of cytotoxic and vasogenic brain edema is different, their responses to corticosteroids are also different. Corticosteroids are immunosuppressive agents; they may be effective in alleviating the effects of vasogenic cerebral edema, especially due to space-occupying lesions, reducing intracranial pressure, and strengthening the blood-brain barrier. It should be used by considering the benefit-harm balance due to side effects such as infections secondary to 
immunosuppression, impaired blood sugar regulation, increased risk of gastrointestinal bleeding, and delayed wound healing) (289).

\subsubsection{Ischemic Stroke}

Cytotoxic edema occurs primarily in ischemic stroke, but vasogenic edema can also develop in case of disruption of the blood-brain barrier. Although corticosteroids are theoretically expected to be beneficial for vasogenic edema after ischemic stroke, clinical studies do not support this expectation. Studies on the use of corticosteroids in ischemic stroke are mostly out of date. The consensus regarding the use of corticosteroids in stroke is in the suspected/definite diagnosis of vasculitis. A Cochrane meta-analysis on eight studies involving 466 patients that received corticosteroids in the first 48 hours reported no benefit in 1-year survival or neurological recovery (290). In the AHA/ASA 2019 guideline, the use of corticosteroids is not recommended in cerebral edema after an ischemic stroke due to the lack of evidence of its benefit and its potential to increase the risk of infectious complications and to be harmful (232).

\subsubsection{Intracerebral Hemorrhage}

The European Stroke Organization (ESO) guideline does not recommend the use of corticosteroids in spontaneous intracerebral hemorrhage (291). In another recent guideline, corticosteroid use is also not recommended to improve neurologic prognosis in intracranial hemorrhage [245]. Despite a few publications reporting the positive effect of intravenous dexamethasone on functional recovery and mortality (292), most studies do not recommend its use because it increases infectious complications, increases mortality, and does not have a positive effect on neurological recovery. In primary intracerebral hemorrhage and aneurysmatic subarachnoid hemorrhage, there was no difference in neurological improvement between steroid use and standard care. A Cochrane review found no differences in subarachnoid hemorrhage treated with hydrocortisone compared to placebo (293). Natriuresis-induced hyponatremia is common after aneurysmatic subarachnoid hemorrhage; it induces osmotic diuresis, decreases blood volume, and thereby increases the risk of cerebral vasospasm. In a randomized controlled trial, patients with subarachnoid hemorrhage receiving hydrocortisone had better control of sodium, fluid balance, and plasma osmolarity; however, there was no difference in symptomatic cerebral vasospasm and clinical outcomes (294). In the light of this information, there is no evidence to recommend the use of steroids in subarachnoid hemorrhage (291).

\subsubsection{Bacterial Meningitis}

A meta-analysis has shown that corticosteroid use reduces mortality in S. pneumoniae meningitis and decreases the risk of hearing loss in children with $H$. influenzae meningitis; nonetheless, the subgroup analysis determined an increase in the incidence of recurrent fever (295). Based on the current guidelines; dexamethasone is recommended 10 mg every 6 hours for the first 4 days to reduce the risk of neurological sequelae (particularly hearing loss). For the side effects of corticosteroids, 0.15 $\mathrm{mg} / \mathrm{kg}$ dexamethasone can be given every 6 hours for the first 4 days in those with high risk and low body weight. Dexamethasone should be given before or with the first dose of antibiotics (245).

\subsubsection{Tuberculous Meningitis}

A review reported that the mortality was reduced by $25 \%$ in the $3-18$ month follow-up of the patients who used corticosteroids, but the neurological outcomes were not affected (296). Despite the recommendation of corticosteroids to reduce mortality, the current literature is insufficient to make specific recommendations on which corticosteroid to be given at which dose. Corticosteroid therapy should be continued for more than 2 weeks (245).

\subsubsection{Cerebral Venous Thrombosis}

According to one review, more than $90 \%$ of cases with cerebral venous thrombosis due to Behçet's disease received and benefited from corticosteroids (297).

\subsubsection{Brain Tumors}

Vasogenic edema is the most common cerebral edema in brain tumors. The use of corticosteroids is recommended in primary and metastatic brain tumors. Corticosteroids stabilize the impaired blood-brain barrier and reduce capillary permeability. Dexamethasone is most 
commonly used due to its lower mineralocorticoid effect. The peak effect is achieved in 24-72 hours. Corticosteroids provide temporary benefit in symptomatic patients with brain metastases and increased intracranial pressure due to mass effect. The most appropriate corticosteroid choice is dexamethasone at the minimum effective dose. Dexamethasone is preferred 4-8 $\mathrm{mg} /$ day for those with mild symptoms and $16 \mathrm{mg} /$ day for those with moderate-to-severe symptoms (298).

\subsection{Hyperventilation in the Treatment of ICP Increase}

Hyperventilation has been used for many years to reduce increased intracranial pressure (ICP). By definition, it refers to reducing or maintaining the $\mathrm{PaCO}_{2}$ in arterial blood below normal levels by increasing alveolar ventilation.

Constant and adequate maintenance of cerebral blood flow despite physiological and metabolic variability such as blood gases, viscosity, and arterial blood pressure is provided by 'cerebral autoregulation', and dilation or constriction of cerebral arterioles according to these changes plays a primary role in this adaptation. Accordingly, the aim of therapeutic hyperventilation is that the resulting hypocapnia induces vasoconstriction in cerebral arterioles, and this vasoconstriction decreases cerebral blood flow and cerebral blood volume, resulting in a decrease in intracranial pressure $(299,300)$. This vascular activity, called $\mathrm{CO}_{2}$ eactivity, occurs only at values of $\mathrm{PaCO}_{2}$ in the range of $20-60 \mathrm{mmHg}$, and there is no change in vessel diameter at values below or above this $(300,301)$.

Although hyperventilation is a fast and effective method of reducing intracranial pressure, this effect ends in less than 24 hours. Because reactivity to $\mathrm{CO}_{2}$ is dependent on changes in perivascular $\mathrm{pH}$ and alkalosis caused by hypocapnia rapidly activates buffering mechanisms. As a result, CSF and perivascular $\mathrm{pH}$ are normalized, and the effect of hyperventilation decreases within hours $(302,303)$. Therefore, prolonged and deep hyperventilation is not recommended, as it is temporary and may impair cerebral perfusion and increase neurological damage. In addition, since abrupt termination of hyperventilation may result in vasodilation in cerebral arterioles and a related rebound increase in ICP, it should be terminated slowly within 4-6 hours (304).
The crucial point in the application of hyperventilation is to ensure a decrease in ICP while at the same time maintaining normal cerebral blood flow. Hyperventilation-induced reduction in cerebral blood flow can lead to inadequate cerebral perfusion. When $\mathrm{PaCO}_{2}$ drops from the normocapnic level $(\sim 41 \mathrm{mmHg})$ to the hypocapnic level $(\sim 25 \mathrm{mmHg})$ in healthy individuals, approximately a $30 \%$ decrease is observed in cerebral blood flow; albeit only a 7\% decrease in cerebral blood volume. However, the effects of hyperventilation differ in the normal and damaged brain. Although cerebral blood flow decreases in both cases, this decrease is greater in the normal brain than in the damaged brain. This is because hyperventilation causes a redistribution of cerebral blood flow from normal to damaged tissue, consistent with the luxury perfusion in acute cerebral injury $(303,305)$. Studies on this subject have been carried out mostly in traumatic brain injury cases, and some have shown that hyperventilation does not have a significant effect on cerebral oxygenation and metabolism, while others have revealed that it may have adverse effects (306-308). For this reason, hyperventilation should be used together with oxygenation monitoring. $\mathrm{PaCO}_{2}$ or end-tidal $\mathrm{CO}_{2}$ levels in the arterial blood gas should be closely monitored. At the same time, due to the risk of ischemic hypoxia, cerebral oxygenation can be monitored globally by central venous oxygen saturation (Scv02), which can be measured from the jugular vein or locally by the partial oxygen pressure of the brain tissue (pbt02) $(300,309)$.

Hyperventilation, a temporary method to reduce intracranial pressure, can be applied in cases such as acute herniation syndromes and severe ICP elevation with type A plateau waves (221). Hyperventilation should not be used on a chronic basis. Although it may be beneficial in refractory intracerebral hemorrhages and hemorrhages secondary to cerebral hyperemia, its usefulness in hemorrhagic stroke is controversial. In addition, it is not recommended, especially in the first 24-48 hours, as it may impair local cerebral perfusion in traumatic brain injury and acute stroke. Current data in traumatic brain injury suggest that short-term hyperventilation of 15-30 minutes, accompanied by close monitoring, with a target of 30-35 mm $\mathrm{mgCO}_{2}$ and a cerebral perfusion pressure of 60-70 $\mathrm{mmHg}$, may be effective in controlling intracranial hypertension 
(300). Although the evidence on the outcome is insufficient, its use during decompressive craniectomy is also anticipated to be beneficial by increasing the relaxation of the brain (310). In addition, since prophylactic hyperventilation does not prevent ICP increase, prophylactic applications should be avoided if there is no evidence of ICP elevation.

In conclusion, although further studies are necessary on the optimal use of hyperventilation for the treatment of cerebral edema associated with cerebrovascular disease, it is an alternative method that should be kept in mind in a specific patient group in the short-term control of increased resistant ICP. It should be noted that deep and prolonged hyperventilation carries a risk of ischemia and should be used together with multimodal monitoring.

\subsection{Therapeutic Hypothermia and} Targeted Temperature Management in the Treatment of rICP

Thermoregulation is a type of homeostasis and plays a crucial role in survival and maintenance of optimal cellular functions (311). Therapeutic hypothermia is the controlled reduction of body temperature below $32-34 \mathrm{C}^{\circ}$. Therapeutic hypothermia is an effective way to protect organs such as the brain, heart, and kidneys during various surgeries (312), as well as to improve the neurological outcome in cardiac arrest, traumatic brain injury, spinal cord injuries, and increased intracranial pressure following stroke (313).

When ischemia develops in the brain, extracellular glutamate initiates a cascade of neuronal cell death, resulting in increased NMDA response. Therapeutic hypothermia reduces the extracellular levels of excitatory neurotransmitters such as dopamine and glutamate, ensures the integrity and survival of neurons, and reduces the hyperexcitability of the central nervous system (313). It increases the expression of antiapoptotic Bcl-2 by inhibiting caspase activity. It shows anti-apoptotic effects by preventing mitochondrial dysfunction (314). Another neuroprotective mechanism is to reduce free radical formation and consumption of endogenous antioxidants following ischemia (315).

Turkish Journal of Cerebrovascular Diseases 2021; 27(2): 65-132

\subsubsection{Optimum Cooling Methods}

Cooling methods in humans largely involve surface and endovascular cooling. Surface cooling is achieved using ice packs, cooling blankets, or cooling pads. Intravascular cooling is achieved by intravenous infusion of $40 \mathrm{ml} / \mathrm{kg}$ dose of $+4 \mathrm{C} \mathrm{dW}$ Ringer's lactate at a rate of $100 \mathrm{ml} / \mathrm{min}$ or $2000 \mathrm{ml}$ rapid infusion of iced saline. Endovascular cooling is provided by a special heat exchange catheter placed from the femoral vein to the inferior vena cava (316).

\subsubsection{Selective Brain Cooling}

Trans-arterial brain cooling is performed by perfusion of cold perfusate through the ipsilateral carotid artery. This approach can be easily applied in conjunction with endovascular therapies currently in clinical use for the treatment of acute ischemic stroke (317).

\section{Methods}

5.5.3 Hypothermia by Pharmacological

Hipotermi etkinliği olan sekiz farmakolojik ajan vardır. $\mathrm{Bu}$ bileşikler kannabinoid, opioid, transient reseptör potansiyelli vanilloid 1, nörotensin ve tiroksin türevleri, dopamin, xenon ve helyum gibi gazlar ve adenozin türevlerini içermektedir. İlginç bir șekilde kannabinoid grubu ilaçlardan bazıları yalnızca hipotermi indüksiyonu yoluyla nöroproteksiyon sağlamakla kalmaz aynı zamanda ilacın kendisi de farmakolojik mekanizmalar yoluyla nöroproteksiyon sağlayabilmektedir. Bu nedenle, bu ajanlar titreme ve vazokontriksiyonu azaltmada, soğutma sürecini hızlandırmada ve tolere edilebilir soğutma sürelerini uzatmada fiziksel hipotermi ile kombine edilebilmektedir (318).

\subsubsection{Management of Hypothermia}

Today, the term targeted temperature management is used instead of the concept of therapeutic hypothermia. Targeted hypothermia treatment involves determining a target body temperature value below $36^{\circ} \mathrm{C}$ and reaching that temperature quickly. It is carried out in three phases: induction (cooling), maintenance, and rewarming phase. In the induction phase, the target temperature is tried to be achieved within 2-4 hours. Once the target temperature is achieved, the temperature should be maintained 
for 24 hours, and temperature fluctuations should not be allowed. Magnesium can be given as a $4 \mathrm{mg}$ IV bolus for shivering. Anesthesia, analgesia, and neuromuscular blockade are provided by infusing sedo-analgesic agents (propofol is the first choice, if contraindicated midazolam, fentanyl can be used as an analgesic) and neuromuscular junction blocking agents for 24 hours. ECG monitoring of the patients should be performed, and care should be taken against long QT and possible arrhythmias. Patients are followed on a ventilator. Hypoxia or hyperoxygenation should be avoided, and normocapnia should be ensured. Metabolic parameters such as glucose, electrolytes, lactate, and serum $\mathrm{pH}$ should be closely monitored. Infections are common, as hypothermia can compromise the body's ability to fight infections, starting empirical antibiotic therapy is controversial. In the rewarming phase, a warming rate of $0.2-0.5^{\circ} \mathrm{C}$ per hour is recommended according to the 2015 ERC guideline. Hyperthermia ( $>37.5 \mathrm{C}$ ) should not be allowed for 72 hours, as rebound hyperthermia may increase neurological damage (319).

There is no evidence in the literature showing the superiority of any cooling method. PIn practice, a combination of surface cooling and intravascular cooling is often used to achieve desired temperatures (320). The 2020 guidelines do not recommend the rapid infusion of cold IV fluids in the prehospital setting. Many institutional protocols aim to reach the target temperature within 4-6 hours (321).

Although it has been demonstrated in animal models of acute ischemic stroke that therapeutic hypothermia reduces cerebral metabolic demand and decreases brain edema and infarct volume (322), the effect of therapeutic hypothermia is still controversial in randomized controlled trials in patients with acute ischemic stroke. In the metaanalysis collecting the results of 8 randomized controlled and 4 prospective observational cohort studies conducted between 2000 and 2019, therapeutic hypothermia application slightly contributed to achieving functional independence, defined as mRS $0-2$, but did not have a statistically significant effect. A greater improvement was shown in patients with superficial cooling of only the head and neck region and in patients cooled for more than 48 hours. Symptomatic intracranial hemorrhage, hemorrhagic transformation, deep vein thrombosis, and pneumonia were higher in the therapeutic hypothermia group, the highest being cardiac complications (323). Studies demonstrating the efficacy of therapeutic hypothermia in cerebral hemorrhage are more limited. While therapeutic hypothermia does not cause a significant decrease in poor clinical outcome and mortality, care should be taken for preventable complications such as rebleeding, pneumonia, sepsis, arrhythmia, and hydrocephalus (324).

In conclusion, although the neuroprotective effects of targeted temperature management have been shown in experimental models, the efficacy and safety of this treatment should be demonstrated by randomized clinical studies with a large number of patients.

\subsubsection{Side Effects of Therapeutic Hypothermia}

It can cause an increase in infection risk, pneumonia, and sepsis due to impaired platelet aggregation, coagulopathy, impaired tissue healing due to vasoconstriction, and impaired immune cell function (325). A decrease in potassium, magnesium, and phosphate levels and deterioration in insulin secretion and immune system can be observed (326). Arrhythmias such as reflex diuresis (327), systemic hypotension, pulmonary embolism, deep vein thrombosis, seizure, bradycardia, prolongation of the QT interval, and ventricular fibrillation/tachycardia can be observed (328).

\subsection{External Ventricular Drainage in the Treatment of rICP}

External ventricular drainage (EVD) has been used to relieve hydrocephalus for over a century. Cerebrospinal fluid (CSF) drainage is effective in reducing intracranial pressure as well as improving cerebral perfusion pressure, cerebral oxygenation, and metabolism (329).

\subsubsection{Indications}

Acute hydrocephalus, subarachnoid hemorrhage (SAH), intraventricular hemorrhage (IVH), intraparenchymal hemorrhage (IPH), meningitis, brain tumors, infusing intraventricular drugs, and ventriculoperitoneal shunt failure are the most common indications for $\operatorname{EVD}(330,331)$. EVDs are also included in the "Brain Trauma Foundation" guideline for the treatment of severe traumatic brain injury (TBI) (332). 
CSF drainage is a method of reducing the pressure in ICP. The advantage is that EVD can both diagnose and treat high intracranial pressure (331). A study comparing EVD and intraparenchymal devices measuring only intracranial pressure in patients with severe traumatic brain injury has shown that EVD is associated with a lower incidence of in-hospital mortality and better functional and neurocognitive outcomes 6 months after injury (333).

\subsubsection{EVD Placement}

The ideal location for EVD placement is the frontal horn of the right lateral ventricle, close to the foramen of Monro (334). When a right frontal placement is not possible due to intracranial pathological findings, the left frontal region can be considered.

The EVD entry point is located in the skull approximately $11-12 \mathrm{~cm}$ above the nasion or $2-3$ $\mathrm{cm}$ in front of the coronal suture and $2-3 \mathrm{~cm}$ to the right of the midline and roughly corresponds to the mid-pupillary line. This entry point ensures that the EVD does not damage the superior sagittal sinus or motor cortex. CSF flow is controlled by slowly advancing the catheter to a depth of $6.5 \mathrm{~cm}$ (331).

During EVD placement, care should be taken to apply aseptic techniques (sterile gloves, sterile gown, hood, and mask), administer a single dose of antibiotics 15-45 minutes before the incision (e.g., cefazolin), cut hair, and wash the area with chlorhexidine (334).

EVD placement should be avoided in patients taking anticoagulant or antiplatelet drugs and in patients with coagulopathy (platelet count less than 100,000 per cubic millimeter). EVD catheters should not be placed near the infected area of the scalp (331).

\subsubsection{Complications}

Complications include intracranial bleeding, infection (catheter-associated ventriculitis, meningitis, brain abscess, and subdural empyema), catheter mispositioning, and catheter occlusion.

The incidence of bleeding was reported to be between 0-41\% (335-337).

Brain computed tomography (BCT) may be beneficial after EVD insertion to control the position of the catheter and to exclude intracranial hemorrhage.
It is recommended to give a single dose of antibiotics before the use of EVD (333). Antibiotic use is not recommended as long as the EVD remains. Using antibiotic-coated EVD catheters, tunneling the catheters, not changing the catheters routinely, obtaining a CSF sample only if clinical ventriculitis is suspected, and limiting the EVD drainage time are recommended to reduce the infection rate $(338,339)$.

Clamping of EVD is recommended to prevent excessive drainage of CSF during intrahospital transport (340). Intracranial hypertension associated with EVD clamping may occur during transport (341).

\subsubsection{Removal of EVD}

In traumatic brain injury, the EVD weaning trial usually takes place within 7-14 days after the first injury. Because in this period, the risk of rebleeding and cerebral edema decreases (342).

\subsection{Sedation Applications in rICP}

\subsubsection{Propofol}

One of the methods used to reduce intracranial pressure is the use of sedative drugs. Propofol is one of the most commonly used sedative drugs in practice. Propofol (2,6diisopropyl phenol) is a sedative-hypnotic drug that has been widely used since 1989 for the induction and maintenance of sedation or general anesthesia $(343,344)$. Propofol is thought to act by enhancing the binding of gamma-aminobutyric acid to receptor sites in the central nervous system. It also binds antagonistically to N-methyl $\mathrm{D}$-aspartate (NMDA) receptors (345). Propofol is the drug of choice in Neurology Intensive Care Units, with a rapid onset time of approximately 3060 seconds, a short half-life of 10-15 minutes, and a rapid wake-up time after drug discontinuation $(346,347)$. Its clearance is independent of renal and hepatic functions. Propofol is commercially available in $1 \%$ and $2 \%$ emulsions. It is available in $20 \mathrm{ml}$ ampoules, 50 and $100 \mathrm{ml}$ vials. 1\% solutions contain $10 \mathrm{mg}$ of propofol in $1 \mathrm{ml}$, and $2 \%$ preparations contain $20 \mathrm{mg}$ of propofol in $1 \mathrm{ml}$. An induction dose of $0.5-1 \mathrm{mg} / \mathrm{kg}$ is needed for general anesthesia. In the intensive care unit, the starting dose is $5 \mathrm{mcg} / \mathrm{kg} /$ minute $(0.3 \mathrm{mg} / \mathrm{kg}$ per hour), and the infusion dose can be increased by titration with $5-10 \mathrm{mcg} / \mathrm{kg} / \mathrm{min}$ increments at intervals of at least 5 minutes up to $1-3 \mathrm{mg} / \mathrm{kg}$ per hour depending on the desired clinical efficacy. 
Due to its short duration of action, it is possible to perform a rapid neurological evaluation by reducing the dose while the patient is being monitored on the ventilator. Concomitant use with midazolam infusion (0.02-0.1 $\mathrm{mg} / \mathrm{kg}$ per hour) provides more comfortable hemodynamic stabilization, quicker weaning from the ventilator, and lower doses of both drugs. In order to provide analgesia, fentanyl infusion (25-250 mcg/hour) can be made together with propofol.

Propofol reduces the cerebral metabolic rate of oxygen (CMRO2). It leads to a parallel decrease in cerebral blood flow and cerebral blood volume. This decrease in cerebral blood volume causes a decrease in intracranial volume and hence in ICP. It can also lower mean blood pressure and cerebral perfusion pressure, especially at high doses. During propofol infusion, heart rate, cardiac output, systemic vascular resistance, and central venous pressure also decrease, and the consequent hypotension can be a problem, especially in hypovolemic patients. It is safer than barbiturates in terms of cardiovascular hemodynamics. Since it suppresses the respiratory response to hypercapnia and reduces upper airway reflexes and reactive bronchodilation, it can increase $\mathrm{CO}_{2}$ production. In a mechanical ventilator, it is necessary to increase the respiratory rate per minute $(348,349)$.

The disadvantages of propofol are that it does not have an analgesic effect, does not cause amnesia, especially at low doses, develops tachyphylaxis and tolerance, increasing triglyceride level, slows down the metabolic rate and increasing the calorie intake relatively, and causing propofol infusion syndrome. Propofol infusion syndrome is a rare but potentially fatal complication characterized by increased lactic acid and severe metabolic acidosis, rhabdomyolysis, renal failure, and cardiocirculatory shock after high doses of propofol ( $>4 \mathrm{mg} / \mathrm{kg} /$ hour) and longterm use ( $>48$ hours) (350).

\subsubsection{Pentothal Coma}

Increased intracranial pressure syndrome is a condition that needs urgent treatment as it can lead to secondary brain damage and death (351). Barbiturate coma is one of the last-line treatments in patients whose refractory ICP persists despite all pharmacological and non-pharmacological treatments. Barbiturates depress the reticular activating system by slowing the rate at which the inhibitory neurotransmitter GABA leaves its receptors and by stimulating the inhibitory AMPA receptors. They are sedative/hypnotic, anticonvulsant drugs commonly used for anesthesia and epilepsy, but they do not have analgesic effects. In our country, $0.5 \mathrm{gr}$ and $1 \mathrm{gr}$ vials of pentothal are only suitable for intravenous use. Pentothal decreases cerebral blood flow, cerebral oxygen metabolism rate, and intracranial pressure and increases the epileptic seizure threshold. Its effects on cerebral perfusion pressure are variable, as it also has the ability to reduce mean arterial pressure (352). Although its mechanism and efficacy are not entirely clear, while the metabolism slows down and blood flow decreases in the whole brain, it improves blood flow in the ischemic area by maximally dilating the vessels in focal ischemia areas (reverse "Robin Hood" phenomenon) (353). The hypnotic effect starts in 15-20 seconds after intravenous administration and lasts 5-10 minutes. It accumulates in adipose tissue at a concentration approximately 6-12 times higher than in plasma. When applied in repeated doses, it causes longterm anesthesia as it continues to be released slowly from the adipose tissue. Its half-life is 3-8 hours. It is metabolized primarily in the liver and to a smaller extent in the brain and kidney. The biotransformation product is pharmacologically inactive and is mostly excreted in the urine. As the dose is increased, the plasma concentration becomes very high (zero-order kinetics). Although the dose required to induce medical coma in patients with ICP varies according to the patient, the general recommendation is to administer Pentothal by titration at a dose of $1 \mathrm{mg} / \mathrm{kg} /$ hour after 5-10 mg/kg loading dose. If EEG monitoring can be performed on the patient, the ideal dose of pentothal should be determined according to the "burst suppression" pattern in EEG. In this case, the EEG should show an isoelectric line for about 5-10 seconds and then a trace that shows the activity as a burst for 5-10 seconds (354). Pentothal is in the last-line treatment of rICP because it has many undesirable side effects. Pentothal directly suppresses the cardiac contraction force, reduces venous tone, causes blood to pool in the periphery, and may cause severe hypotension, especially in hypovolemic patients. Most of the patients who are started pentothal will need inotropes in a short time. Multiple organ failure, acidosis, and shock are 
observed frequently in patients receiving inotropic support in addition to pentothal for a long time. Pentothal reduces the overall systemic metabolic rate and the gastrointestinal muscle tone and may cause severe enteral intolerance in patients. In patients with pentothal coma, parenteral nutrition may be preferred instead of enteral nutrition. Other side effects during the infusion are hypokalemia (rebound hyperkalemia following cessation of infusion), bronchospasm, laryngospasm, respiratory depression, hypothermia, and immunosuppression. Its use is contraindicated in patients with asthma or porphyria. One of the most important effects is that pentothal accumulated in the adipose tissue continues to be released after the cessation of the infusion, and the neurological evaluation of the patients cannot be performed due to its prolonged effect. It may be more reliable to refer to a supportive test that evaluates cerebral blood circulation for a definitive diagnosis, especially if barbiturate use is considered in patients followed up for brain death. Indeed, many centers reported that the evaluation of brain stem reflexes was delayed in patients who received pentothal infusion, and different algorithms were used for brain death determination (355). Routine barbiturate use is not recommended in edematous brain infarcts, as there is insufficient data in the 2014 AHA/ASA recommendations (class 3) (356). Barbiturate coma is an alternative practice to be considered in refractory rICP patients. Pentothal, on the other hand, can be preferred due to its short duration of action. Mechanically ventilated patients should be followed closely for hemodynamic and gastrointestinal problems and infections, and electrolyte levels should be checked at frequent intervals. It should be noted that the effect may continue for a long time due to redistribution even after the cessation of the drug hence continuous EEG monitoring is recommended for the optimal dose. Although our primary goal is to protect brain functions, it is necessary to consider the patient as a whole and choose the treatment according to the benefit and harm balance.

\subsection{Decompressive Surgery in Malignant Middle Cerebral Artery Infarction}

Conventional applications and medical treatments may be insufficient to prevent and treat cerebral edema and increased intracranial pressure in large MCA infarcts. At this stage, decompressive surgery can be life-saving in suitable patients. Decompressive surgery aims to expand the edematous ischemic brain tissue outward by removing part of the cranium bone on the affected side and opening the dura mater and thereby prevent secondary damages due to intracranial edema. The return of intracranial pressure to normal ensures an increase in cerebral blood flow, improvement in cerebral perfusion pressure, and better oxygenation in the still healthy brain tissue (282).

Decompression surgery was first applied to a patient with post-traumatic brain injury by Kocher in 1901 and to a patient with a brain tumor by Cushing in $1905 \quad(357,358)$. With the improvement in its usage area and technique over the years, the method started to be applied in the treatment of large cerebral infarcts for the first time in the 1970s $(359,360)$. Today, the most commonly used method in malignant MCA infarcts is frontotemporoparietal craniectomy. Since the surgery will be performed in a large area, the patient should be positioned such that a wide area of the scalp can be dominated, and care should be taken not to disrupt the jugular vein flow during the positioning of the patient's head to avoid a further increase in the intracranial pressure. The skin incision should be approximately $1 \mathrm{~cm}$ larger than the planned craniectomy area for convenience when enlarging the surgical field during the procedure or reopening the skin when cranioplasty is required $(361,362)$. The bone flap must be removed in a sufficiently large size to prevent cerebral herniation and to ensure cerebral perfusion without ischemia. The anteriorposterior diameter of the craniectomy area should be at least $12 \mathrm{~cm}$ in malignant MCA infarcts (363). There may be a severe outward herniation from an undersized craniectomy area, resulting in compression of the cerebral veins at the bony margins, new areas of bleeding, and cortical damage (364). In addition, the dura must be opened wide enough to give enough space to the cerebral tissue. The bone flap removed during surgery can either be placed in subcutaneous fat tissue in the patient's abdomen for later use or stored in a bone bank at low temperature $\left(-80^{\circ} \mathrm{C}\right.$ and below) (365). Depending on the patient's neurologic recovery status, the removed bone tissue can be replaced by a second procedure with the patient's own bone flap (autograft) or allograft materials. There is no clear timetable in the literature on when to perform cranioplasty, but performing cranioplasty within 2 months after 
decompressive surgery has been indicated to possess a higher risk of complications. For this reason, the recommended is to wait 4-6 months for bone graft replacement (366, 367). Decompressive surgery can have early (first 4 weeks) and late ( $>4$ weeks) complications. While complications may be related to the surgical procedure, the risk of developing complications is higher, especially in patients over 65 years of age and patients with a Glasgow Coma Score below 8 . Early complications are bleeding, extracranial cerebral herniation, paradoxical herniation, wound complications, CSF leak, postoperative infections, and epileptic seizures. Late complications are subdural effusion, hydrocephalus, and Trephine syndrome (368). Existing contusion or bleeding on the lesion side may increase after craniectomy. Intracranial hemorrhages may also occur on the noncraniectomy side. Post-surgical control CT should be seen for bleeding control, especially in the first 24 or 48 hours. An extracranial cerebral herniation is one of the possible early complications, especially during the first week. Outward herniation of cerebral tissue due to brain edema may induce ischemia or parenchymal lacerations caused by venous congestion developing after compression of the drainage veins at the corners of craniectomy (369). In order to prevent this complication, a sufficiently wide flap must be removed. Paradoxical herniation, on the other hand, is a rare complication that can develop due to negative subatmospheric pressure. It is the displacement of the midline towards the intact hemisphere. Patients with paradoxical herniation should be followed in the Trendelenburg position with ample intravenous hydration. The frequency of epileptic seizures after decompressive surgery was determined as high as $50 \%$ in some publications (370). Wound complications related to incision are usually caused by ischemia and necrosis following the obstruction of the flow in the superficial temporal artery. CSF leak is a complication that requires early intervention as it increases the risk of infection. Post-operative superficial wound infection, subgaleal collections, as well as mortality-increasing infections such as epidural or subdural abscess can be seen (371). Subdural effusion results from altered CSF circulation due to decreased intracranial pressure after decompressive surgery (372). Another late complication is hydrocephalus. Advanced age, subarachnoid hemorrhage, CSF infection, and excessively large craniectomy increase the risk of hydrocephalus. Trephine Syndrome is a late complication that develops with atmospheric pressure changes, especially in large craniectomies, with headache, restlessness, weakness, and psychiatric problems. The risk of both hydrocephalus and Trephine syndrome can be reduced by early cranioplasty.

The concern regarding whether decompressive surgery should be applied to patients followed up with malignant MCA infarction, and if so, to which patients and when has always led to confusion $(362,367)$. Eight randomized controlled studies and meta-analyses published in the last 20 years define our clinical approach. Although the design, inclusion criteria, and endpoints of these eight randomized controlled trials were different, they all compared patients undergoing surgery and patients treated with medical therapy alone (373-380). The common endpoint for most of them was mortality and functional outcome at 6 and 12 months. Only patients under 60 years of age were included in three studies (DECIMAL, DESTINY, HAMLET). Six studies included patients with pre-stroke modified Rankin Scale (mRS) 0-1, while two studies (HeADDFIRST, HeMMI) included patients with mRS 0-2 (Table 5).

Mortality: Mortality was determined as the primary or secondary endpoint in most of the studies. There was no difference in mortality between the two groups in only two studies (HeADDFIRST, HeMMI), while in the other six studies, mortality was lower in the surgical group. In the DECIMAL study, 6-month mortality was lower in the surgery group $(25 \%-78.2 \%$ $\mathrm{p}<0.0001$ ), and the study was terminated early because there was a significant mortality difference between the two groups (373). In the DESTINY study, survival at 1 month was significantly higher in the surgery group (88\%$47 \% \mathrm{p}=0.02$ ) (374). In the HAMLET study, 12month mortality was lower in the surgery group (22\%-59\% p=0.002) (375). The study conducted by Sleinz et al. in Lithuania in 2000 on 24 patients determined the survival rate as $45.5 \%$ (5 patients) in the surgery group and as $7.69 \%$ (1 patient) in the medically followed group $(p=0.06)$ (376). The study of Zhao et al. on 47 patients aged 18-80 determined that 6 th and 12 th-month survival was significantly lower in the decompressive surgery 
group $(12.5 \%-60.9 \% \mathrm{p}=001,16.7 \%-69.6 \% \mathrm{p}$ $<0.001$, respectively) (377). In the other two studies conducted on 24 patients (HeADDFIRST and HeMMI), there was no statistically significant difference between mortality rates, and this was attributed to the low number of patients. In the HeADDFIRST study, there is no statistically significant difference between the 21st and the 180th-day mortality rates (surgical group 21\% medical group $21 \% \mathrm{P}=0.39$; surgical group $36 \%$ medical group 40\%, respectively) (378). YiAgain in the HeMMI study, the difference between the 6thmonth mortality rates of the 2 groups was not statistically significant (380). The DESTINY 2 study of patients above 60 years of age was planned to evaluate the effectiveness of decompressive surgery in advanced age patients (mean age 70), and both 6-month and 12-month mortality rates were found lower in the surgical group (33\%-70\%, $43 \%$ - 76\%, respectively) (379). Meta-analyses covering all these randomized controlled studies have also shown that mortality is lower in patients with decompressive surgery (381-386).

Functional Outcome: Although mRS $0-3$ is accepted as a good functional outcome in most stroke studies, mRS $0-4$ has been accepted as a good functional outcome criterion in some studies on decompressive surgery, considering the severity of the disease.

In the DECIMAL, DESTINY, and HeMMI studies, the primary endpoint was defined as a good functional outcome (mRS 0-3) at the 6month. In the DECIMAL study, no statistically significant difference was found between the two groups in the number of patients with mRS 0-3 at 6 months and 12 months (25\%-5.6\% p =0.18; $50 \%-22.8 \% \mathrm{p}=1$ in surgical and medical groups, respectively). However, analyzes in the patient group with mRS 0-4 at 12 months revealed better outcomes in the surgical group (75\%-22.2\% $\mathrm{p}=0.0029$ ) (373). Similarly, there was no significant difference between the two groups in the DESTINY study in patients with mRS 0-3 $(\mathrm{p}=0.23)$, but analyses according to mRS $0-4$ revealed an improvement in favor of the surgical group (77\%-33\%, p=0.01) (374). In the HeMMI study, comparisons of both mRS 0-3 and mRS 0-4 patients showed no significant difference between the two groups. In the study by Zhao et al. and in the DESTINY 2 study, the 6th-month mRS was determined as 0-4 for a good functional outcome and better results were obtained in the surgery group in both studies $(377,379)$. In the HAMLET study, patients with mRS 0-3 at 12 months were evaluated for a good functional outcome and no significant difference was found between the two groups (375).

Except for 1 patient in the HeADDFIRST study, none of the 7 randomized controlled trials (not all mRSs were given in the HeMMI study) were identified as mRS $0-1$. In the pooled analysis of the DESTINY, DECIMAL, and HAMLET studies, an absolute risk reduction was found in the surgical group at 1 year in both the mRS 0-3 and mRS $0-4$ groups $(23 \%, 51 \%$, respectively) (381). In the meta-analysis of 7 randomized controlled studies published by Alexander et al. in 2016, evaluations with both mRS 0-3 and mRS 0-4 demonstrated a significant increase in the good outcome in the surgical group ( $\mathrm{p}=0.04, \mathrm{p}<0.0001)$ (384). In the meta-analysis published by Reinin et al. in 2000, the number of patients with mRS 0-3 at 12 months was also higher in the surgical group (37\% - 15\%, p<0.001) (386).

Age: Studies have shown that the age of the patient has a significant effect on the results of decompressive surgery performed in large MCA infarcts (387). Strong evidence supports that decompressive surgery is a life-saving intervention in patients under 60 years of age with malignant MCA infarction. However, today, neurologists and neurosurgeons still discuss the age up to which decompressive surgery can be performed in patients with malignant MCA infarction. A review published by Gupta et al. in 2000, in which 138 patients who underwent decompressive surgery due to malignant MCA infarction were examined, reported that $80 \%$ of 75 patients over 50 years of age and $32 \%$ of 63 patients under 50 years of age had mRS 4-6 (388). In randomized controlled studies, patients over the age of 60 were included in 5 of the 8 studies, but the mean age was still below 65 in the others, except for the DESTINY 2 study (376-380). In the DESTINY 2 study, the mean age is 70 (379). In the first 3 RCSs (DECIMAL, DESTINY, and HAMLET), the mean age is below 50 years (373-375). There is also no upper age limit in the study by Slezins et al. and the DESTINY 2 study. While the number of survivors over 60 years of age was not specified in the HeADDFIRST and HeMMI studies, the study by Slezins et al. reported that there were no survivors above 60 years of age in the surgical group, and 
the only survivor in the medical treatment group was 72 years old $(376,378,380)$. The DESTINY 2 study showed that decompressive surgery also reduced mortality in patients over 60 years of age, and statistically significant results in favor of surgery were also obtained with a primary endpoint of mRS $0-4$ at 6 months (38\%-18\%, $\mathrm{p}=0.04$ ). However, considering that patients with mRS 4 have severe disability and cannot be mobilized without support, it is seen that the results between the two groups are not significant when this comparison is made with an outcome defined as mRS 0-3. Although deaths were less common in the surgical group $(33 \%-70 \%)$, the number of both mRS 4 and mRS 5 patients was higher in the surgical group (32\%-15\%, 28\%-13\%, respectively) (379). In line with these results, if possible, patients over the age of 60 or their relatives should be informed before surgery that surgery reduces mortality, but the risk of survivors needing care is very high.

Timing: Brain edema occurring in malignant MCA infarcts can cause herniation and death with a space-occupying effect. The important thing here is that the patients are diagnosed before irreversible damage develops and the herniation clinic advances and are referred for decompressive surgery. The pooled analysis of the DECIMAL, DESTINY, and HAMLET studies has been shown that decompressive surgery performed both in the first 24 hours and between 24-48 hours reduces mortality and increases the good outcome (381). Among these 3 studies, patients over 48 hours were included only in the HAMLET study (DECIMAL <24 hours, DESTINY 1236 hours, HAMLET 96 hours). In the subgroup analysis of the HAMLET study, comparison of the patients who underwent surgery within 48 hours and those who underwent surgery after 48 hours determined that mortality and good outcome were in favor of surgery in the first group, whereas surgery was not beneficial in the group operated after 48 hours (375). In a large retrospective analysis published by Dasenbrock et al. in 2017, 1301 patients who underwent decompressive surgery for ischemic stroke were examined. In terms of poor outcome (death, tracheostomy, gastrostomy, discharge to the care center), there was no difference between patients operated on within 24 and 48 hours, however, patients operated on after 72 hours had a higher rate of poor outcome (74.9\% - 68.7\%, p=0.02) (389).
A prophylactic approach to be applied too early may lead to an excessive patient referral for surgery or may expose the patient to a surgical procedure with a higher complication rate while medical treatment may be sufficient. On the contrary, if it is waited for the patient to progress clinically to undergo surgery, this time the patient may not benefit from the surgery due to the development of irreversible damage. Therefore, as emphasized before, it would be appropriate to decide on the timing of surgery by considering the clinical and radiological prognostic factors for patients. Considering the recommendations of different associations and institutions regarding decompressive surgery, decompressive surgery recommendations in malignant MCA infarcts can be listed as follows (389).

$\checkmark$ Decompressive hemicraniectomy is recommended to improve survival, especially in large hemispheric strokes under 60 years of age (strong recommendation, high level of evidence).

$\checkmark$ Decompressive hemicraniectomy may reduce mortality in patients over 60 years of age, but patients and their families should be consulted due to the high risk of severe disability (strong recommendation, moderate level of evidence).

$\checkmark$ For best neurological clinical outcome, decompressive hemicraniectomy should be performed within the first 24-48 hours of symptom onset and before symptoms of herniation develop (strong recommendation, moderate level of evidence).

$\checkmark$ To achieve an optimum decompressive effect, the size of the craniectomy should be at least $12 \mathrm{~cm}$. Bone flaps 14-16 cm wide appear to be associated with a better outcome (strong recommendation, moderate level of evidence).

$\checkmark$ It is recommended to open the dura during decompressive surgery (strong recommendation, moderate level of evidence).

$\checkmark \quad$ The physician should inform the patients and their relatives that there may be severe disability after surgery, and the quality of life may not improve (grade of recommendation based on clinical experience). 
Güngör et al.

Table 5. Summary of 8 randomized controlled trials comparing decompressive surgery with medical therapy in malignant MCA infarcts.

\begin{tabular}{|c|c|c|c|c|c|c|c|c|}
\hline Studies & $\begin{array}{c}\begin{array}{c}\text { Number of } \\
\text { patients } \\
\text { (surgery/medical) }\end{array} \\
\end{array}$ & Age & $\begin{array}{c}\text { Time } \\
\text { (hours) }\end{array}$ & NIHSS /GCS & Imaging & $\begin{array}{l}\text { Prestroke } \\
\quad \text { mRS }\end{array}$ & Outcome & Results \\
\hline $\begin{array}{l}\text { DECIMAL (373) } \\
\text { (2007, France) }\end{array}$ & $38(20 / 18)$ & $18-55$ & $<24$ & $>15$; NIHSS $1 \mathrm{a} \geq 1$ & $\begin{array}{l}\text { Brain CT ischemia: > 50\% MCA } \\
\text { territory } \\
\text { MRI infarct volume: }>145 \mathrm{~cm}^{3}\end{array}$ & $0-1$ & 6-month mRS 0-3 & $\begin{array}{l}\text { 6-month mRS 0-3 no } \\
\text { difference } \\
\text { 6-month mortality lower in } \\
\text { the surgical group }\end{array}$ \\
\hline $\begin{array}{l}\text { DESTINY (374) } \\
(2007, \text { Germany) }\end{array}$ & $32(17 / 15)$ & $18-60$ & $12-36$ & $\begin{array}{c}\text { Right hemispheric lesion }>18 \text {; left } \\
\text { hemispheric lesion }>20 \\
\text { NIHSS } 1 \mathrm{a} \geq 1\end{array}$ & $\begin{array}{c}\text { Brain CT ischemia } \geq 2 / 3 \mathrm{MCA} \\
\text { territory } \\
\text { (including basal ganglia) }\end{array}$ & $0-1$ & 6-month mRS 0-3 and & $\begin{array}{l}\text { 6-month mRS 0-3 no } \\
\text { difference } \\
\text { 6-month mortality lower in } \\
\text { the surgical group }\end{array}$ \\
\hline $\begin{array}{l}\text { HAMLET (375) } \\
\text { (2009, } \\
\text { Netherlands) }\end{array}$ & $64(32 / 32)$ & $18-60$ & $<96$ & $\begin{array}{l}\text { Right hemispheric lesion }>15 \text {; left } \\
\text { hemispheric lesion }>20\end{array}$ & $\begin{array}{c}\text { Brain CT ischemia } \geq 2 / 3 \mathrm{MCA} \\
\text { territory }\end{array}$ & $0-1$ & $\begin{array}{l}\text { 12-month mRS 0-3 } \\
\text { and 4-6 }\end{array}$ & $\begin{array}{l}\text { 12-month mRS 4-6 no } \\
\text { difference } \\
\text { 12-month mortality lower } \\
\text { in the surgical group }\end{array}$ \\
\hline $\begin{array}{l}\text { Slezins et al.(376) } \\
\text { (2012, Latvia) } \\
\text { (Single-centered) }\end{array}$ & $\begin{array}{c}24 \\
(11 / 13)\end{array}$ & $>18$ & $<48$ & $>15$ & $\begin{array}{l}\text { Ischemia on brain CT or MRI: > } \\
50 \% \text { MCA territory or }>145 \mathrm{~cm}^{3} \\
\text { infarct }\end{array}$ & $0-1$ & $\begin{array}{l}\text { 12-month mRS } 0-4 \\
\text { and 5-6 }\end{array}$ & $\begin{array}{l}\text { Mortality lower in the } \\
\text { surgical group }\end{array}$ \\
\hline $\begin{array}{l}\text { Zhao et al. (377) } \\
(2012 \text {, China) }\end{array}$ & $47(24 / 23)$ & $18-80$ & $<48$ & $\mathrm{GCS} \leq 9$ & $\begin{array}{c}\text { Brain CT ischemia } \geq 2 / 3 \mathrm{MCA} \\
\text { territory and space-occupying } \\
\text { edema }\end{array}$ & $0-1$ & $\begin{array}{l}\text { 6-month mRS } 0-4 \text { and } \\
5-6\end{array}$ & $\begin{array}{l}\text { 6-month mRS 5-6 and } \\
\text { mortality lower in the } \\
\text { surgical group }\end{array}$ \\
\hline $\begin{array}{l}\text { HeADDFIRST (378) } \\
(2014, \text { USA })\end{array}$ & $24(14 / 10)$ & $18-75$ & $<96$ & $>17$; NIHSS $1 \mathrm{a}<2$ & $\begin{array}{c}\text { Brain CT ischemia: }>50 \% \text { MCA } \\
\text { territory in first } 5 \text { hours or } \\
\text { complete MCA territory within } 48 \\
\text { hours; and shift }>7.5 \mathrm{~mm} \text { in } \\
\text { septum pellucidum } \\
\text { or }>4 \text { mm in the pineal gland with } \\
\text { NIHSS } 1 \mathrm{a} \geq 2\end{array}$ & $0-2$ & 21-day mortality & $\begin{array}{l}\text { No difference in } 21 \text {-day } \\
\text { mortality in the surgical } \\
\text { group }\end{array}$ \\
\hline $\begin{array}{l}\text { DESTINY } 2 \text { (379) } \\
\text { (2014, Germany) }\end{array}$ & $112(49 / 63)$ & $>60$ & $<48$ & $\begin{array}{c}\text { Right hemispheric lesion }>14 \text {; left } \\
\text { hemispheric lesion }>19 \\
\text { NIHSS } 1 \mathrm{a} \geq 1\end{array}$ & $\begin{array}{l}\geq 2 / 3 \text { MCA territory } \\
\text { (including basal ganglia) }\end{array}$ & $0-1$ & 6-month mRS 0-4 & $\begin{array}{l}\text { 6-month mRS 0-4 higher, } \\
\text { mortality lower in the } \\
\text { surgical group }\end{array}$ \\
\hline $\begin{array}{l}\text { HeMMI (380) } \\
\text { (2015, } \\
\text { Philippines) } \\
\text { (Single-centered) } \\
\end{array}$ & $29(16 / 13)$ & $18-65$ & $<72$ & $\begin{array}{c}\text { GCS; GCS } 15 \text { with right } \\
\text { hemispheric lesion } 6-14, \text { left } \\
\text { hemispheric lesion } 5-9 \text { or NIHSS } \\
1 \mathrm{a} \geq 1 \text { deterioration }\end{array}$ & $\begin{array}{c}\text { Brain CT ischemia: > 50\% MCA } \\
\text { territory }\end{array}$ & $0-2$ & $\begin{array}{l}\text { 6-month mRS } 0-3 \text { and } \\
4-6\end{array}$ & $\begin{array}{l}\text { 6-month mRS } 0-3 \text { and } \\
\text { mortality not different in } \\
\text { the surgical group }\end{array}$ \\
\hline
\end{tabular}

NIHSS: National Institutes of Health Stroke Scale; GCS: Glasgow Coma Scale; mRS: Modified Rankin Scale; CT: Computed Tomography; MRI: Magnetic Resonance Imaging; MCA: Middle Cerebral Artery.

Turkish Journal of Cerebrovascular Diseases 2021; 27(2): 65-132 


\section{Stroke \\ 6.2 Posterior Decompression in Ischemic}

Large cerebellar infarcts often become edematous, and if not treated appropriately and quickly, they can cause coma and death by compressing the 4th ventricle within the restricted area of the posterior fossa and brainstem (390). Acute cerebellar infarcts can cause rapid worsening even days later (391). Large-volume cerebellar infarcts with mass effect should be followed closely, preferably in the intensive care unit; development of impaired consciousness, change in respiratory pattern, a new oculomotor sign, or pyramidal findings such as Babinski sign requires immediate repetition of neuroimaging (390). Cerebellar edema, a life-threatening condition that causes brain stem compression and obstructive hydrocephalus, develops in $10-20 \%$ of patients (392). According to the AHA guideline updated in 2019, when neurologic deterioration due to brainstem compression develops despite maximal medical treatment after cerebellar infarction, decompressive suboccipital craniectomy including dura opening should be performed, and concomitant ventriculostomy is recommended for the treatment of obstructive hydrocephalus. It should be noted that ventriculostomy carries the risk of upper herniation in hydrocephalus caused by infarcts with cerebellar mass effect, hence it should be performed together with posterior craniectomy, or CSF drainage should be very conservative (Class 1 evidence level B-NR) (393).

While there is evidence that surgical intervention can save most patients in cerebellar infarcts that are likely to progress to infratentorial herniation, there are differing opinions regarding patient selection criteria, type of surgery (such as ventriculostomy, posterior fossa craniotomy), and timing of the procedure (394). The lack of large multi-centered randomized clinical trials on this issue is probably due to the frightening clinical course of brain stem compression and hydrocephalus (395). The literature has shown that most cerebellar infarct patients who underwent surgical treatment had posterior inferior cerebellar artery (PICA) territory infarction $(391,396)$. It should be noted that external ventricular drainage (EVD), when applied alone, may pose a risk by causing upper herniation and will not eliminate the brainstem compression
(397). In GASCIS (German-Austrian SpaceOccupying Cerebellar Infarction Study), one of the largest studies in the literature, 84 patients with large cerebellar infarction were prospectively analyzed clinically and radiologically, but the patients were not randomized; 34 of the patients had decompression surgery, 14 of them had ventriculostomy, and 36 patients received the best medical treatment (394). In this study, clinical worsening developed between 2-4 days, and surgical treatment was performed after an average of 62 hours. Since all comatose patients underwent surgery, a comparison could not be made in this group, and there was no significant difference between the treatments in terms of endpoints in patients with clouding of consciousness and somnolence/stupor (394). In a retrospective matched case-control study, preventive suboccipital surgery and the absence of brainstem infarction were associated with a good clinical outcome in patients with cerebellar infarction (397). A concomitant EVD was inserted in $50 \%$ of the patients who underwent suboccipital decompression; infarct tissue resection was also performed in $57 \%(397,398)$. In this study, in stable patients without clinical worsening within 72 hours, the decision to prevent decompression was taken by formulating the ratio of infarct volume to cerebellar volume; GCS $\geq 9$ patients were also included in the preventive suboccipital decompression group (397).

Patients with a large cerebellar infarction should be kept under observation, preferably in the neurology intensive care unit, in the early period due to the risk of mass effect, hydrocephalus, and herniation. It should not be forgotten that patients with large cerebellar infarcts may worsen rapidly and should be followed with great care, especially in the first 5 days, for a chance of surgical intervention (282).

\subsection{Decompressive Craniectomy in Intracerebral Hemorrhages}

Although there are studies showing that decompressive craniectomy can be successfully applied in intracerebral hemorrhages, the data on this subject are based on case series or casecontrol studies (399-402).

Spontaneous intracerebral hemorrhage (ICH) is a devastating condition that accounts for 10$15 \%$ of all strokes. The mortality rate is high, and the rate is reported as $30 \%-50 \%$ in the first 30 
days. Also, the majority of the survivors continue their lives with severe disabilities (403). Bleeding affects the brain both directly and indirectly. Its direct effect occurs with the destruction of brain tissue. It exerts its indirect effect by causing an increase in intracranial pressure. Surgical evacuation of the hematoma has been investigated in patients with intracerebral hemorrhage unresponsive to medical therapy, with disappointing results (404). Two recent studies, the International Surgical Trial in Intracerebral Hemorrhage (STICH and STICH II), also failed to show a benefit of hematoma evacuation. The STICH I study compared conservative treatment and surgical evacuation of the hematoma at an early stage in patients with spontaneous supratentorial ICH and found no difference in the 6-month neurological outcome. However, it was found that patients with hematoma closer than 10 $\mathrm{mm}$ to the cortex surface (superficial lobar hematoma) partially benefited from early surgery. Similarly, no benefit was identified in the STICH II study $(405,406)$. Therefore, there was a need for a new concept. This new concept was decompressive craniectomy.

However, there are some questions that complicate the decision to perform a decompressive craniectomy: in which patients is it appropriate, what is the correct timing, and what will be the result when performed?

Decompressive craniectomy in supratentorial intracerebral hemorrhages: According to the AHA/ASA and ESO guidelines for the management of spontaneous intracerebral hemorrhage (407), decompressive surgery with or without hematoma evacuation reduces mortality in patients with supratentorial large hematoma with significant midline shift, in a coma, and with increased intracranial pressure resistant to medical therapy (Class IIb, level of evidence C). A systematic review also indicated that performing decompressive craniectomy with hematoma evacuation gives better results (408).

Decompressive craniectomy in posterior fossa hemorrhages, cerebellar hematoma surgery: Evacuation of cerebellar hematoma causing mass effect is a standard treatment. Decompressive craniectomy is performed as part of surgery to make room for potential post-operative swelling (409). AHA/ASA guidelines (Class I, Level of Evidence B) recommend direct surgical evacuation for cerebellar hemorrhages greater than $3 \mathrm{~cm}$
(410). The location of the hematoma may be more effective than the size; Proximity to the brain stem and increased intracranial pressure facilitate the decision for surgery. Although there are studies indicating that suboccipital decompressive craniectomy reduces mortality in patients with acute hydrocephalus, brainstem compression, or posterior fossa hemorrhage with worsening neurological status, high-quality multi-centered randomized studies are also needed(409). Decompressive craniectomy may also be lifesaving in cases of cerebral venous thrombosis with malignant venous infarcts and hematomas (411).

"The Swiss Trial of Decompressive Craniectomy Versus Best Medical Treatment of Spontaneous Supratentorial Intracerebral Hemorrhage" (SWITCH) is an ongoing prospective, randomized study investigating whether decompressive craniectomy will be a safe method in deep ICH and its effect on mortality and morbidity. This study compares the patient group receiving decompressive craniectomy and optimal medical treatment together with the group receiving optimal medical treatment alone. This study, which started in October 2014, is based in Europe, the last patient recruitment is planned to be completed in September 2023 and the first results are expected to be announced in March 2024 (ClinicalTrials.gov Identifier: NCT022589).

In conclusion, decompressive craniectomy with hematoma evacuation provides life-saving results in patients with a large supratentorial hemorrhage with a midline shift of more than 5 $\mathrm{mm}$ and refractory increase in ICP that does not respond to medical treatment, and in those with cerebellar hemorrhage greater than $3 \mathrm{~cm}$, which compresses the brainstem structures, has signs of herniation, and develops acute hydrocephalus (Image 20-21).

\subsection{Afterword}

Acute stroke cases constitute an important and large part of the inpatient profile in Neurology Intensive Care Units. Ischemic stroke constitutes $80-85 \%$ of the cases, and intracerebral hemorrhages should be followed carefully in terms of ICP due to the high mortality risk. $40 \%$ of patients with large cerebral infarctions (anterior system total vascular occlusions) worsen in the first week, and half of those who get worse die in the first month. In these cases, the timely detection of cerebral edema and IICP and the correct 


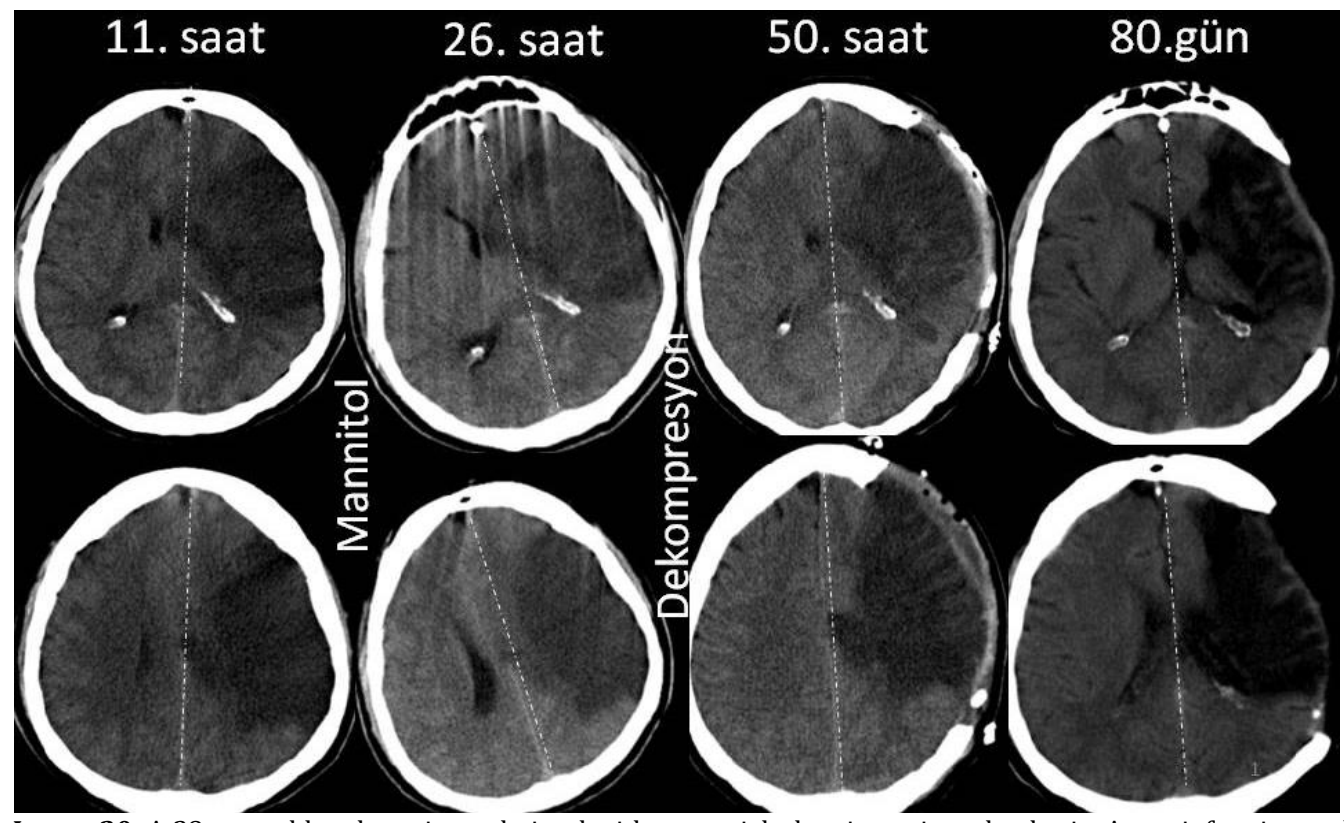

Image 20. A 28-year-old male patient admitted with acute right hemiparesis and aphasia. Acute infarction and mild shift are seen on the 11th hour CT. When the clinical worsening continues after the start of mannitol, the CT taken at the 26th hour shows that edema and shifting effect increase, and decompressive hemicraniectomy is performed. In the CT taken after decompression at the 50th hour, the midline shift is not completely resolved. Control CT taken on the 80th day shows that the infarct area has completely atrophied, and cranial revision surgery is performed. At the end of the first year, the patient is able to walk and speak (dashed line - . indicates midline).

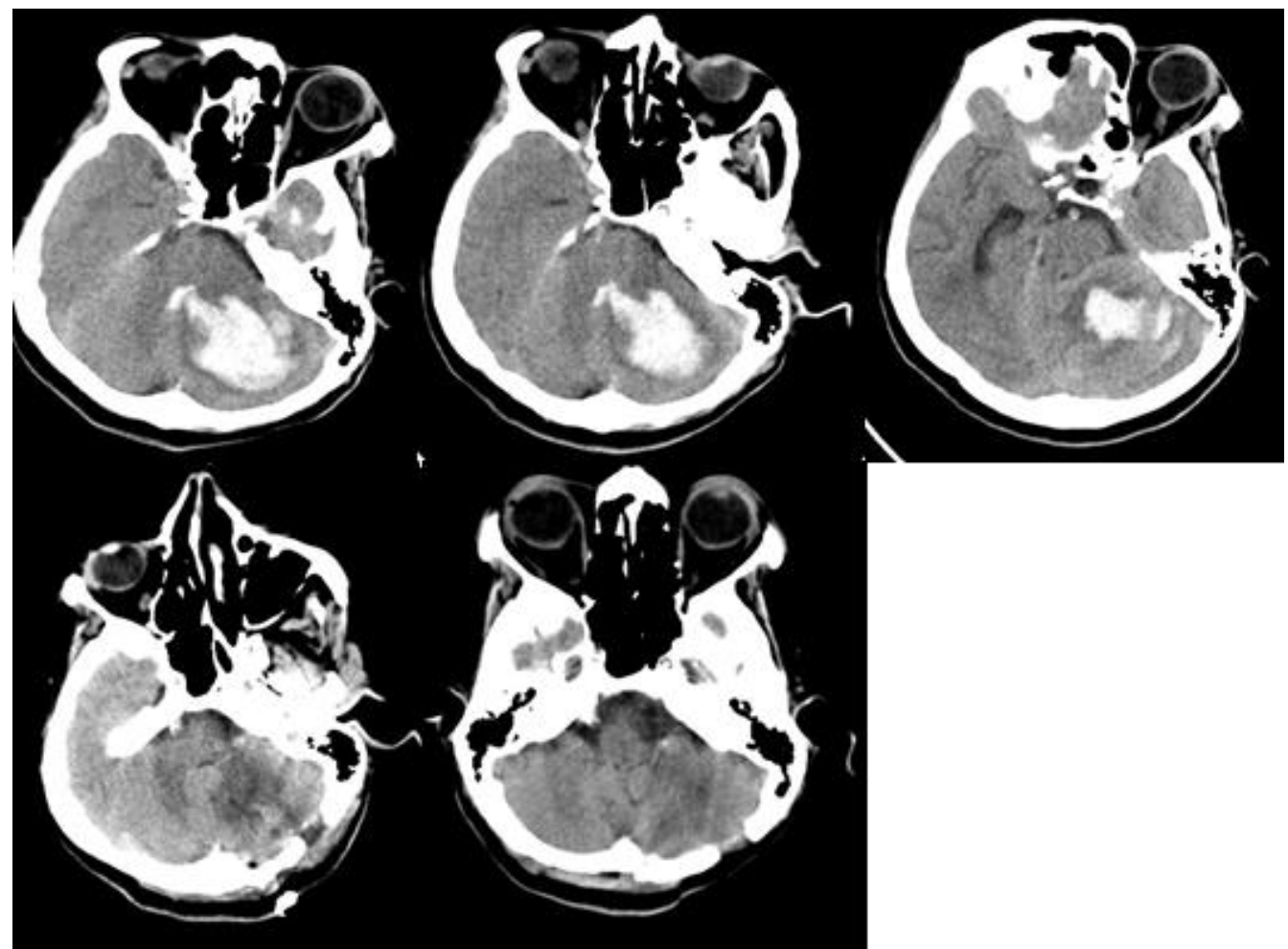

Image 21: Release of pressure on brainstem structures and 4th ventricle after suboccipital decompression in serial brain CT scans in a patient with left cerebellar hematoma. 
approach are the most important factors determining the prognosis. Osmotherapy has been shown to reduce intracranial pressure, despite the limited evidence-based applications due to the insufficiency of placebo-controlled randomized double-blind studies showing the efficacy of osmotherapy in cerebral edema treatment. In the treatment of antiedema with mannitol and hypertonic fluids, close follow-up and laboratory follow-up of patients are critical, especially considering the side effects and complications. When focal neurological findings suggestive of unconsciousness, neurological progression, and herniation are detected in the clinic, further investigations should be performed, and medical and surgical interventions should be performed on time. Midline shift and brainstem compression due to brain edema in large cerebellar infarcts in the posterior system are causes of mortality. Cases can progress rapidly, consciousness monitoring and clinical follow-up are crucial. Treatment of brain edema in intracerebral hemorrhages is controversial, and they should be followed up medically in terms of ICP and referred to surgical treatment in suitable cases.

In case of increased intracranial pressure, the first thing to do is to elevate the head of the bed by $30^{\circ}$, which will increse venous return. In addition, factors that increase intracranial pressure, such as hypoxia, hypercarbia, hyperthermia, hyperglycemia, and antihypertensive drugs (especially those that cause cerebral vasodilation) should be avoided. Osmotherapy and, if necessary, decompression surgery should be applied to selected patients.

We hope that this review, which explains the physiopathology of cerebral edema developing in ischemic stroke and intracerebral hemorrhage, the clinical and radiological appearance, and the laboratory methods to be used for the detection and follow-up of rICP, and its treatment, will be helpful.

\section{REFERENCES}

1. Hewitt A, Ellory CJS. Brain oedema, intracranial pressure and cerebral blood flow. Surgery (Oxford) 2012; 30(3): 102-106.

2. Shardlow E, Jackson AJA, Medicine IC. Cerebral blood flow and intracranial pressure. Anaesthesia and Intensive Care Medicine 2008; 9(5): 222-225.

3. Michinaga S, Koyama YJIjoms. Pathogenesis of brain edema and investigation into anti-edema drugs. Int J Mol Sci 2015; 16(5): 9949-9975.
4. Thomalla G, Hartmann F, Juettler E, et al. Prediction of malignant middle cerebral artery infarction by magnetic resonance imaging within 6 hours of symptom onset: A prospective multicenter observational study. Ann Neurol 2010; 68(4): 435-445.

5. Hacke W, Schwab S, Horn M, et al. 'Malignant'middle cerebral artery territory infarction: Clinical course and prognostic signs. Arch Neurol 1996; 53(4): 309-315.

6. Kase C, Norrving B, Levine S, et al. Cerebellar infarction. Clinical and anatomic observations in 66 cases. Stroke 1993; 24(1): 76-83.

7. Koh MG, Phan TG, Atkinson JL, et al. Neuroimaging in deteriorating patients with cerebellar infarcts and mass effect. Stroke 2000; 31(9): 2062-2067.

8. Xi G, Hua Y, Bhasin RR, et al. Mechanisms of edema formation after intracerebral hemorrhage: Effects of extravasated red blood cells on blood flow and blood-brain barrier integrity. Stroke 2001; 32(12): 2932-2938.

9. Xi G, Keep RF, Hoff JTJJon. Erythrocytes and delayed brain edema formation following intracerebral hemorrhage in rats. Journal of Neurosurgery 1998; 89(6): 991-996.

10. Rosand J, Schwamm LHJJoICM. Management of brain edema complicating stroke. Journal of Intensive Care Medicine 2001; 16(3): 128-141.

11. Wijdicks EF, Sheth KN, Carter BS, et al. Recommendations for the management of cerebral and cerebellar infarction with swelling: A statement for healthcare professionals from the american heart association/american stroke association. Stroke, 2014; 45(4): 1222-1238.

12. Brogan ME, Manno EMJCtoin. Treatment of malignant brain edema and increased intracranial pressure after stroke. Curr Treat Options Neurol 2015; 17(1): 327.

13. Wu S, Yuan R, Wang Y, et al. Early prediction of malignant brain edema after ischemic stroke: A systematic review and meta-analysis. Stroke 2018; 49(12): 2918-2927.

14. Siegel J, Pizzi MA, Peel JB, et al. Update on neurocritical care of stroke. Curr Cardiol Rep 2017; 19(8): 1-11.

15. Neugebauer H, Witsch J, Zweckberger K, et al. Spaceoccupying cerebellar infarction: Complications, treatment, and outcome. Neurosurgical Focus 2013; 34(5): E8.

16. Edlow JA, Newman-Toker DE, Savitz SI. Diagnosis and initial management of cerebellar infarction. The Lancet Neurology 2008; 7(10): 951-964.

17. Poon MTC, Fonville AF, Salman RA-SJJoN, Neurosurgery, et al. Long-term prognosis after intracerebral haemorrhage: Systematic review and meta-analysis. J Neurol Neurosurg Psychiatry 2014; 85(6): 660-667.

18. Butcher KS, Baird T, MacGregor L, et al. Perihematomal edema in primary intracerebral hemorrhage is plasma derived. Stroke 2004; 35(8): 1879-1885.

19. Tsai Y-H, Hsu L-M, Weng H-H, et al. Voxel-based analysis of apparent diffusion coefficient in perihaematomal oedema: Associated factors and outcome predictive value for intracerebral haemorrhage. BMJ Open 2011; 1(1).

20. Appelboom G, Bruce SS, Hickman ZL, et al. Volumedependent effect of perihaematomal oedema on outcome for spontaneous intracerebral haemorrhages. J Neurol Neurosurg Psychiatry 2013; 84(5): 488-493.

21. Arima H, Wang J, Huang $Y$, et al. Significance of perihematomal edema in acute intracerebral hemorrhage: The interact trial. Neurology 2009; 73(23): 1963-1968.

22. Anderson CS, Huang Y, Wang JG, et al. Intensive blood pressure reduction in acute cerebral haemorrhage trial (interact): A randomised pilot trial. The Lancet Neurology 2008; 7(5): 391-399. 
23. Anderson CS, Heeley E, Huang Y, et al. Rapid bloodpressure lowering in patients with acute intracerebral hemorrhage. N Engl J Med 2013; 368: 2355-2365.

24. Yang J, Arima H, Wu G, et al. Prognostic significance of perihematomal edema in acute intracerebral hemorrhage: Pooled analysis from the intensive blood pressure reduction in acute cerebral hemorrhage trial studies. Stroke 2015; 46(4): 1009-1013.

25. Yu Z, Ma L, Zheng J, et al. Prognostic role of perihematomal edema in intracerebral hemorrhage: A systematic review. Turk Neurosurg 2018; 28(4): 511-522.

26. Murthy SB, Urday S, Beslow LA, et al. Rate of perihaematomal oedema expansion is associated with poor clinical outcomes in intracerebral haemorrhage. J Neurol Neurosurg Psychiatry 2016; 87(11): 1169-1173.

27. Wu TY, Sharma G, Strbian D, et al. Natural history of perihematomal edema and impact on outcome after intracerebral hemorrhage. Stroke 2017; 48(4): 873-879.

28. Selim M, Norton CJJonr. Perihematomal edema: Implications for intracerebral hemorrhage research and therapeutic advances. J Neuro Res 2020; 98(1): 212-218.

29. Battey TW, Karki M, Singhal AB, et al. Brain edema predicts outcome after nonlacunar ischemic stroke. Stroke 2014; 45(12): 3643-3648.

30. Yao Y, Zhang Y, Liao X, et al. Potential therapies for cerebral edema after ischemic stroke: A mini review. Front Aging Neurosci 2020; 12: 618819.

31. Monro A. Observations on the structure and functions of the nervous system illustrated with tables. London; 1835.

32. Kellie GJTM-CSoE. An account of the appearances observed in the dissection of two of three individuals presumed to have perished in the storm of the $3 \mathrm{~d}$, and whose bodies were discovered in the vicinity of leith on the morning of the 4 th, november 1821; with some reflections on the pathology of the brain: Part i. Trans Med Chir Soc Edinb 1824; 1: 84-122.

33. Fink MEJCLLiN. Osmotherapy for intracranial hypertension: Mannitol versus hypertonic saline. Critical Care Neurology 2012; 18(3): 640-654.

34. Wilson MH. Monro-kellie 2.0: The dynamic vascular and venous pathophysiological components of intracranial pressure. Journal of Cerebral Blood Flow \& Metabolism 2016; 36(8): 1338-1350.

35. Bradley WG, Safar FG, Furtado C, et al. Increased intracranial volume: A clue to the etiology of idiopathic normal-pressure hydrocephalus? AJNR Am J Neuroradiol 2004; 25(9): 1479-1484.

36. Ropper AH. Hyperosmolar therapy for raised intracranial pressure. N Engl J Med 2012; 367(8): 746-752.

37. Iadecola $\mathrm{C}$, Anrather J. The immunology of stroke: From mechanisms to translation. Nature Medicine 2011; 17(7): 796-808.

38. Macrez R, Ali C, Toutirais O, et al. Stroke and the immune system: From pathophysiology to new therapeutic strategies. Lancet Neurol 2011; 10(5): 471-480.

39. Bernardo-Castro S, Sousa JA, Brás A, et al. Pathophysiology of blood-brain barrier permeability throughout the different stages of ischemic stroke and its implication on hemorrhagic transformation and recovery. Front Neurol 2020; 11: 594672 .

40. Simard JM, Kent TA, Chen M, et al. Brain oedema in focal ischaemia: Molecular pathophysiology and theoretical implications. The Lancet Neurology 2007; 6(3): 258-268.

41. Platten M, Wick W. Chapter 5 - blood-brain barrier and brain edema. In: Aminoff MJ, Boller F, Swaab DF, editors.
Handbook of clinical neurology. 104. : Elsevier; 2012; 53 62.

42. Badaut J, Ashwal S, Obenaus A. Aquaporins in cerebrovascular disease: A target for treatment of brain edema. Cerebrovascular Diseases 2011; 31(6): 521-531.

43. Chamorro Á, Meisel A, Planas AM, et al. The immunology of acute stroke. Nat Rev Neurol 2012; 8(7): 401-410.

44. Stokum JA, Kwon MS, Woo SK, et al. Sur1-trpm4 and aqp4 form a heteromultimeric complex that amplifies ion/water osmotic coupling and drives astrocyte swelling. Glia 2018; 66(1): 108-125.

45. Nag S, Manias JL, Stewart DJ. Pathology and new players in the pathogenesis of brain edema. Acta Neuropathol 2009; 118(2): 197-217.

46. Watts ME, Pocock R, Claudianos C. Brain energy and oxygen metabolism: Emerging role in normal function and disease. Front. Mol. Neurosci 2018; 11(216).

47. Prakash R, Carmichael ST. Blood-brain barrier breakdown and neovascularization processes after stroke and traumatic brain injury. Curr Opin Neurol 2015; 28(6): 556564.

48. Sweeney MD, Zhao Z, Montagne A, et al. Blood-brain barrier: From physiology to disease and back. Physiol Rev 2019; 99(1): 21-78.

49. Abbott NJ, Patabendige AA, Dolman DE, et al. Structure and function of the blood-brain barrier. Neurobiol Dis 2010; 37(1): 13-25.

50. Attwell D, Buchan AM, Charpak S, et al. Glial and neuronal control of brain blood flow. Nature 2010; 468(7321): 232243.

51. Alarcon-Martinez L, Yemisci M, Dalkara T. Pericyte morphology and function. Histology and Histopathology 2021; 36(6): 505-514.

52. Zlokovic BV. Neurovascular pathways to neurodegeneration in alzheimer's disease and other disorders. Nat Rev Neurosci 2011; 12(12): 723-738.

53. Iadecola $\mathrm{C}$. The neurovascular unit coming of age: A journey through neurovascular coupling in health and disease. Neuron 2017; 96(1): 17-42.

54. Armulik A, Genové G, Mäe M, et al. Pericytes regulate the blood-brain barrier. Nature 2010; 468(7323): 557-561.

55. Daneman R, Prat A. The blood-brain barrier. Cold Spring Harb Perspect Biol 2015; 7(1): a020412.

56. Alarcon-Martinez L, Yilmaz-Ozcan S, Yemisci $M$, et al. Capillary pericytes express $\alpha$-smooth muscle actin, which requires prevention of filamentous-actin depolymerization for detection. eLife 2018;7:e34861.

57. Stokum JA, Gerzanich V, Simard JM. Molecular pathophysiology of cerebral edema. J Cereb Blood Flow Metab 2016; 36(3): 513-538.

58. Steiner E, Enzmann GU, Lin S, et al. Loss of astrocyte polarization upon transient focal brain ischemia as a possible mechanism to counteract early edema formation. Glia 2012; 60(11): 1646-1659.

59. Yemisci M, Gursoy Ozdemir Y, Vural A, et al. Pericyte contraction induced by oxidative-nitrative stress impairs capillary reflow despite successful opening of an occluded cerebral artery. Nat Med 2009; 15(9): 1031-1037.

60. Dalkara T, Alarcon-Martinez L, Yemisci M. Pericytes in ischemic stroke. Adv Exp Med Biol 2019; 1147: 189-213.

61. Ehrlich PR. Das sauerstoff-bediirfniss des organismus. A Hirschwald, Berlin 1885.

62. Oldendorf WH, Cornford ME, Brown WJ. The large apparent work capability of the blood-brain barrier: A study of the mitochondrial content of capillary endothelial cells in brain 
Güngör et al.

and other tissues of the rat. Ann Neurol 1977; 1(5): 409417.

63. Gürsoy Özdemir Y, Tas YC. Chapter 1 - anatomy and physiology of the blood-brain barrier. In: Gürsoy-Özdemir Y, Bozdağ-Pehlivan S, Sekerdag E, editors. Nanotechnology methods for neurological diseases and brain tumors: Academic Press; 2017; 3-13.

64. Pappenheimer JR, Renkin EM, Borrero LM. Filtration, diffusion and molecular sieving through peripheral capillary membranes; a contribution to the pore theory of capillary permeability. Am J Physiol 1951; 167(1): 13-46.

65. Siegenthaler JA, Sohet F, Daneman R. 'Sealing off the cns': Cellular and molecular regulation of blood-brain barriergenesis. Curr Opin Neurobiol 2013; 23(6): 10571064.

66. Liu WY, Wang ZB, Zhang LC, et al. Tight junction in bloodbrain barrier: An overview of structure, regulation, and regulator substances. CNS Neurosci Ther 2012; 18(8): 609615.

67. Nitta T, Hata M, Gotoh S, et al. Size-selective loosening of the blood-brain barrier in claudin-5-deficient mice. J Cell Biol 2003; 161(3): 653-660.

68. Gürsoy-Ozdemir Y, Bolay $H$, Saribaș 0 , et al. Role of endothelial nitric oxide generation and peroxynitrite formation in reperfusion injury after focal cerebral ischemia. Stroke 2000; 31(8): 1974-1980; discussion 1981.

69. Krizbai IA, Deli MA. Signalling pathways regulating the tight junction permeability in the blood-brain barrier. Cell Mol Biol (Noisy-le-grand) 2003; 49(1): 23-31.

70. Jiang X, Andjelkovic AV, Zhu L, et al. Blood-brain barrier dysfunction and recovery after ischemic stroke. Prog Neurobiol 2018; 163-164: 144-171.

71. Song L, Ge S, Pachter JS. Caveolin-1 regulates expression of junction-associated proteins in brain microvascular endothelial cells. Blood 2007; 109(4): 1515-1523.

72. Gürsoy-Ozdemir Y, Can A, Dalkara T. Reperfusion-induced oxidative/nitrative injury to neurovascular unit after focal cerebral ischemia. Stroke 2004; 35(6): 1449-1453.

73. Gibson CL, Srivastava K, Sprigg N, et al. Inhibition of rhokinase protects cerebral barrier from ischaemia-evoked injury through modulations of endothelial cell oxidative stress and tight junctions. J Neurochem 2014; 129(5): 816826.

74. Haas M, Forbush B, 3rd. The na-k-cl cotransporter of secretory epithelia. Annu Rev Physiol 2000; 62: 515-534.

75. Russell JM. Sodium-potassium-chloride cotransport. Physiol Rev 2000; 80(1): 211-276.

76. Xu JC, Lytle C, Zhu TT, et al. Molecular cloning and functional expression of the bumetanide-sensitive na-k-cl cotransporter. Proc Natl Acad Sci USA 1994; 91(6): 22012205.

77. Delpire E, Mount DB. Human and murine phenotypes associated with defects in cation-chloride cotransport. Annu Rev Physiol 2002; 64: 803-843.

78. Sun D, Lytle C, O'Donnell ME. Astroglial cell-induced expression of na-k-cl cotransporter in brain microvascular endothelial cells. Am J Physiol 1995; 269(6 Pt 1): C15061512.

79. Wu Q, Delpire E, Hebert SC, et al. Functional demonstration of na+-k+-2cl- cotransporter activity in isolated, polarized choroid plexus cells. Am J Physiol 1998; 275(6): C15651572.

80. Yan Y, Dempsey RJ, Sun D. Na+-k+-cl- cotransporter in rat focal cerebral ischemia. J Cereb Blood Flow Metab 2001; 21(6): 711-721.
81. Wang H, Yan Y, Kintner DB, et al. Gaba-mediated trophic effect on oligodendrocytes requires na-k-2cl cotransport activity. J Neurophysiol 2003; 90(2): 1257-1265.

82. Chen H, Sun D. The role of na-k-cl co-transporter in cerebral ischemia. Neurol Res 2005; 27(3): 280-286.

83. Beck J, Lenart B, Kintner DB, et al. Na-k-cl cotransporter contributes to glutamate-mediated excitotoxicity. J Neurosci 2003; 23(12): 5061-5068.

84. Markadieu N, Delpire E. Physiology and pathophysiology of slc12a1/2 transporters. Pflugers Arch 2014; 466(1): 91105.

85. Su G, Kintner DB, Flagella M, et al. Astrocytes from na(+)$\mathrm{k}(+)-\mathrm{cl}(-)$ cotransporter-null mice exhibit absence of swelling and decrease in eaa release. Am J Physiol Cell Physiol 2002; 282(5): C1147-1160.

86. Su G, Kintner DB, Sun D. Contribution of na(+)-k(+)-cl(-) cotransporter to high-[k(+)](0)- induced swelling and eaa release in astrocytes. Am J Physiol Cell Physiol 2002; 282(5): C1136-1146.

87. Schomberg SL, Su G, Haworth RA, et al. Stimulation of na-k$2 \mathrm{cl}$ cotransporter in neurons by activation of non-nmda ionotropic receptor and group-i mglurs. J Neurophysiol 2001; 85(6): 2563-2575.

88. Leppanen L, Stys PK. Ion transport and membrane potential in cns myelinated axons. Ii. Effects of metabolic inhibition. J Neurophysiol 1997; 78(4): 2095-2107.

89. Stys PK, Waxman SG, Ransom BR. Ionic mechanisms of anoxic injury in mammalian cns white matter: Role of na+ channels and na(+)-ca2+ exchanger. J Neurosci 1992; 12(2): 430-439.

90. O'Donnell ME, Tran L, Lam TI, et al. Bumetanide inhibition of the blood-brain barrier na-k-cl cotransporter reduces edema formation in the rat middle cerebral artery occlusion model of stroke. J Cereb Blood Flow Metab 2004; 24(9): 1046-1056.

91. Huang $H$, Bhuiyan MIH, Jiang $T$, et al. A novel na(+)-k(+)cl(-) cotransporter 1 inhibitor sts66* reduces brain damage in mice after ischemic stroke. Stroke 2019; 50(4): 10211025.

92. Kahle KT, Simard JM, Staley KJ, et al. Molecular mechanisms of ischemic cerebral edema: Role of electroneutral ion transport. Physiology (Bethesda) 2009; 24: 257-265.

93. Rothman SM. The neurotoxicity of excitatory amino acids is produced by passive chloride influx. J Neurosci 1985; 5(6): 1483-1489.

94. Choi DW, Maulucci-Gedde M, Kriegstein AR. Glutamate neurotoxicity in cortical cell culture. J Neurosci 1987; 7(2): 357-368.

95. Aittoniemi J, Fotinou C, Craig TJ, et al. Review. Sur1: A unique atp-binding cassette protein that functions as an ion channel regulator. Philos Trans R Soc Lond B Biol Sci 2009; 364(1514): 257-267.

96. Simard JM, Woo SK, Gerzanich V. Transient receptor potential melastatin 4 and cell death. Pflugers Arch 2012; 464(6): 573-582.

97. Simard JM, Woo SK, Schwartzbauer GT, et al. Sulfonylurea receptor 1 in central nervous system injury: A focused review. J Cereb Blood Flow Metab 2012; 32(9): 1699-1717.

98. Vennekens R, Nilius B. Insights into trpm4 function, regulation and physiological role. Handb Exp Pharmacol 2007; (179): 269-285.

99. Mehta RI, Tosun C, Ivanova S, et al. Sur1-trpm4 cation channel expression in human cerebral infarcts. J Neuropathol Exp Neurol 2015; 74(8): 835-849.

100. Chen M, Simard JM. Cell swelling and a nonselective

Turkish Journal of Cerebrovascular Diseases 2021; 27(2): 65-132 
cation channel regulated by internal ca2+ and atp in native reactive astrocytes from adult rat brain. J Neurosci 2001; 21(17): 6512-6521.

101. Jha RM, Puccio AM, Chou SH, et al. Sulfonylurea receptor1: A novel biomarker for cerebral edema in severe traumatic brain injury. Crit Care Med 2017; 45(3): e255e264.

102. Nilius B, Owsianik G. The transient receptor potential family of ion channels. Genome Biol 2011; 12(3): 218.

103. Woo SK, Kwon MS, Ivanov A, et al. The sulfonylurea receptor 1 (sur1)-transient receptor potential melastatin 4 (trpm4) channel. J Biol Chem 2013; 288(5): 3655-3667.

104. Mathar I, Jacobs G, Kecskes M, et al. Trpm4. Handb Exp Pharmacol 2014; 222: 461-487.

105. Gerzanich V, Woo SK, Vennekens R, et al. De novo expression of trpm 4 initiates secondary hemorrhage in spinal cord injury. Nat Med 2009; 15(2): 185-191.

106. Tosun C, Kurland DB, Mehta R, et al. Inhibition of the sur1-trpm4 channel reduces neuroinflammation and cognitive impairment in subarachnoid hemorrhage. Stroke 2013; 44(12): 3522-3528.

107. Woo SK, Tsymbalyuk N, Tsymbalyuk 0, et al. Sur1-trpm4 channels, not $\mathrm{k}(\mathrm{atp})$, mediate brain swelling following cerebral ischemia. Neurosci Lett 2020; 718: 134729.

108. Loh KP, Ng G, Yu CY, et al. Trpm4 inhibition promotes angiogenesis after ischemic stroke. Pflugers Arch 2014; 466(3): 563-576.

109. Simard JM, Chen M, Tarasov KV, et al. Newly expressed sur1-regulated nc(ca-atp) channel mediates cerebral edema after ischemic stroke. Nat Med 2006; 12(4): 433440.

110. Rajasekaran SA, Beyenbach KW, Rajasekaran AK. Interactions of tight junctions with membrane channels and transporters. Biochim Biophys Acta 2008; 1778(3): 757-769.

111. Day RE, Kitchen P, Owen DS, et al. Human aquaporins: Regulators of transcellular water flow. Biochim Biophys Acta 2014; 1840(5): 1492-1506.

112. Gonen T, Walz T. The structure of aquaporins. Q Rev Biophys 2006; 39(4): 361-396.

113. Murata K, Mitsuoka $\mathrm{K}$, Hirai $\mathrm{T}$, et al. Structural determinants of water permeation through aquaporin-1. Nature 2000; 407(6804): 599-605.

114. Jung JS, Preston GM, Smith BL, et al. Molecular structure of the water channel through aquaporin chip. The hourglass model. J Biol Chem 1994; 269(20): 1464814654.

115. Chu H, Huang $\mathrm{C}$, Ding $\mathrm{H}$, et al. Aquaporin-4 and cerebrovascular diseases. Int J Mol Sci 2016; 17(8).

116. Papadopoulos MC, Verkman AS. Aquaporin-4 and brain edema. Pediatr Nephrol 2007; 22(6): 778-784.

117. Jullienne A, Fukuda AM, Ichkova A, et al. Modulating the water channel aqp4 alters mirna expression, astrocyte connectivity and water diffusion in the rodent brain. Sci Rep 2018; 8(1): 4186.

118. Ribeiro Mde C, Hirt L, Bogousslavsky J, et al. Time course of aquaporin expression after transient focal cerebral ischemia in mice. J Neurosci Res 2006; 83(7): 1231-1240.

119. Verkman AS, Anderson MO, Papadopoulos MC. Aquaporins: Important but elusive drug targets. Nat Rev Drug Discov 2014; 13(4): 259-277.

120. Amiry-Moghaddam M, Williamson A, Palomba M, et al. Delayed $\mathrm{k}+$ clearance associated with aquaporin-4 mislocalization: Phenotypic defects in brains of alpha- syntrophin-null mice. Proc Natl Acad Sci U S A 2003; 100(23): 13615-13620.

121. Zeng XN, Xie LL, Liang R, et al. Aqp4 knockout aggravates ischemia/reperfusion injury in mice. CNS Neurosci Ther 2012; 18(5): 388-394.

122. Yang B, Verkman AS. Water and glycerol permeabilities of aquaporins 1-5 and mip determined quantitatively by expression of epitope-tagged constructs in xenopus oocytes. J Biol Chem 1997; 272(26): 16140-16146.

123. Akdemir G, Ratelade J, Asavapanumas N, et al. Neuroprotective effect of aquaporin-4 deficiency in a mouse model of severe global cerebral ischemia produced by transient 4 -vessel occlusion. Neurosci Lett 2014; 574: 70-75.

124. Hirt L, Fukuda AM, Ambadipudi K, et al. Improved longterm outcome after transient cerebral ischemia in aquaporin-4 knockout mice. J Cereb Blood Flow Metab 2017; 37(1): 277-290.

125. Stokum JA, Mehta RI, Ivanova S, et al. Heterogeneity of aquaporin-4 localization and expression after focal cerebral ischemia underlies differences in white versus grey matter swelling. Acta Neuropathol Commun 2015; 3: 61.

126. Nakahama K, Nagano M, Fujioka A, et al. Effect of tpa on aquaporin 4 mrna expression in cultured rat astrocytes. Glia 1999; 25(3): 240-246.

127. Hirt L, Ternon B, Price M, et al. Protective role of early aquaporin 4 induction against postischemic edema formation. J Cereb Blood Flow Metab 2009; 29(2): 423 433.

128. Cheripelli BK, Huang $X$, MacIsaac R, et al. Interaction of recanalization, intracerebral hemorrhage, and cerebral edema after intravenous thrombolysis. Stroke 2016; 47(7): 1761-1767.

129. Simard JM, Kent TA, Chen M, et al. Brain oedema in focal ischaemia: Molecular pathophysiology and theoretical implications. Lancet Neurol 2007; 6(3): 258-268.

130. Bai J, Lyden PD. Revisiting cerebral postischemic reperfusion injury: New insights in understanding reperfusion failure, hemorrhage, and edema. International Journal of Stroke 2015; 10(2): 143-152.

131. Pun PB, Lu J, Moochhala S. Involvement of ros in bbb dysfunction. Free radical research 2009; 43(4): 348-364.

132. Jin G, Arai K, Murata $Y$, et al. Protecting against cerebrovascular injury: Contributions of 12/15lipoxygenase to edema formation after transient focal ischemia. Stroke 2008; 39(9): 2538-2543.

133. Heo JH, Han SW, Lee SK. Free radicals as triggers of brain edema formation after stroke. Free Radical Biology and Medicine 2005; 39(1): 51-70.

134. Schaller B, Graf R. Cerebral ischemia and reperfusion: The pathophysiologic concept as a basis for clinical therapy. Journal of Cerebral Blood Flow \& Metabolism 2004; 24(4): 351-371.

135. Kimberly WT, Dutra BG, Boers AM, et al. Association of reperfusion with brain edema in patients with acute ischemic stroke: A secondary analysis of the mr clean trial. JAMA neurology 2018; 75(4): 453-461.

136. Goyal M, Demchuk AM, Menon BK, et al. Randomized assessment of rapid endovascular treatment of ischemic stroke. New England Journal of Medicine 2015; 372(11): 1019-1030.

137. Cheripelli BK, Huang $X$, MacIsaac R, et al. Interaction of recanalization, intracerebral hemorrhage, and cerebral 
edema after intravenous thrombolysis. Stroke 2016; 47(7): 1761-1767.

138. Goyal N, Tsivgoulis G, Pandhi A, et al. Blood pressure levels post mechanical thrombectomy and outcomes in large vessel occlusion strokes. Neurology 2017; 89(6): 540-547.

139. Ziai WC, Carhuapoma JR. Intracerebral hemorrhage. Continuum (Minneap Minn) 2018; 24(6): 1603-1622.

140. Ironside N, Chen CJ, Ding D, et al. Perihematomal edema after spontaneous intracerebral hemorrhage. Stroke 2019; 50(6): 1626-1633.

141. Xie S, Qin Z, Yin X. Classification mechanism and clinical analysis of perihematomal edema in intracerebral hemorrhage. Brain Hemorrhages 2020; 1(3): 141-145.

142. Selim M, Norton C. Perihematomal edema: Implications for intracerebral hemorrhage research and therapeutic advances. J Neurosci Res 2020; 98(1): 212-218.

143. Murthy SB, Moradiya Y, Dawson J, et al. Perihematomal edema and functional outcomes in intracerebral hemorrhage: Influence of hematoma volume and location. Stroke 2015; 46(11): 3088-3092.

144. Levine JM, Snider R, Finkelstein D, et al. Early edema in warfarin-related intracerebral hemorrhage. Neurocrit Care 2007; 7(1): 58-63.

145. Balci K, Asil T, Tunçbilek N, Celik Y, Utku U. Volume of perihematomal edema in diabetic patients. Journal of Turkish Cerebrovascular Diseases 2006; 12(3): 73-76.

146. Arima H, Wang JG, Huang Y, et al. Significance of perihematomal edema in acute intracerebral hemorrhage: The interact trial. Neurology 2009; 73(23): 1963-1968.

147. Qureshi AI, Palesch YY, Martin R, et al. Effect of systolic blood pressure reduction on hematoma expansion, perihematomal edema, and 3-month outcome among patients with intracerebral hemorrhage: Results from the antihypertensive treatment of acute cerebral hemorrhage study. Arch Neurol 2010; 67(5): 570-576.

148. Naval NS, Abdelhak TA, Urrunaga N, et al. An association of prior statin use with decreased perihematomal edema. Neurocrit Care 2008; 8(1): 13-18.

149. Yu Z, Ma L, Zheng J, et al. Prognostic role of perihematomal edema in intracerebral hemorrhage: A systematic review. Turk Neurosurg 2017; 28(4): 511522.

150. Johnson PL, Eckard DA, Chason DP, et al. Imaging of acquired cerebral herniations. Neuroimaging Clin N Am 2002; 12(2): 217-228.

151. Mokri B. The monro-kellie hypothesis: Applications in csf volume depletion. Neurology 2001; 56(12): 1746-1748.

152. Ryoo JW, Na DG, Kim SS, et al. Malignant middle cerebral artery infarction in hyperacute ischemic stroke: Evaluation with multiphasic perfusion computed tomography maps. J Comput Assist Tomogr 2004; 28(1): 55-62.

153. Coburn MW, Rodriguez FJ. Cerebral herniations. . Appl Radiol 1998; 27(5): 10-16.

154. Riveros Gilardi B, Muñoz López JI, Hernández Villegas AC, et al. Types of cerebral herniation and their imaging features. Radiographics 2019; 39(6): 1598-1610.

155. Ross DA, Olsen WL, Ross AM, et al. Brain shift, level of consciousness, and restoration of consciousness in patients with acute intracranial hematoma. J Neurosurg 1989; 71(4): 498-502.

156. Ropper AH. Lateral displacement of the brain and level of consciousness in patients with an acute hemispheral mass. N Engl J Med 1986; 314(15): 953-958.

157. Zhang $\mathrm{CH}$, DeSouza RM, Kho JS, et al. Kernohan-woltman notch phenomenon: A review article. Br J Neurosurg 2017; 31(2): 159-166.

158. Cuneo RA, Caronna JJ, Pitts L, et al. Upward transtentorial herniation: Seven cases and a literature review. Arch Neurol 1979; 36(10): 618-623.

159. Laine FJ, Shedden AI, Dunn MM, et al. Acquired intracranial herniations: $\mathrm{Mr}$ imaging findings. AJR Am J Roentgenol 1995; 165(4): 967-973.

160. Mikulis DJ, Diaz 0, Egglin TK, et al. Variance of the position of the cerebellar tonsils with age: Preliminary report. Radiology 1992; 183(3): 725-728.

161. Ishikawa $M$, Kikuchi $H$, Fujisawa $I$, et al. Tonsillar herniation on magnetic resonance imaging. Neurosurgery 1988; 22(1 Pt 1): 77-81.

162. Wu S, Yuan R, Wang Y, et al. Early prediction of malignant brain edema after ischemic stroke: A systematic review and meta-analysis. Stroke 2018; 49(12): 2918-2927.

163. Albert AF, Kirkman MA. Clinical and radiological predictors of malignant middle cerebral artery infarction development and outcomes. Journal of Stroke and Cerebrovascular Diseases 2017; 26(11): 2671-2679.

164. Krieger DW, Demchuk AM, Kasner SE, et al. Early clinical and radiological predictors of fatal brain swelling in ischemic stroke. Stroke 1999; 30(2): 287-292.

165. Kasner SE, Demchuk AM, Berrouschot Jr, et al. Predictors of fatal brain edema in massive hemispheric ischemic stroke. Stroke 2001; 32(9): 2117-2123.

166. Barber PA, Demchuk AM, Zhang J, et al. Computed tomographic parameters predicting fatal outcome in large middle cerebral artery infarction. Cerebrovascular Diseases 2003; 16(3): 230-235.

167. Jo K, Bajgur SS, Kim H, et al. A simple prediction score system for malignant brain edema progression in large hemispheric infarction. PLoS One 2017; 12(2): e0171425.

168. Ong CJ, Gluckstein J, Laurido-Soto O, et al. Enhanced detection of edema in malignant anterior circulation stroke (edema) score: A risk prediction tool. Stroke 2017; 48(7): 1969-1972.

169. Shimoyama T, Kimura K, Uemura J, et al. The dash score: A simple score to assess risk for development of malignant middle cerebral artery infarction. Journal of the neurological sciences 2014; 338(1-2): 102-106.

170. Madhok DY, Vitt JR, Nguyen AT. Overview of neurovascular physiology. Curr Neurol Neurosci Rep 2018; 18(12): 99.

171. Heinsius T, Bogousslavsky J, Van Melle G. Large infarcts in the middle cerebral artery territory. Etiology and outcome patterns. Neurology 1998; 50(2): 341-350.

172. Minnerup J, Wersching H, Ringelstein EB, et al. Prediction of malignant middle cerebral artery infarction using computed tomography-based intracranial volume reserve measurements. Stroke 2011; 42(12): 3403-3409.

173. Tei H, Uchiyama $\mathrm{S}$, Ohara $\mathrm{K}$, et al. Deteriorating ischemic stroke in 4 clinical categories classified by the oxfordshire community stroke project. Stroke 2000; 31(9): 2049-2054.

174. Liebeskind DS, Jüttler E, Shapovalov Y, et al. Cerebral edema associated with large hemispheric infarction. Stroke 2019; 50(9): 2619-2625.

175. Kılıç Çoban E, Selçuk HH, Kaykı Y, ve ark. Tanıda yanılgıya yol açan yaygın serebral ödem bulgusu; psödosubaraknoid kanama. Türk Beyin Damar Hastalıkları 
Dergisi 2015; 21(3): 213-216.

176. Topcuoglu MA, Unal A, Arsava EM. Advances in transcranial doppler clinical applications. Expert Opin Med Diagn 2010; 4(4): 343-358.

177. Evensen KB, Eide PK. Measuring intracranial pressure by invasive, less invasive or non-invasive means: Limitations and avenues for improvement. Fluids Barriers CNS 2020; 17(1): 34.

178. Tavakoli S, Peitz G, Ares W, et al. Complications of invasive intracranial pressure monitoring devices in neurocritical care. Neurosurg Focus 2017; 43(5): E6.

179. Cardim D, Robba C, Bohdanowicz M, et al. Non-invasive monitoring of intracranial pressure using transcranial doppler ultrasonography: Is it possible? Neurocrit Care 2016; 25(3): 473-491.

180. Klingelhöfer J, Conrad B, Benecke R, et al. Evaluation of intracranial pressure from transcranial doppler studies in cerebral disease. J Neurol 1988; 235(3): 159-162.

181. Wang Y, Duan YY, Zhou HY, et al. Middle cerebral arterial flow changes on transcranial color and spectral doppler sonography in patients with increased intracranial pressure. J Ultrasound Med 2014; 33(12): 2131-2136.

182. O'Brien NF, Maa T, Reuter-Rice K. Noninvasive screening for intracranial hypertension in children with acute, severe traumatic brain injury. J Neurosurg Pediatr 2015; 16(4): 420-425.

183. Ünal A. Dora B. Beyin ölümü tanısında destekleyici bir test olarak transkranial doppler ultrasonografisi. Türk Beyin Damar Hastalıkları Dergisi 2012; 18(3): 49-58.

184. Gosling R, King D. The role of measurement in peripheral vascular surgery: Arterial assessment by doppler-shift ultrasound. Proc R Soc Med 1974;67(6):447-449.

185. Bellner J, Romner B, Reinstrup P, et al. Transcranial Doppler sonography pulsatility index (PI)reflects intracranial pressure (ICP). Surg Neurol 2004; 62(1): 4551.

186. Figaji AA, Zwane E, Fieggen AG, et al. Transcranial Doppler pulsatility index is not a reliable indicator of intracranial pressure in children with severe traumatic brain injury. 2009; 72(4): 389-394.

187. de Riva N, Budohoski KP, Smielewski P, et al. Transcranial doppler pulsatility index: What it is and what it isn't. Neurocrit Care 2012; 17(1): 58-66.

188. Behrens A, Lenfeldt N, Ambarki K, et al. Transcranial doppler pulsatility index: Not an accurate method to assess intracranial pressure. Neurosurgery 2010; 66(6): 1050-1057.

189. Wakerley BR, Kusuma Y, Yeo LL, et al. Usefulness of transcranial doppler-derived cerebral hemodynamic parameters in the noninvasive assessment of intracranial pressure. J Neuroimaging 2015; 25(1): 111-116.

190. Asil T, Uzunca I, Utku U, et al. Monitoring of increased intracranial pressure resulting from cerebral edema with transcranial doppler sonography in patients with middle cerebral artery infarction. J Ultrasound Med 2003; 22(10): 1049-1053.

191. Barrozo HG, De Guzman MA, Navarro J, et al. Asymmetric TCD findings in malignant mca infarction, resolution after decompressive hemicraniectomy: A case report. Case Rep Neurol 2020; 12(Suppl 1): 127-136.

192. Topçuoğlu M, Arsava E. Kafa içi basınç artışı nörosonolojisi. Türk Beyin Damar Hastalıkları Dergisi 2011; 17(3): 77-87.

193. Topçuoğlu MA, Arsava EM. Transkranial renkli doppler ultrasonografide "polis çakar lambası (flaşör) işareti":
Beyin ölümü doğrulaması için az tanınan bir bulgu. Türk Beyin Damar Hastalıkları Dergisi 2014; 20(1): 36-37.

194. Gerriets T, Stolz E, Modrau B, et al. Sonographic monitoring of midline shift in hemispheric infarctions. Neurology 1999; 52(1): 45-45.

195. Wang L, Feng L, Yao Y, et al. Optimal optic nerve sheath diameter threshold for the identification of elevated opening pressure on lumbar puncture in a chinese population. PLoS One 2015; 10(2): e0117939.

196. Robba C, Bacigaluppi S, Cardim D, et al. Non-invasive assessment of intracranial pressure. Acta Neurol Scand 2016; 134(1): 4-21.

197. Gökcen E, Caltekin İ, Savrun A, et al. Alterations in optic nerve sheath diameter according to cerebrovascular disease sub-groups. Am J Emerg Med 2017; 35(11): 1607-1611.

198. Dubourg J, Javouhey E, Geeraerts T, et al. Ultrasonography of optic nerve sheath diameter for detection of raised intracranial pressure: A systematic review and meta-analysis. Intensive Care Med 2011; 37(7): 1059-1068

199. Lochner P, Czosnyka M, Naldi A, et al. Optic nerve sheath diameter: Present and future perspectives for neurologists and critical care physicians. Neurol Sci 2019; 40(12): 2447-2457.

200. Fowlkes JB, Holland CK. Mechanical bioeffects from diagnostic ultrasound: Aium consensus statements. American institute of ultrasound in medicine. J Ultrasound Med 2000; 19(2): 69-72.

201. Helmke K, Hansen HC. Fundamentals of transorbital sonographic evaluation of optic nerve sheath expansion under intracranial hypertension. I. Experimental study. Pediatr Radiol 1996; 26(10): 701-705.

202. Yüzbaşığlu Y, Yüzbaşığlu S, Coşkun S, et al. Bedside measurement of the optic nerve sheath diameter with ultrasound in cerebrovascular disorders. Turk J Med Sci 2018; 48(1): 93-99.

203. Güzeldağ S, Yılmaz G, Tuna M, et al. Measuring the optic nerve sheath diameter with ultrasound in acute middle cerebral artery stroke patients. J Stroke Cerebrovasc Dis 2021; 30(2): 105523.

204. Naldi A, Pivetta E, Coppo L, et al. Ultrasonography monitoring of optic nerve sheath diameter and retinal vessels in patients with cerebral hemorrhage. J Neuroimaging 2019; 29(3): 394-399.

205. Skoloudík D, Herzig R, Fadrná T, et al. Distal enlargement of the optic nerve sheath in the hyperacute stage of intracerebral haemorrhage. Br J Ophthalmol 2011; 95(2): 217-221.

206. Canac N, Jalaleddini $\mathrm{K}$, Thorpe SG, et al. Review: Pathophysiology of intracranial hypertension and noninvasive intracranial pressure monitoring. Fluids Barriers CNS 2020; 17(1): 40.

207. Packiasabapathy S, Rangasamy V, Sadhasivam S. Pupillometry in perioperative medicine: A narrative review. Can J Anaesth 2021; 68(4): 566-578.

208. Taylor WR, Chen JW, Meltzer H, et al. Quantitative pupillometry, a new technology: Normative data and preliminary observations in patients with acute head injury. Technical note. J Neurosurg 2003; 98(1): 205-213.

209. Chen JW, Gombart ZJ, Rogers S, et al. Pupillary reactivity as an early indicator of increased intracranial pressure: The introduction of the neurological pupil index. Surg Neurol Int 2011; 2: 82.

210. Samraj RS, Nicolas L. Near ifrared spectroscopy (NIRS) 
Güngör et al.

derived tissue oxygenation in critical illness. Clin Invest Med 2015; 38(5): E285-295.

211. Lorusso R, Taccone FS, Belliato M, et al. Brain monitoring in adult and pediatric ecmo patients: The importance of early and late assessments. Minerva Anestesiol 2017; 83(10): 1061-1074.

212. Weerakkody RA, Czosnyka M, Zweifel C, et al. Near infrared spectroscopy as possible non-invasive monitor of slow vasogenic icp waves. Acta Neurochir Suppl 2012; 114: 181-185.

213. Samuel M, Burge DM, Marchbanks RJ. Quantitative assessment of intracranial pressure by the tympanic membrane displacement audiometric technique in children with shunted hydrocephalus. Eur J Pediatr Surg 1998; 8(4): 200-207.

214. Shimbles S, Dodd C, Banister K, et al. Clinical comparison of tympanic membrane displacement with invasive intracranial pressure measurements. Physiol Meas 2005; 26(6): 1085-1092.

215. Kupersmith MJ, Sibony P, Mandel G, et al. Optical coherence tomography of the swollen optic nerve head: Deformation of the peripapillary retinal pigment epithelium layer in papilledema. Invest Ophthalmol Vis Sci 2011; 52(9): 6558-6564.

216. Popovich MJ, Hoffman WD. Noninvasive cardiac output monitoring. Crit Care Med 1997; 25(11): 1783-1784.

217. Selhorst JB, Gudeman SK, Butterworth JFt, et al. Papilledema after acute head injury. Neurosurgery 1985; 16(3): 357-363.

218. Vieira MA, Cavalcanti Mdo A, Costa DL, et al. Visual evoked potentials show strong positive association with intracranial pressure in patients with cryptococcal meningitis. Arq Neuropsiquiatr 2015; 73(4): 309-313.

219. Changa AR, Czeisler BM, Lord AS. Management of elevated intracranial pressure: A review. Curr Neurol Neurosci Rep 2019; 19(12): 99.

220. Rangel-Castilla L, Gopinath S, Robertson CS. Management of intracranial hypertension. Neurol Clin 2008; 26(2): 521-541.

221. Carney N, Totten AM, O'Reilly C, et al. Guidelines for the management of severe traumatic brain injury, fourth edition. Neurosurgery 2017; 80(1): 6-15.

222. Shevlin C. Optic nerve sheath ultrasound for the bedside diagnosis of intracranial hypertension: Pitfalls and potential. Critical Care Horizons 2015; 1(1): 22-30.

223. Hirzallah MI, Choi HAJJoNC. The monitoring of brain edema and intracranial hypertension. Journal of Neurocritical Care 2016; 9(2): 92-104.

224. Harary M, Dolmans RG, Gormley WBJS. Intracranial pressure monitoring-review and avenues for development. Sensors 2018; 18(2): 465.

225. Alali AS, Fowler RA, Mainprize TG, et al. Intracranial pressure monitoring in severe traumatic brain injury: Results from the american college of surgeons trauma quality improvement program. Journal of Neurotrauma 2013; 30(20): 1737-1746.

226. Farahvar A, Gerber LM, Chiu Y-L, et al. Increased mortality in patients with severe traumatic brain injury treated without intracranial pressure monitoring. Journal of Neurosurgery 2012; 117(4): 729-734.

227. Gerber LM, Chiu YL, Carney N, et al. Marked reduction in mortality in patients with severe traumatic brain injury. J Neurosurg 2013; 119(6): 1583-1590.

228. Talving $P$, Karamanos E, Teixeira PG, et al. Intracranial pressure monitoring in severe head injury: Compliance with brain trauma foundation guidelines and effect on outcomes: A prospective study. J Neurosurg 2013; 119(5): 1248-1254.

229. Chesnut RM, Temkin N, Carney N, et al. A trial of intracranial-pressure monitoring in traumatic brain injury. N Engl J Med 2012; 367(26): 2471-2481.

230. Cremer OL, van Dijk GW, van Wensen E, et al. Effect of intracranial pressure monitoring and targeted intensive care on functional outcome after severe head injury. Crit Care Med 2005; 33(10): 2207-2213.

231. Shafi S, Diaz-Arrastia R, Madden $C$, et al. Intracranial pressure monitoring in brain-injured patients is associated with worsening of survival. J Trauma 2008; 64(2): 335-340.

232. Powers WJ, Rabinstein AA, Ackerson T, et al. Guidelines for the early management of patients with acute ischemic stroke: 2019 update to the 2018 guidelines for the early management of acute ischemic stroke: A guideline for healthcare professionals from the american heart association/american stroke association. Stroke 2019; 50(12): e344-e418.

233. ESO Guidelines for management of ischaemic stroke and transient ischaemic attack 2008. Cerebrovasc Dis 2008; 25(5): 457-507.

234. Hernández-Durán S, Meinen L, Rohde V, et al. Invasive monitoring of intracranial pressure after decompressive craniectomy in malignant stroke. Stroke 2021; 52(2): 707-711.

235. Ferro JM, Crassard I, Coutinho JM, et al. Decompressive surgery in cerebrovenous thrombosis: A multicenter registry and a systematic review of individual patient data. Stroke 2011; 42(10): 2825-2831.

236. Roethlisberger M, Gut L, Zumofen DW, et al. Cerebral venous thrombosis requiring invasive treatment for elevated intracranial pressure in women with combined hormonal contraceptive intake: Risk factors, anatomical distribution, and clinical presentation. Neurosurg Focus 2018; 45(1): E12.

237. Godoy DA, Núñez-Patiño RA, Zorrilla-Vaca A, et al. Intracranial hypertension after spontaneous intracerebral hemorrhage: A systematic review and meta-analysis of prevalence and mortality rate. Neurocrit Care 2019; 31(1): 176-187.

238. Bratton SL, Chestnut RM, Ghajar J, et al. Guidelines for the management of severe traumatic brain injury. Viii. Intracranial pressure thresholds. J Neurotrauma 2007; 24 Suppl 1: S55-58.

239. Menacho ST, Grandhi R, Delic A, et al. Impact of intracranial pressure monitor-guided therapy on neurologic outcome after spontaneous nontraumatic intracranial hemorrhage. J Stroke Cerebrovasc Dis 2021; 30(3): 105540.

240. Jeon SB, Koh Y, Choi HA, et al. Critical care for patients with massive ischemic stroke. J Stroke 2014; 16(3): 146160.

241. Shah S, Kimberly WT. Today's approach to treating brain swelling in the neuro intensive care unit. Semin Neurol 2016; 36(6): 502-507.

242. Diringer MN. New trends in hyperosmolar therapy? Curr Opin Crit Care 2013; 19(2): 77-82.

243. Güngör L. Beyin ödemi ve tedavisi. Turkiye Klinikleri J Neurol-Special Topics 2015; 8(3): 26-30.

244. Mohney N, Alkhatib O, Koch S, et al. What is the role of hyperosmolar therapy in hemispheric stroke patients? Neurocrit Care 2020; 32(2): 609-619. 
245. Cook AM, Morgan Jones G, Hawryluk GWJ, et al. Guidelines for the acute treatment of cerebral edema in neurocritical care patients. Neurocrit Care 2020; 32(3): 647-666.

246. Nilius B, Droogmans G. Ion channels and their functional role in vascular endothelium. Physiol Rev 2001; 81(4): 1415-1459.

247. Dolman D, Drndarski S, Abbott NJ, et al. Induction of aquaporin 1 but not aquaporin 4 messenger rna in rat primary brain microvessel endothelial cells in culture. J Neurochem 2005; 93(4): 825-833.

248. Lam TI, Wise PM, O'Donnell ME. Cerebral microvascular endothelial cell na/h exchange: Evidence for the presence of nhe 1 and nhe 2 isoforms and regulation by arginine vasopressin. Am J Physiol Cell Physiol 2009; 297(2): C278-289.

249. Previch LE, Ma L, Wright JC, et al. Progress in aqp research and new developments in therapeutic approaches to ischemic and hemorrhagic stroke. Int J Mol Sci 2016; 17(7).

250. Yao $Y$, Zhang $Y$, Liao $X$, et al. Potential therapies for cerebral edema after ischemic stroke: A mini review. Front Aging Neurosci 2020; 12: 618819.

251. Mittal MK, LacKamp A. Intracerebral hemorrhage: Perihemorrhagic edema and secondary hematoma expansion: From bench work to ongoing controversies. Front Neurol 2016; 7: 210.

252. Zheng $\mathrm{H}$, Chen C, Zhang J, et al. Mechanism and therapy of brain edema after intracerebral hemorrhage. Cerebrovasc Dis 2016; 42(3-4): 155-169.

253. Wan YH, Nie C, Wang HL, et al. Therapeutic hypothermia (different depths, durations, and rewarming speeds) for acute ischemic stroke: A meta-analysis. J Stroke Cerebrovasc Dis 2014; 23(10): 2736-2747.

254. Powers WJ, Rabinstein AA, Ackerson T, et al. 2018 guidelines for the early management of patients with acute ischemic stroke: A guideline for healthcare professionals from the american heart association/american stroke association. Stroke 2018; 49(3): e46-e110.

255. Nawabi J, Flottmann F, Hanning U, et al. Futile recanalization with poor clinical outcome is associated with increased edema volume after ischemic stroke. Invest Radiol 2019; 54(5): 282-287.

256. Patel T, Zhou J, Piepmeier JM, et al. Polymeric nanoparticles for drug delivery to the central nervous system. Adv Drug Deliv Rev 2012; 64(7): 701-705.

257. Zhu S, Gao X, Huang K, et al. Glibenclamide enhances the therapeutic benefits of early hypothermia after severe stroke in rats. Aging Dis 2018; 9(4): 685-695.

258. Kunte H, Schmidt S, Eliasziw M, et al. Sulfonylureas improve outcome in patients with type 2 diabetes and acute ischemic stroke. Stroke 2007; 38(9): 2526-2530.

259. Silver FL, Fang J, Robertson AC, et al. Possible neuroprotective effects of sulforrylureas in diabetic patients with acute ischemic stroke. Stroke 2009; 40(4): E156.

260. Sheth KN, Kimberly WT, Elm JJ, et al. Exploratory analysis of glyburide as a novel therapy for preventing brain swelling. Neurocrit Care 2014; 21(1): 43-51.

261. Wang $\mathrm{X}$, Chang $\mathrm{Y}, \mathrm{He} \mathrm{Y}$, et al. Glimepiride and glibenclamide have comparable efficacy in treating acute ischemic stroke in mice. 2020; 162: 107845.

262. Pineda-Ramírez N, Alquisiras-Burgos I, Ortiz-Plata A, et al. Resveratrol activates neuronal autophagy through ampk in the ischemic brain. Molecular Neurobiology 2020; 57(2): 1055-1069.

263. Alquisiras-Burgos I, Ortiz-Plata A, Franco-Pérez J, et al. Resveratrol reduces cerebral edema through inhibition of de novo sur1 expression induced after focal ischemia. Exp Neurol 2020; 330: 113353.

264. Harrigan MR, Ennis SR, Sullivan SE, et al. Effects of intraventricular infusion of vascular endothelial growth factor on cerebral blood flow, edema, and infarct volume. Acta Neurochir (Wien) 2003; 145(1): 49-53.

265. Chi OZ, Hunter C, Liu X, et al. Effects of vegf and nitric oxide synthase inhibition on blood-brain barrier disruption in the ischemic and non-ischemic cerebral cortex. Neurol Res 2005; 27(8): 864-868.

266. Verkman AS, Smith AJ, Phuan PW, et al. The aquaporin-4 water channel as a potential drug target in neurological disorders. Expert Opin Ther Targets 2017; 21(12): 11611170.

267. Tang G, Yang GY. Aquaporin-4: A potential therapeutic target for cerebral edema. Int J Mol Sci 2016; 17(10).

268. Darabi S, Mohammadi MT. Fullerenol nanoparticles decrease ischaemia-induced brain injury and oedema through inhibition of oxidative damage and aquaporin-1 expression in ischaemic stroke. Brain Inj 2017; 31(8): $1142-1150$.

269. Yan Y, Dempsey RJ, Flemmer A, et al. Inhibition of na(+)$\mathrm{k}(+)-\mathrm{cl}(-)$ cotransporter during focal cerebral ischemia decreases edema and neuronal damage. Brain Res 2003; 961(1): 22-31.

270. Wang G, Huang $\mathrm{H}, \mathrm{He} \mathrm{Y}$, et al. Bumetanide protects focal cerebral ischemia-reperfusion injury in rat. Int J Clin Exp Pathol 2014; 7(4): 1487-1494.

271. $\mathrm{Xu} \mathrm{W}, \mathrm{Mu} \mathrm{X}$, Wang $\mathrm{H}$, et al. Chloride co-transporter nkcc1 inhibitor bumetanide enhances neurogenesis and behavioral recovery in rats after experimental stroke. Mol Neurobiol 2017; 54(4): 2406-2414.

272. Spasov AA, Murav'eva VU, Gurova NA, et al. Neuroprotective properties of a new inhibitor of na $+\mathrm{h}+$ exchanger (compound ru-1355) on the model of focal ischemia in rats. Eksp Klin Farmakol 2016; 79(4): 3-7.

273. Koyama Y, Matsui S, Itoh S, et al. The selective na+-ca2+ exchange inhibitor attenuates brain edema after radiofrequency lesion in rats. Eur J Pharmacol 2004; 489(3): 193-196.

274. Vakili A, Kataoka H, Plesnila N. Role of arginine vasopressin $\mathrm{v} 1$ and $\mathrm{v} 2$ receptors for brain damage after transient focal cerebral ischemia. J Cereb Blood Flow Metab 2005; 25(8): 1012-1019.

275. Ansari S, Krishnan R, Shahripour RB, et al. Combined antagonism of vasopressin receptor subtypes with conivaptan attenuates cerebral edema following ischemic stroke (p5. 202). : AAN Enterprises; 2018.

276. Zeynalov E, Jones SM, Elliott JP. Therapeutic time window for conivaptan treatment against stroke-evoked brain edema and blood-brain barrier disruption in mice. PLoS One 2017; 12(8): e0183985.

277. Cohen JA, Khatri B, Barkhof F, et al. Long-term (up to 4.5 years) treatment with fingolimod in multiple sclerosis: Results from the extension of the randomised transforms study. J Neurol Neurosurg Psychiatry 2016; 87(5): 468475.

278. Rolland WB, Lekic T, Krafft PR, et al. Fingolimod reduces cerebral lymphocyte infiltration in experimental models of rodent intracerebral hemorrhage. Exp Neurol 2013; 241: $45-55$. 
279. Wei Y, Yemisci M, Kim HH, et al. Fingolimod provides long-term protection in rodent models of cerebral ischemia. Ann Neurol 2011; 69(1): 119-129.

280. Talebi A, Rahnema M, Bigdeli MR. Effect of intravenous injection of antagomir-1 on brain ischemia. Mol Biol Rep 2019; 46(1): 1149-1155.

281. Zhang L, Xu S, Wu X, et al. Protective effects of the soluble epoxide hydrolase inhibitor 1-trifluoromethoxyphenyl-3(1-propionylpiperidin-4-yl) urea in a rat model of permanent middle cerebral artery occlusion. Front Pharmacol 2020; 11: 182.

282. Van der Worp H, Hofmeijer J, Jüttler E, et al. European stroke organisation (ESO) guidelines on the management of space-occupying brain infarction. European Stroke Journal 2021; 6(2): XC-CX.

283. Bevers MB, Kimberly WT. Critical care management of acute ischemic stroke. Curr Treat Options Cardiovasc Med 2017; 19(6): 41.

284. Farrokh S, Cho SM, Suarez JI. Fluids and hyperosmolar agents in neurocritical care: An update. Curr Opin Crit Care 2019; 25(2): 105-109.

285. Surani S, Lockwood G, Macias MY, et al. Hypertonic saline in elevated intracranial pressure: Past, present, and future. J Intensive Care Med 2015; 30(1): 8-12.

286. Hinson HE, Stein D, Sheth KN. Hypertonic saline and mannitol therapy in critical care neurology. J Intensive Care Med 2013; 28(1): 3-11.

287. Fink ME. Osmotherapy for intracranial hypertension: Mannitol versus hypertonic saline. Continuum (Minneap Minn) 2012; 18(3): 640-654.

288. García-Morales EJ, Cariappa R, Parvin CA, et al. Osmole gap in neurologic-neurosurgical intensive care unit: Its normal value, calculation, and relationship with mannitol serum concentrations. Crit Care Med 2004; 32(4): 986991.

289. Norris JW. Steroids may have a role in stroke therapy. Stroke 2004; 35(1): 228-229.

290. Sandercock PA, Soane T. Corticosteroids for acute ischaemic stroke. Cochrane Database Syst Rev 2011; 2011(9): Cd000064.

291. Steiner T, Juvela S, Unterberg A, et al. European stroke organization guidelines for the management of intracranial aneurysms and subarachnoid haemorrhage. Cerebrovasc Dis 2013; 35(2): 93-112.

292. Zaganas I, Halpin AP, Oleinik A, et al. A comparison of acute hemorrhagic stroke outcomes in 2 populations: The crete-boston study. Stroke 2011; 42(12): 3640-3642.

293. Feigin VL, Anderson N, Rinkel GJ, et al. Corticosteroids for aneurysmal subarachnoid haemorrhage and primary intracerebral haemorrhage. Cochrane Database Syst Rev 2005; (3): Cd004583.

294. Katayama Y, Haraoka J, Hirabayashi H, et al. A randomized controlled trial of hydrocortisone against hyponatremia in patients with aneurysmal subarachnoid hemorrhage. Stroke 2007; 38(8): 2373-2375.

295. Brouwer MC, McIntyre P, Prasad K, et al. Corticosteroids for acute bacterial meningitis. Cochrane Database Syst Rev 2015; 2015(9): Cd004405.

296. Prasad K, Singh MB, Ryan H. Corticosteroids for managing tuberculous meningitis. Cochrane Database Syst Rev 2016; 4(4): Cd002244.

297. Aguiar de Sousa D, Mestre T, Ferro JM. Cerebral venous thrombosis in behçet's disease: A systematic review. J Neurol 2011; 258(5): 719-727.

298. Chang SM, Messersmith H, Ahluwalia $M$, et al.
Anticonvulsant prophylaxis and steroid use in adults with metastatic brain tumors: Summary of sno and asco endorsement of the congress of neurological surgeons guidelines. Neuro Oncol 2019; 21(4): 424-427.

299. Czosnyka M, Brady K, Reinhard M, et al. Monitoring of cerebrovascular autoregulation: Facts, myths, and missing links. Neurocrit Care 2009; 10(3): 373-386.

300. Godoy DA, Seifi A, Garza D, et al. Hyperventilation therapy for control of posttraumatic intracranial hypertension. Front Neurol 2017; 8: 250.

301. Cold GE. Cerebral blood flow in acute head injury. The regulation of cerebral blood flow and metabolism during the acute phase of head injury, and its significance for therapy. Acta Neurochir Suppl (Wien) 1990; 49: 1-64.

302. Stocchetti N, Maas AI, Chieregato A, et al. Hyperventilation in head injury: A review. Chest 2005; 127(5): 1812-1827.

303. Curley G, Kavanagh BP, Laffey JG. Hypocapnia and the injured brain: More harm than benefit. Crit Care Med 2010; 38(5): 1348-1359.

304. Mayer SA, Chong JY. Critical care management of increased intracranial pressure. Journal of Intensive Care Medicine 2002; 17(2): 55-67.

305. Zhang Z, Guo Q, Wang E. Hyperventilation in neurological patients: From physiology to outcome evidence. Curr Opin Anaesthesiol 2019; 32(5): 568-573.

306. Coles JP, Minhas PS, Fryer TD, et al. Effect of hyperventilation on cerebral blood flow in traumatic head injury: Clinical relevance and monitoring correlates. Crit Care Med 2002; 30(9): 1950-1959.

307. Soustiel JF, Mahamid E, Chistyakov A, et al. Comparison of moderate hyperventilation and mannitol for control of intracranial pressure control in patients with severe traumatic brain injury - a study of cerebral blood flow and metabolism. Acta Neurochir (Wien) 2006; 148(8): 845-851.

308. Brandi G, Stocchetti N, Pagnamenta A, et al. Cerebral metabolism is not affected by moderate hyperventilation in patients with traumatic brain injury. Crit Care 2019; 23(1): 45.

309. Schizodimos T, Soulountsi V, Iasonidou C, et al. An overview of management of intracranial hypertension in the intensive care unit. J Anesth 2020; 34(5): 741-757.

310. Gelb AW, Craen RA, Rao GS, et al. Does hyperventilation improve operating condition during supratentorial craniotomy? A multicenter randomized crossover trial. Anesth Analg 2008; 106(2): 585-594, table of contents.

311. Bindu B, Bindra A, Rath G. Temperature management under general anesthesia: Compulsion or option. J Anaesthesiol Clin Pharmacol 2017; 33(3): 306-316.

312. Morrison SF, Nakamura K. Central mechanisms for thermoregulation. Annu Rev Physiol 2019; 81: 285-308.

313. González-Ibarra FP, Varon J, López-Meza EG. Therapeutic hypothermia: Critical review of the molecular mechanisms of action. Front Neurol 2011; 2: 4.

314. Polderman KH. Mechanisms of action, physiological effects, and complications of hypothermia. Crit Care Med 2009; 37(7 Suppl): S186-202.

315. Otto KA. Therapeutic hypothermia applicable to cardiac surgery. Vet Anaesth Analg 2015; 42(6): 559-569.

316. Wu TC, Grotta JC. Hypothermia for acute ischaemic stroke. Lancet Neurol 2013; 12(3): 275-284.

317. Kurisu K, Abumiya T, Nakamura H, et al. Transarterial regional brain hypothermia inhibits acute aquaporin-4 surge and sequential microvascular events in 
ischemia/reperfusion injury. Neurosurgery 2016; 79(1): 125-134.

318. Liu K, Khan $H$, Geng $X$, et al. Pharmacological hypothermia: A potential for future stroke therapy? Neurol Res 2016; 38(6): 478-490.

319. Rasmussen TP, Bullis TC, Girotra S. Targeted temperature management for treatment of cardiac arrest. Curr Treat Options Cardiovasc Med 2020; 22(11): 39.

320. Rittenberger JC, Doshi AA, Reynolds JC. Postcardiac arrest management. Emerg Med Clin North Am 2015; 33(3): 691-712.

321. Panchal AR, Bartos JA, Cabañas JG, et al. Part 3: Adult basic and advanced life support: 2020 american heart association guidelines for cardiopulmonary resuscitation and emergency cardiovascular care. Circulation 2020; 142(16_suppl_2): S366-s468.

322. Clark DL, Penner M, Wowk S, et al. Treatments (12 and $48 \mathrm{~h}$ ) with systemic and brain-selective hypothermia techniques after permanent focal cerebral ischemia in rat. Exp Neurol 2009; 220(2): 391-399.

323. Kuczynski AM, Marzoughi S, Al Sultan AS, et al. Therapeutic hypothermia in acute ischemic stroke-a systematic review and meta-analysis. Curr Neurol Neurosci Rep 2020; 20(5): 13.

324. Yao Z, You C, He M. Effect and feasibility of therapeutic hypothermia in patients with hemorrhagic stroke: A systematic review and meta-analysis. World Neurosurg 2018; 111: 404-412.e402.

325. Polderman $\mathrm{KH}$, editor Is therapeutic hypothermia immunosuppressive? Critical Care; 2012; BioMed Central.

326. Zydlewski AW, Hasbargen JA. Hypothermia-induced hypokalemia. Mil Med 1998; 163(10): 719-721.

327. Luscombe M, Andrzejowski JC. Clinical applications of induced hypothermia. Continuing Education in Anaesthesia Critical Care \& Pain 2006; 6(1): 23-27.

328. Khan JN, Prasad N, Glancy JM. Qtc prolongation during therapeutic hypothermia: Are we giving it the attention it deserves? Europace 2010; 12(2): 266-270.

329. Timofeev I, Dahyot-Fizelier C, Keong N, et al. Ventriculostomy for control of raised icp in acute traumatic brain injury. Acta Neurochir Suppl 2008; 102: 99-104.

330. Tuncer M. Beyin ve sinir cerrahisinde kanita dayalı rehberler. Ankara: Buluș Tasarım ve Matbaacılık Hizmetleri; 2020; 286-291.

331. Dossani RH, Patra DP, Terrell DL, et al. Placement of an external ventricular drain. N Engl J Med 2021; 384(2): e3.

332. Bennett TD, Riva-Cambrin J, Keenan HT, et al. Variation in intracranial pressure monitoring and outcomes in pediatric traumatic brain injury. Arch Pediatr Adolesc Med 2012; 166(7): 641-647.

333. Bales JW, Bonow RH, Buckley RT, et al. Primary external ventricular drainage catheter versus intraparenchymal icp monitoring: Outcome analysis. Neurocrit Care 2019; 31(1): 11-21.

334. Hepburn-Smith M, Dynkevich I, Spektor M, et al. Establishment of an external ventricular drain best practice guideline: The quest for a comprehensive, universal standard for external ventricular drain care. J Neurosci Nurs 2016; 48(1): 54-65.

335. Dey M, Stadnik A, Riad F, et al. Bleeding and infection with external ventricular drainage: A systematic review in comparison with adjudicated adverse events in the ongoing clot lysis evaluating accelerated resolution of intraventricular hemorrhage phase III trial. Neurosurgery 2015; 76(3): 291-300.

336. Bauer DF, McGwin G, Jr., Melton SM, et al. The relationship between inr and development of hemorrhage with placement of ventriculostomy. J Trauma 2011; 70(5): 1112-1117.

337. Binz DD, Toussaint LG, 3rd, Friedman JA. Hemorrhagic complications of ventriculostomy placement: A metaanalysis. Neurocrit Care 2009; 10(2): 253-256.

338. Fried HI, Nathan BR, Rowe AS, et al. The insertion and management of external ventricular drains: An evidencebased consensus statement : A statement for healthcare professionals from the neurocritical care society. Neurocrit Care 2016; 24(1): 61-81.

339. Zabramski JM, Whiting D, Darouiche RO, et al. Efficacy of antimicrobial-impregnated external ventricular drain catheters: A prospective, randomized, controlled trial. J Neurosurg 2003; 98(4): 725-730.

340. Slazinski T, Anderson T, Cattell E, et al. Care of the patient undergoing intracranial pressure monitoring/external ventricular drainage or lumbar drainage. Glenview I, editor: American Association of Neuroscience Nurses; 2011; 1-38.

341. Dellit TH, Chan JD, Fulton C, et al. Reduction in clostridium difficile infections among neurosurgical patients associated with discontinuation of antimicrobial prophylaxis for the duration of external ventricular drain placement. Infect Control Hosp Epidemiol 2014; 35(5): 589-590.

342. $\mathrm{Gu} \mathrm{JC}, \mathrm{Wu} \mathrm{H}, \mathrm{Chen} \mathrm{XZ}$, et al. Intracranial pressure during external ventricular drainage weaning is an outcome predictor of traumatic brain injury. Biomed Res Int 2020; 2020: 8379134 .

343. Jungheinrich $C$, Scharpf $R$, Wargenau $M$, et al. The pharmacokinetics and tolerability of an intravenous infusion of the new hydroxyethyl starch 130/0.4 (6\%, $500 \mathrm{ml}$ ) in mild-to-severe renal impairment. Anesth Analg 2002; 95(3): 544-551, table of contents.

344. Walder B, Tramèr MR, Seeck M. Seizure-like phenomena and propofol: A systematic review. Neurology 2002; 58(9): 1327-1332.

345. Bryson HM, Fulton BR, Faulds D. Propofol. An update of its use in anaesthesia and conscious sedation. Drugs 1995; 50(3): 513-559.

346. Severe Medicine Branch of Chinese Medical Association. Chinese guidelines for analgesia and sedation in icu for adults. Chinese Crit Emerge Med 2018; 30(6): 49.

347. Dapeng R, Wenjuan C, Wenqiang L, et al. Effects of propofol combined with dexmedetomidine on circulatory system and sedation in patients with mechanical ventilation in intensive care unit. Trauma Crit Ilness Med 2018; 11(6): 372-374.

348. Oddo M, Steiner LA. Sedation and analgesia in the neurocritical care unit. Oxford textbook of neurocritical care: Oxford University Press; 2016.

349. Ziai W, Sajjad R. Analgesia, sedation and paralysis. In: Torbey M, editor. Neurocrit care: Cambridge Univesity Press; 2009.

350. Mirrakhimov AE, Voore P, Halytskyy O, et al. Propofol infusion syndrome in adults: A clinical update. Crit Care Res Pract 2015; 2015: 260385.

351. Robba C, Citerio G. How i manage intracranial hypertension: BioMed Central; 2019.

352. Flower O, Hellings S. Sedation in traumatic brain injury. Emergency medicine international 2012; 2012. 
353. Mishra LD. Cerebral blood flow and anaesthesia: A review. Indian J Anaesth 2002; 46(2): 87-95.

354. Öge E, Baykan B. Nöroloji. 2. Baskı. İstanbul: Nobel Tıp Kitabevi; 2011; 218.

355. Pratt 0 , Bowles B, Protheroe R. Brain stem death testing after thiopental use: A survey of uk neuro critical care practice. Anaesthesia 2006; 61(11): 1075-1078.

356. Wijdicks EF, Sheth KN, Carter BS, et al. Recommendations for the management of cerebral and cerebellar infarction with swelling: A statement for healthcare professionals from the american heart association/american stroke association. Stroke 2014; 45(4): 1222-1238.

357. Kocher T. Hirnerschütterung, hirndruck und chirurgische eingriffe bei hirnkrankheiten: A. Hölder; 1901.

358. Cushing $\mathrm{H}$. The establishment of cerebral hernia as a decompressive measure for inaccessible brain tumors: With the description of intermuscular methods of making the bone defect in temporal and occipital regions. Surg Gynecol Obstet 1905; 1: 297-314.

359. Ivamoto HS, Numoto $M$, Donaghy RM. Surgical decompression for cerebral and cerebellar infarcts. Stroke 1974; 5(3): 365-370.

360. Kjellberg RN, Prieto A, Jr. Bifrontal decompressive craniotomy for massive cerebral edema. J Neurosurg 1971; 34(4): 488-493.

361. Timofeev I, Santarius T, Kolias A, et al. Decompressive craniectomy-operative technique and perioperative care. Advances and technical standards in neurosurgery: Springer; 2012; 115-136.

362. Kunt R, Yaka E, Öztürk V, ve ark. İnmed dekompresif cerrahi. Türk Beyin Damar Hastalıkları Dergisi 2014; 20(1): 13-17.

363. Carney N, Totten AM, O'Reilly C, et al. Guidelines for the management of severe traumatic brain injury. Neurosurgery 2017; 80(1): 6-15.

364. Wagner S, Schnippering H, Aschoff A, et al. Suboptimum hemicraniectomy as a cause of additional cerebral lesions in patients with malignant infarction of the middle cerebral artery. Journal of neurosurgery 2001; 94(5): 693-696.

365. Corliss B, Gooldy T, Vaziri S, et al. Complications after in vivo and ex vivo autologous bone flap storage for cranioplasty: A comparative analysis of the literature. World neurosurgery 2016; 96: 510-515.

366. Schuss $P$, Vatter H, Marquardt G, et al. Cranioplasty after decompressive craniectomy: The effect of timing on postoperative complications. Journal of neurotrauma 2012; 29(6): 1090-1095.

367. İlgezdi İ, Öcek L, Binyay LA, et al. Decompressive hemicraniectomy in acute ischemic stroke Turkish Journal of Cerebrovascular Diseases 2019; 25(1): 31-36.

368. Gopalakrishnan M, Shanbhag NC, Shukla DP, et al. Complications of decompressive craniectomy. Frontiers in neurology 2018; 9: 977.

369. Schirmer CM, Ackil AA, Malek AM. Decompressive craniectomy. Neurocritical care 2008; 8(3): 456-470.

370. Creutzfeldt C, Tirschwell D, Kim L, et al. Seizures after decompressive hemicraniectomy for ischaemic stroke. Journal of Neurology, Neurosurgery \& Psychiatry 2014; 85(7): 721-725.

371. Kurland DB, Khaladj-Ghom A, Stokum JA, et al. Complications associated with decompressive craniectomy: A systematic review. Neurocritical care 2015; 23(2): 292-304.

372. Aarabi B, Hesdorffer DC, Simard JM, et al. Comparative study of decompressive craniectomy after mass lesion evacuation in severe head injury. Neurosurgery 2009; 64(5): 927-940.

373. Vahedi K, Vicaut E, Mateo J, et al. Sequential-design, multicenter, randomized, controlled trial of early decompressive craniectomy in malignant middle cerebral artery infarction (decimal trial). Stroke 2007; 38(9): 2506-2517.

374. Jüttler E, Schwab S, Schmiedek P, et al. Decompressive surgery for the treatment of malignant infarction of the middle cerebral artery (destiny) a randomized, controlled trial. Stroke 2007; 38(9): 2518-2525.

375. Hofmeijer J, Kappelle LJ, Algra A, et al. Surgical decompression for space-occupying cerebral infarction (the hemicraniectomy after middle cerebral artery infarction with life-threatening edema trial [hamlet]): A multicentre, open, randomised trial. The Lancet Neurology 2009; 8(4): 326-333.

376. Slezins J, Keris V, Bricis R, et al. Preliminary results of randomized controlled study on decompressive craniectomy in treatment of malignant middle cerebral artery stroke. Medicina 2012; 48(10): 76.

377. Zhao J, Su YY, Zhang Y, et al. Decompressive hemicraniectomy in malignant middle cerebral artery infarct: A randomized controlled trial enrolling patients up to 80 years old. Neurocritical care 2012; 17(2): 161171.

378. Frank JI, Schumm LP, Wroblewski K, et al. Hemicraniectomy and durotomy upon deterioration from infarction-related swelling trial: Randomized pilot clinical trial. Stroke 2014; 45(3): 781-787.

379. Jüttler E, Unterberg A, Woitzik J, et al. Hemicraniectomy in older patients with extensive middle-cerebral-artery stroke. New England Journal of Medicine 2014; 370(12): 1091-1100.

380. Chua AE, Buckley BS, Lapitan M, et al. Hemicraniectomy for malignant middle cerebral artery infarction (hemmi): A randomized controlled clinical trial of decompressive surgery with standardized medical care versus standardized medical care alone. Acta Med Philipp 2015; 49(1): 28-33.

381. Vahedi K, Hofmeijer J, Juettler E, et al. Early decompressive surgery in malignant infarction of the middle cerebral artery: A pooled analysis of three randomised controlled trials. The Lancet Neurology 2007; 6(3): 215-222.

382. Cruz-Flores S, Berge E, Whittle IR. Surgical decompression for cerebral oedema in acute ischaemic stroke. Cochrane Database of Systematic Reviews 2012; (1).

383. Back L, Nagaraja V, Kapur A, et al. Role of decompressive hemicraniectomy in extensive middle cerebral artery strokes: A meta-analysis of randomised trials. Internal medicine journal 2015; 45(7): 711-717.

384. Alexander P, Heels-Ansdell D, Siemieniuk R, et al. Hemicraniectomy versus medical treatment with large mca infarct: A review and meta-analysis. BMJ open 2016; 6(11): e014390.

385. Das S, Mitchell $\mathrm{P}$, Ross $\mathrm{N}$, et al. Decompressive hemicraniectomy in the treatment of malignant middle cerebral artery infarction: A meta-analysis. World neurosurgery 2019; 123: 8-16.

386. Reinink $H$, Jüttler $E$, Hacke $W$, et al. Surgical decompression for space-occupying hemispheric infarction: A systematic review and individual patient 
meta-analysis of randomized clinical trials. JAMA Neurol. 2021;78(2):208-216.

387. Ongun N, Marangoz D, Değirmenci E, ve ark. Geniş orta serebral arter enfarktında dekompresif cerrahi ve yalnızca medikal tedavi sonuçlarının karşılaştırılması. Pamukkale Tip Dergisi ; 10(2): 136-142.

388. Gupta R, Connolly ES, Mayer S, et al. Hemicraniectomy for massive middle cerebral artery territory infarction: A systematic review. Stroke 2004; 35(2): 539-543.

389. Dasenbrock HH, Robertson FC, Vaitkevicius $\mathrm{H}$, et al. Timing of decompressive hemicraniectomy for stroke: A nationwide inpatient sample analysis. Stroke 2017; 48(3): 704-711.

390. Caplan LR. Cerebellar infarcts: Key features. Rev Neurol Dis 2005; 2(2): 51-60.

391. Taylor DR, Basma J, Jones GM, et al. Predicting surgical intervention in cerebellar stroke: A quantitative retrospective analysis. World Neurosurgery 2020; 142: e160-e172.

392. Tchopev Z, Hiller M, Zhuo J, et al. Prediction of poor outcome in cerebellar infarction by diffusion mri. Neurocritical care 2013; 19(3): 276-282.

393. Powers WI, Rabinstein AA, Ackerson T, et al. Guidelines for the early management of patients with acute ischemic stroke: 2019 update to the 2018 guidelines for the early management of acute ischemic stroke: A guideline for healthcare professionals from the american heart association/american stroke association. Stroke 2019; 50(12): e344-e418.

394. Jauss M, Krieger D, Hornig C, et al. Surgical and medical management of patients with massive cerebellar infarctions: Results of the German-Austrian cerebellar infarction study. Journal of neurology 1999; 246(4): 257264.

395. Pallesen L-P, Barlinn K, Puetz V. Role of decompressive craniectomy in ischemic stroke. Frontiers in neurology 2019; 9: 1119.

396. Pfefferkorn T, Eppinger U, Linn J, et al. Long-term outcome after suboccipital decompressive craniectomy for malignant cerebellar infarction. Stroke 2009; 40(9): 3045-3050.

397. Kim MJ, Park SK, Song J, et al. Preventive suboccipital decompressive craniectomy for cerebellar infarction: A retrospective-matched case-control study. Stroke 2016; 47(10): 2565-2573.

398. Beez T, Munoz-Bendix C, Steiger H-J, et al. Decompressive craniectomy for acute ischemic stroke. Critical Care 2019; 23(1): 1-16.

399. Murthy J, Chowdary G, Murthy T, et al. Decompressive craniectomy with clot evacuation in large hemispheric hypertensive intracerebral hemorrhage. Neurocritical care 2005; 2(3): 258-262.

400. Heuts SG, Bruce SS, Zacharia BE, et al. Decompressive hemicraniectomy without clot evacuation in dominantsided intracerebral hemorrhage with icp crisis. Neurosurgical focus 2013; 34(5): E4.

401. Esquenazi Y, Savitz SI, El Khoury R, et al. Decompressive hemicraniectomy with or without clot evacuation for large spontaneous supratentorial intracerebral hemorrhages. Clinical neurology and neurosurgery 2015; 128: 117-122.

402. Lo YT, See AAQ, King NKK. Decompressive craniectomy in spontaneous intracerebral hemorrhage: A case-control study. World Neurosurgery 2017; 103: 815-820. e812.
403. de Oliveira Manoel AL. Surgery for spontaneous intracerebral hemorrhage. Critical Care 2020; 24(1): 45.

404. Gildersleeve KL, Hirzallah MI, Esquenazi $\mathrm{Y}$, et al. Hemicraniectomy for supratentorial primary intracerebral hemorrhage: A retrospective, propensity score matched study. Journal of Stroke and Cerebrovascular Diseases 2019; 28(11): 104361.

405. Mendelow AD, Gregson BA, Fernandes HM, et al. Early surgery versus initial conservative treatment in patients with spontaneous supratentorial intracerebral haematomas in the international surgical trial in intracerebral haemorrhage (stich): A randomised trial. The Lancet 2005; 365(9457): 387-397.

406. Mendelow AD, Gregson BA, Rowan EN, et al. Early surgery versus initial conservative treatment in patients with spontaneous supratentorial lobar intracerebral haematomas (stich ii): A randomised trial. The Lancet 2013; 382(9890): 397-408.

407. Steiner T, Salman RA-S, Beer R, et al. European stroke organisation (ESO) guidelines for the management of spontaneous intracerebral hemorrhage. International journal of stroke 2014; 9(7): 840-855.

408. Takeuchi S, Wada K, Nagatani K, et al. Decompressive hemicraniectomy for spontaneous intracerebral hemorrhage. Neurosurgical focus 2013; 34(5): E5.

409. Luney M, English S, Longworth A, et al. Acute posterior cranial fossa hemorrhage-is surgical decompression better than expectant medical management? Neurocritical care 2016; 25(3): 365-370.

410. Hemphill III JC, Greenberg SM, Anderson CS, et al. Guidelines for the management of spontaneous intracerebral hemorrhage: A guideline for healthcare professionals from the american heart association/american stroke association. Stroke 2015; 46(7): 2032-2060.

411. Çiftçi Ș, Güler A, Șirin H, ve ark. Malin sinüs ven trombozu ve dekompresif kraniektomi. Türk Beyin Damar Hastalıkları Dergisi 2016; 22(3): 107-109.

\section{Ethics}

Ethics Committee Approval: This is a review article and there is no need an Ethical Aprrovel.

Copyright Transfer Form: All authors signed the Copyright Transfer Form.

Peer-review: Internally peer-reviewed.

Authorship Contributions: Concept: LG, HȘ, Design: LG, HȘ, Literature Search: All authors, Writing: The article chapters were brought together, edited and redacted by Levent Güngör and Hadiye Şirin. The authors of the subsections are as follows: 1.0 Introduction (Levent Güngör, Hadiye Şirin), 1.1 Brain edema, raised ICP syndrome and herniation in stroke: Definitions and epidemiology: (Canan Togay Işıkay), 1.2 Effect of brain edema on prognosis in acute ischemic stroke (Mustafa Gökçe), 1.3. The effect of brain edema on prognosis in intracerebral hemorrhages (Ayça Özkul, Anıl Tanburoğlu), 2.1 Overview and pathophysiology of raised intracranial pressure syndrome (Nazire Afşar, Levent Güngör), 2.2 Brain edema types and physiopathology in acute ischemic stroke (Arda Yılmaz, Canan Togay Ișlkay, Nazire Afșar), 2.2.1 The bloodbrain barrier and the neurovascular unit (Müge Yemișci, Gökçe Gürler), 2.2.2. Inter-endothelial tight junctions (Yasemin Görsoy Özdemir), 2.2.3 NKCC1 and cerebral edema (Bijen Nazlıel, Mine Hayriye Sorgun), 2.2.4. Sur1-Trpm4 channels and cerebral edema (Murat Polat, Nilda Turgut, Levent Güngör), 
Güngör et al.

2.2.5 The role of aquaporin channels in cerebral edema (Baki Doğan, Erdem Gürkaș, Levent Güngör), 2.2.6 Different aspects of reperfusion edema (Ufuk Can, Özlem Aykaç), 2.3 Perihematomal brain edema in intracerebral hemorrhages (Zülfikar Arlıer, Ülgen Yalaz Tekan), 3.1 Herniation syndromes in stroke: Types and clinical findings (Kürşad Kutluk, Vedat Ali Yürekli), 3.2 Herniation syndromes in ischemia: CT findings (Bilgehan Atılgan Acar, Erdem Yaka), 3.3 Radiological diagnosis of brain edema types (Dilaver Kaya), 3.4.1. Transcranial Doppler sonography (Ali Ünal), 3.4.2. Transcranial B-mode sonography (Mehmet Akif Topçuoğlu), 3.4.3 Optic nerve sheath diameter measurement (Ayșe Güler, Hale Zeynep Batur Çağlayan), 3.5 Pupillometry and other non-invasive methods in the diagnosis of rICP (Mehmet Yasir Pektezel, Dilek Necioğlu Örken), 3.6. Invasive monitoring of intracranial pressure (Hasan Hüseyin Kozak, Tuğçe Mengi), 4. treatment of cerebral edema in stroke, 4.1 General principles (Levent Güngör), 4.2. Treatment of cytotoxic cerebral edema (Şebnem Bıçakçı, Turgay Demir), 4.3 Treatment of ionic edema (Yasemin Dinç, Mustafa Bakar), 4.4 Treatment of vasogenic edema (Nilüfer Yeşilot, Fatma Birsen İnce), 4.5 Future treatments for cerebral edema (Özlem Kayım Yıldız, Recep Baydemir), 5.1 Osmotherapy (Belgin Koçer, Hadiye Şirin), 5.2 Osmometry in osmotherapy monitoring (Ethem Murat Arsava), 5.3 The role of corticosteroids in the treatment of cerebral edema (Burcu Acar Çinleti, Şerefnur Öztürk), 5.4 KIBAS tedavisinde hiperventilasyon (Vesile Öztürk, Ezgi Sezer Eryıldız), 5.5 KIBAS tedavisinde terapötik hipotermi ve hedeflenmiş sıcaklık yönetimi (Derya Tatlısuluoğlu, Aysel Milanlığlu), 5.6 KIBAS tedavisinde eksternal ventriküler drenaj (Babür Dora, Hamza Gültekin), 5.7 KiBAS'ta sedasyon uygulamaları, 5.7.1 Propofol (Ayșe Bingöl, Nazan Duman), 5.7.2. Pentotal koması (Mehmet Uğur Çevik, Zehra Uysal Kocabaş), 6.1 Decompressive surgery in malignant middle cerebral artery infarction (Atilla Özcan Özdemir, Zehra Uysal), 6.2 İ Posterior decompression in ischemic stroke (Semih Giray, Demet Funda Baş), 6.3 Decompressive craniectomy in intracerebral hemorrhages (İpek Midi), 7.0 Afterword (Hadiye Şirin, Levent Güngör).

Conflict of Interest: No conflict of interest was declared by the authors.

Financial Disclosure: The authors declared that this study received no financial support. 